\title{
Chemoreceptor Co-Expression in Drosophila Olfactory Neurons
}

Darya Task ${ }^{1,10}$, Chun-Chieh Lin ${ }^{1,2,7}$, Alina Vulpe ${ }^{3}$, Ali Afify ${ }^{1}$, Sydney Ballou ${ }^{3}$, Maria Brbić ${ }^{4}$, Philipp Schlege $\left.\right|^{5,8}$, Gregory S. X. E. Jefferis ${ }^{5,8}$, Hongjie Lij ${ }^{6,9}$, Karen Menuz ${ }^{3}$ and Christopher J. Potter ${ }^{1 *}$ (1)

${ }^{1}$ The Solomon H. Snyder Department of Neuroscience, Center for Sensory Biology, Johns Hopkins University School of Medicine, Baltimore, MD 21205, USA.

${ }^{2}$ Mortimer B. Zuckermann Mind Brain Behavior Institute, Columbia University, New York, NY 10027, USA.

${ }^{3}$ Physiology \& Neurobiology Department, University of Connecticut, Mansfield, CT 06269, USA. ${ }^{4}$ Department of Computer Science, Stanford University, Stanford, CA 94305, USA.

${ }^{5}$ Drosophila Connectomics Group, Department of Zoology, University of Cambridge, Cambridge, CB2 3EJ, UK.

${ }^{6}$ Department of Biology, Howard Hughes Medical Institute, Stanford University, Stanford, CA 94305, USA.

${ }^{7}$ Present Address: The Department of Pathology and Laboratory Medicine, Giesel School of Medicine, Dartmouth-Hitchcock Medical Center, Lebanon, NH 03756, USA.

${ }^{8}$ Neurobiology Division, MRC Laboratory of Molecular Biology, Cambridge, CB2 OQH, UK. ${ }^{9}$ Present Address: Huffington Center on Aging, Department of Molecular and Human Genetics, Baylor College of Medicine, Houston, TX 77030, USA.

${ }^{10}$ Present Address: Department of Biology, Johns Hopkins University, Baltimore, MD 21218 , USA.

*Correspondence: cpotter@jhmi.edu 


\section{Abstract}

24 Drosophila melanogaster olfactory neurons have long been thought to express only one chemosensory receptor gene family. There are two main olfactory receptor gene families in Drosophila, the Odorant Receptors (ORs) and the lonotropic Receptors (IRs). The dozens of odorant binding receptors in each family require at least one co-receptor gene in order to function: Orco for ORs, and Ir25a, Ir8a, and Ir76b for IRs. Using a new genetic knock-in strategy, we targeted the four co-receptors representing the main chemosensory families in Drosophila (Orco, Ir8a, Ir76b, Ir25a). Co-receptor knock-in expression patterns were verified as accurate representations of endogenous expression. We find extensive overlap in expression among the different co-receptors. As defined by innervation into antennal lobe glomeruli, Ir25a is broadly expressed in $88 \%$ of all olfactory sensory neuron classes and is co-expressed in $82 \%$ of Orco+ neuron classes, including all neuron classes in the maxillary palp. Orco, Ir8a, and Ir76b expression patterns are also more expansive than previously assumed. Single sensillum recordings from Orco-expressing Ir25a mutant antennal and palpal neurons identify changes in olfactory responses. These results suggest co-expression of chemosensory receptors is common in olfactory neurons. Together, our data present the first comprehensive map of chemosensory co-receptor expression and reveal their unexpected widespread co-expression in the fly olfactory system.

\section{Keywords:}

42 Insect, olfaction, Odorant Receptor, lonotropic Receptor, sensory neuron, CRISPR, QF2, 43 antennal lobe, HACK, knock-in.

\section{Introduction}

45 The sense of smell is crucial for many animal behaviors, from conspecific recognition and mate 46 choice (Dweck et al., 2015; Stengl, 2010), to location of a food source (Auer et al., 2020; 
47 Hansson and Stensmyr, 2011), to avoidance of predators (Kondoh et al., 2016; Papes et al., 2010) and environmental dangers (Mansourian et al., 2016; Stensmyr et al., 2012). Peripheral sensory organs detect odors in the environment using a variety of chemosensory receptors (Carey and Carlson, 2011; Su et al., 2009). The molecular repertoire of chemosensory receptors expressed by the animal, and the particular receptor expressed by any individual olfactory neuron, define the rules by which an animal interfaces with its odor environment.

53 Investigating this initial step in odor detection is critical to understanding how odor signals first enter the brain to guide behaviors. extensively studied and well understood (Depetris-Chauvin et al., 2015). Drosophila is an attractive model for studying olfaction due to its genetic tractability, numerically simpler nervous system (compared to mammals), complex olfactory-driven behaviors, and similar organizational principles to vertebrate olfactory systems (Ache and Young, 2005; Wilson, 2013). Over sixty years of research have elucidated many of the anatomical, molecular, and genetic principles underpinning fly olfactory behaviors (Gomez-Diaz et al., 2018; Harris, 1972; Pask and Ray, 2016; Siddiqi, 1987; Stocker, 2001; Venkatesh and Singh, 1984; Vosshall and Stocker, 2007; Yan et al., 2020). Recent advances in electron microscopy and connectomics are revealing outputs. higher brain circuits involved in processing of olfactory information (Bates et al., 2020; Berck et al., 2016; Frechter et al., 2019; Horne et al., 2018; Marin et al., 2020; Zheng et al., 2018); such endeavors will aid the full mapping of neuronal circuits from sensory inputs to behavioral

The fly uses two olfactory appendages to detect odorants: the antennae and maxillary palps (Figure 1A) (Stocker, 1994). Each of these is covered by sensory hairs called sensilla, and each sensillum houses between one and four olfactory sensory neurons or OSNs (Figure

71 1B) (de Bruyne et al., 2001; Venkatesh and Singh, 1984). The dendrites of these neurons are

72 found within the sensillar lymph, and they express chemosensory receptors from three gene 
families: Odorant Receptors (ORs), Ionotropic Receptors (IRs), and Gustatory Receptors (GRs) (Figure 1C, left) (Benton et al., 2009; Clyne et al., 1999; Gao and Chess, 1999; Jones et al., 2007; Kwon et al., 2007; Vosshall et al., 1999; Vosshall et al., 2000). These receptors bind odorant molecules that enter the sensilla from the environment, leading to the activation of the OSNs, which then send this olfactory information to the fly brain (Figure 1D), to the first olfactory processing center - the antennal lobes (ALs) (Figure 1E) (reviewed in: Depetris-Chauvin et al., 2015; Gomez-Diaz et al., 2018; Pask and Ray, 2016). The standard view regarding the organization of the olfactory system in Drosophila is that olfactory neurons express receptors from only one of the chemosensory gene families (either ORs, IRs, or GRs), and all neurons expressing the same receptor (which can be considered an OSN class) project their axons to one specific region in the $\mathrm{AL}$ called a glomerulus (Figure $1 \mathrm{C}$, right) (Couto et al., 2005;

Fishilevich and Vosshall, 2005; Gao et al., 2000; Laissue et al., 1999; Pinto et al., 1988;

Vosshall et al., 2000). This pattern of projections creates a map in which the OR+ (Figure 1E, teal), IR+ (Figure 1E, purple), and GR+ (Figure 1E, dark blue) domains are segregated from each other in the AL. The OR+ domains innervate 38 anterior glomeruli, while the IR+ (19 glomeruli) and GR+ (1 glomerulus) domains occupy more posterior portions of the AL. One exception is the Or35a+ OSN class, which expresses an $I R(I r 76 b)$ in addition to the $O R$ and Orco, and innervates the VC3 glomerulus (Figure 1E, striped glomerulus) (Benton et al., 2009; Couto et al., 2005; Fishilevich and Vosshall, 2005). Different OSN classes send their information to different glomeruli, and the specific combination of OSN classes and glomeruli that are activated by a given smell (usually a blend of different odorants) constitutes an olfactory 'code' which the fly brain translates into an appropriate behavior (Grabe and Sachse, 2018; Haverkamp et al., 2018; Seki et al., 2017).

The receptors within each chemosensory gene family form heteromeric ion channels (receptor complexes) (Abuin et al., 2011; Butterwick et al., 2018; Sato et al., 2008). The ORs require a single co-receptor, Orco, to function (Figure 1C, middle row) (Benton et al., 2006; 
Larsson et al., 2004; Vosshall and Hansson, 2011). The ligand-binding OrX confers odorant specificity upon the receptor complex, while the co-receptor Orco is necessary for trafficking of the OrX to the dendritic membrane and formation of a functional ion channel (Benton et al., 2006; Larsson et al., 2004). Likewise, the ligand-binding IrXs require one or more IR coreceptors: Ir8a, Ir25a, and/or Ir76b (Figure 1C, top row). The IR co-receptors are similarly required for trafficking and ion channel function (Abuin et al., 2011; Abuin et al., 2019; Ai et al., 105 2013; Vulpe et al., 2021b). The GR gene family generally encodes receptors involved in taste, which are typically expressed outside the olfactory system (such as in the labella or the legs)

107 (Dunipace et al., 2001; Park and Kwon, 2011; Scott, 2018; Scott et al., 2001); however, Gr21a and Gr63a are expressed in one antennal OSN neuron class, and form a complex sensitive to carbon dioxide (Figure 1C, bottom row) (Jones et al., 2007; Kwon et al., 2007).

The majority of receptors have been mapped to their corresponding OSNs, sensilla, and

111 glomeruli in the fly brain (Bhalerao et al., 2003; Couto et al., 2005; Fishilevich and Vosshall,

112 2005; Frank et al., 2017; Grabe et al., 2016; Hallem and Carlson, 2006; Hallem et al., 2004;

113 Knecht et al., 2017; Marin et al., 2020; Ray et al., 2008; Silbering et al., 2011). This detailed

114 map has allowed for exquisite investigations into the developmental, molecular,

115 electrophysiological, and circuit/computational bases of olfactory neurobiology. This work has

116 relied on transgenic lines to identify and manipulate OSN classes (Ai et al., 2013; Brand and

117 Perrimon, 1993; Couto et al., 2005; Fishilevich and Vosshall, 2005; Kwon et al., 2007; Lai and

118 Lee, 2006; Larsson et al., 2004; Menuz et al., 2014; Potter et al., 2010; Silbering et al., 2011).

119 These transgenic lines use segments of DNA upstream of the chemosensory genes that are 120 assumed to reflect the enhancers and promoters driving expression of these genes. While a 121 powerful tool, transgenic lines may not contain all of the necessary regulatory elements to 122 faithfully recapitulate the expression patterns of the endogenous genes. Some transgenic lines 123 label a subset of the cells of a given olfactory class, while others label additional cells: for 124 example, the transgenic Ir25a-Gal4 line is known to label only a portion of cells expressing Ir25a 
125

126

127

128

129

protein (as revealed by antibody staining) (Abuin et al., 2011); conversely, Or67d-Gal4 transgenes incorrectly label two glomeruli, whereas a Gal4 knock-in at the Or67d genetic locus labels a single glomerulus (Couto et al., 2005; Fishilevich and Vosshall, 2005; Kurtovic et al., 2007). While knock-ins provide a faithful method to capture a gene's expression pattern, generating these lines has traditionally been cumbersome.

In this paper, we implement an efficient knock-in strategy to target the four main chemosensory co-receptor genes in Drosophila melanogaster (Orco, Ir8a, Ir76b, Ir25a). We find broad co-expression of these co-receptor genes in various combinations in olfactory neurons, challenging the current view of segregated olfactory families in the fly. In particular, Ir25a is expressed in the majority of olfactory neurons, including most Orco+ OSNs. In addition, the Ir8a and Ir25a knock-in lines help to distinguish two new OSN classes in the sacculus that target previously un-identified glomerular subdivisions in the posterior AL. Recordings in Ir25a mutant sensilla in Orco+ neurons reveal subtle changes in odor responses, suggesting that multiple chemoreceptor gene families could be involved in the signaling or development of a given OSN class. These data invite a re-examination of odor coding in Drosophila. We present a comprehensive model of co-receptor expression, which will inform future investigations of combinatorial chemosensory processing.

\section{Results}

\section{Generation and Validation of Co-Receptor Knock-in Lines}

We previously developed the HACK technique for CRISPR/Cas9-mediated in vivo gene conversion of binary expression system components, such as the conversion of transgenic Gal4 to QF2 (Brand and Perrimon, 1993; Jinek et al., 2012; Lin and Potter, 2016a, b; Potter et al., 2010; Riabinina et al., 2015; Xie et al., 2018). Here, we adapt this strategy for the efficient generation of targeted knock-ins (see Table 1 and Table 1-Source Data 1 for details). We chose 
to target the four chemosensory co-receptor genes to examine unmapped patterns of coreceptor expression in Drosophila melanogaster. We inserted a T2A-QF2 cassette and mCherry selection marker before the stop codon of the four genes of interest (Figure 2A and Figure 2-

152 Figure Supplement 1). By introducing the T2A ribosomal skipping peptide, the knock-in will

153 produce the full-length protein of the gene being targeted as well as a functional QF2

154 transcription factor (Figure 2A, Protein Products). This approach should capture the endogenous expression pattern of the gene under the control of the gene's native regulatory elements, while retaining the gene's normal function (Baena-Lopez et al., 2013; Bosch et al., 2020; Chen et al., 2020; Diao et al., 2015; Diao and White, 2012; Du et al., 2018; Gnerer et al., 2015; Gratz et al., 2014; Kanca et al., 2019; Lee et al., 2018; Li-Kroeger et al., 2018; Lin and Potter, 2016a; Vilain et al., 2014; Xue et al., 2014). We found that T2A-QF2 knock-ins were functional with some exceptions (See Figure 2-Figure Supplement 2 and Figure 2-Source Data 1). For example, Orco-T2A-QF2 knock-in physiology was normal, while a homozygous Ir25aT2A-QF2 knock-in exhibited a mutant phenotype. This suggests that the addition of the T2A peptide onto the C-terminus of Ir25a might interfere with its co-receptor function. QF2 driven GFP expression was detected in the adult antennae and maxillary palps (Figure

167 2B), as previously described (Larsson et al., 2004). We validated the Orco-T2A-QF2 knock-in 168 line with whole-mount antibody staining of maxillary palps (Figure 2C) and found a high degree 169 of correspondence between anti-Orco antibody staining and knock-in driven GFP in palpal 170 olfactory neurons (quantified in Table 2; see also Figure 2-Figure Supplement 3A - D for PCR 171 and sequencing validation of all knock-in lines). We confirmed the specificity of the anti-Orco 172 antibody by staining $\operatorname{Orco}^{2}$ mutant palps and found no labeling of olfactory neurons (Figure 2- 
Unlike Orco, Ir8a expression has previously been localized only to the antenna, to

175

176

177

178

olfactory neurons found in coeloconic sensilla and in the sacculus (Abuin et al., 2011). As expected, the knock-in line drove GFP expression only in the antenna (Figure 2D). To validate the Ir8a-T2A-QF2 knock-in line, we performed antibody staining on antennal cryosections and found the majority of cells to be double-labeled (Figure 2E, Table 2). There was no anti-Ir8a staining in control Ir8a ${ }^{1}$ mutant antennae (Figure 2-Figure Supplement 3F).

The Ir76b gene has previously been implicated in both olfaction and gustation and has been shown to be expressed in adult fly antennae, labella (mouthparts), legs, and wings (Abuin et al., 2011; Chen and Amrein, 2017; Croset et al., 2010; Ganguly et al., 2017; Hussain et al., 2016; Sanchez-Alcaniz et al., 2018; Zhang et al., 2013). We examined the Ir76b-T2A-QF2 knock-in line and found a similar pattern of expression in the periphery, with GFP expression in the antennae and labella (Figure 2F). Because an anti-Ir76b antibody has not previously been tested in fly antennae, we performed in situs on Ir76b-T2A-QF2 > GFP antennal cryosections to validate knock-in expression (Figure $2 \mathrm{G}$ ) and confirmed the specificity of the probe in $I r 76 b^{1}$ mutant antennae (Figure 2-Figure Supplement 3G).

Of the four Drosophila co-receptor genes, Ir25a has been implicated in the broadest array of cellular and sensory functions, from olfaction (Abuin et al., 2011; Benton et al., 2009; Silbering et al., 2011) and gustation (Chen and Amrein, 2017; Chen and Dahanukar, 2017; Jaeger et al., 2018), to thermo- and hygro-sensation (Budelli et al., 2019; Enjin et al., 2016; Knecht et al., 2017; Knecht et al., 2016), to circadian rhythm modulation (Chen et al., 2015). In the adult olfactory system, Ir25a expression has previously been reported in three types of structures in the antenna: coeloconic sensilla, the arista, and the sacculus (Abuin et al., 2011; Benton et al., 2009). We examined the Ir25a-T2A-QF2 knock-in line and found GFP expression in the adult antennae, labella, and maxillary palps (Figure $2 \mathrm{H}$ ). This was surprising because no Ionotropic Receptor expression has previously been reported in fly palps. To verify Ir25a protein expression in the maxillary palps, we performed whole-mount anti-Ir25a antibody staining in 
Ir25a-T2A-QF2 > GFP flies. We found broad Ir25a expression in palpal olfactory neurons

(Figure 2I), and a high degree of overlap between knock-in driven GFP expression and antibody staining (Table 2). As expected, there was no anti-Ir25a staining in $/ r 25 a^{2}$ mutant palps (Figure 2-Figure Supplement 3H).

We also examined co-receptor knock-in expression in Drosophila larvae. As in the adult stage, larval GFP expression was broadest in the Ir25a-T2A-QF2 and Ir76b-T2A-QF2 knock-in lines, with GFP labeling of neurons in the head and throughout the body wall (Figure 2-Figure Supplement 4). The Orco-T2A-QF2 knock-in line labeled only the olfactory dorsal organs in the larva, while the Ir8a-T2A-QF2 knock-in line did not have obvious expression in the larval stage (Figure 2-Figure Supplement 4). All subsequent analyses focused on the adult olfactory system.

\section{Expanded Expression of Olfactory Co-Receptors}

We next examined the innervation patterns of the four co-receptor knock-in lines in the adult central nervous system: the brain and ventral nerve cord (VNC) (Figure 3). Only two of the four lines (Ir25a and Ir76b) showed innervation in the VNC, consistent with the role of these genes in gustation in addition to olfaction (Figure 3-Figure Supplement 1A). In the brain, we compared the expression of each knock-in line (Figure 3A - D, green) to the corresponding transgenic Gal4 line (Figure 3A - D, orange) to examine differences in expression to what has previously been reported. Reporter alone controls for these experiments are shown in Figure 3-Figure Supplement 1B. All four knock-in lines innervated the ALs, and the Ir25a-T2A-QF2 and Ir76bT2A-QF2 lines additionally labeled the suboesophageal zone (SEZ), corresponding to gustatory axons from the labella (Figure 3C and D, arrowheads) (Hussain et al., 2016; Zhang et al., 2013).

The co-labeling experiments revealed that all four knock-ins label more glomeruli than previously reported (see Figure 3-Source Data 1 for AL analyses, Figure 3-Source Data 2 for traced examples of newly identified glomeruli in each knock-in line, and Table 3 for a summary of glomerular expression across all knock-in lines). Some glomeruli were not labeled 
225

226

227

228

229

230

231

232

233

234

235

236

237

238

239

240

241

242

243

244

245

246

247

248

249

250

consistently in all flies, which we define as variable expression (found in $<50 \%$ of brains examined).

Orco-T2A-QF2 labels seven 'non-canonical' glomeruli consistently, and one sporadically.

These include VM4 and VL2a, which correspond to Ir76b+ and Ir8a+ OSN populations, respectively (Figure 3A, outlines). We also found that the Orco knock-in sparsely but consistently labels the $\mathrm{V}$ glomerulus, which is innervated by $\mathrm{Gr} 21 \mathrm{a}+\mathrm{Gr} 63 \mathrm{a}+$ neurons (Figure 3A, box and zoom panel). Orco-T2A-QF2 also labels one Ir25a+ glomerulus consistently (VL1), three additional Ir8a+ glomeruli consistently (DL2d, DL2v, DP1I) and one variably (DC4). Surprisingly, when we crossed the transgenic Orco-Gal4 line (Larsson et al., 2004) to a stronger reporter (Shearin et al., 2014), we found that several of these additional glomeruli were weakly labeled by the transgenic line (Figure 3-Figure Supplement 2A). This suggests that there are OSN populations in which Orco is expressed either at low levels or in few cells, which might be why this expression was previously missed. We found this to be the case with the IrCo knockins, as well (described below).

There has been some inconsistency in the literature as to which glomeruli are innervated by Ir8a-expressing OSNs. For example, Silbering et al., 2011 note that their Ir8a-Gal4 line labels approximately ten glomeruli, six of which are identified (DL2, DP1I, VL2a, VL2p, DP1m, DC4). An Ir8a-Gal4 line generated by Ai et al., 2013 also labels about ten glomeruli, only two of which are identified (DC4 and DP1m) and which correspond to two glomeruli in Silbering et al., 2011. Finally, Min et al., 2013 identify three additional glomeruli innervated by an Ir8a-Gal4 line (VM1, VM4, and VC5) but not reported in the other two papers. DL2 was later subdivided into two glomeruli (Prieto-Godino et al., 2017), bringing the total number of identified Ir8a+ glomeruli to ten. However, we found that Ir8a-T2A-QF2 consistently labels twice as many glomeruli as previously reported. These additional glomeruli include an Ir25a+ glomerulus (VL1, Figure 3B), numerous Orco+ glomeruli (such as VA3 and VA5), and an Orco+/lr76b+ glomerulus (VC3) (see Figure 3-Source Data 1 for a full list of new glomeruli and Figure 3-Source Data 2 for 
251 outlined examples). Some of these additional glomeruli are weakly labeled by an Ir8a-Ga/4 line

252 (Figure 3-Figure Supplement 2B), but this innervation is only apparent when examined with a 253 strong reporter.

Of the four chemosensory co-receptor genes, the previously reported expression of Ir76b is the narrowest, with only four identified glomeruli (VM1, VM4, VC3, VC5) (Silbering et al., 2011). The Ir76b-T2A-QF2 knock-in labels more than three times this number, including several Orco+ glomeruli (such as DC3 and VC4), most Ir8a+ glomeruli (including DP1I), and one additional Ir25a+ glomerulus (VL1) (Figure 3C). As with Orco and Ir8a, some but not all of these glomeruli can be identified by crossing the transgenic Ir76b-Gal4 line to a strong reporter (Figure 3-Figure Supplement 2C). However, the Ir76b-Gal4 line labels additional glomeruli not seen in the knock-in (Figure 3-Figure Supplement 2C, Orco+ cluster). In total, the Ir76b-T2AQF2 knock-in labels 15 glomeruli consistently and two variably (Figure 3-Source Data 1 and 2). Ir25a-T2A-QF2 innervation of the AL was the most expanded compared to what has previously been reported. In addition to the novel expression we identified in the palps (Figure the antennae (Figure 3D). The extensive, dense innervation of the AL by Ir25a+ processes made identification of individual glomeruli difficult and necessitated further experiments to fully characterize this expression pattern (described in greater detail below). While it was previously reported that the transgenic Ir25a-Gal4 line labels only a subset of Ir25a+ neurons (compared to anti-Ir25a antibody staining), it was assumed that neurons not captured by the transgenic line

271 would reside in coeloconic sensilla, the arista or sacculus (the original locations for all IR+

272 OSNs) (Abuin et al., 2011). When we crossed Ir25a-Gal4 to a strong reporter, we found labeling 273 of a few Orco+ glomeruli (Figure 3-Figure Supplement 2D), but this was a small fraction of those 274 labeled by the knock-in. To further examine Ir25a expression and the potential co-expression of 275 multiple co-receptors in greater detail, we employed a combination of approaches including 276 single nucleus RNAseq (snRNAseq), immunohistochemistry, and optogenetics. 


\section{Confirmation of Co-Receptor Co-Expression}

278 The innervation of the same glomeruli by multiple co-receptor knock-in lines challenges the

279 previous view of segregated chemosensory receptor expression in Drosophila and suggests two

280 possible explanations: either the same olfactory neurons express multiple co-receptors (co-

281 expression), or different populations of olfactory neurons expressing different receptors

282 converge upon the same glomeruli (co-convergence). These scenarios are not necessarily

283 mutually exclusive. To examine these possibilities in a comprehensive, unbiased way, we

284 analyzed snRNAseq data from adult fly antennae (McLaughlin et al., 2021). Figure 4A shows

285 the expression levels of the four co-receptor genes in 20 transcriptomic clusters (tSNE plots

286 (Van der Maaten and Hinton, 2008), top row) which were mapped to 24 glomerular targets in

287 the brain (AL maps, bottom row). The proportion of cells in each cluster expressing the given

288 co-receptor gene is indicated by the opacity of the glomerular fill color, normalized to maximum

289 expression for that gene (see Methods and Figure 4-Source Data 1 for details on expression

290 normalization). The OSN classes to which these clusters map include Orco+ neurons (Figure

291 4A right column, teal), Ir25a+ neurons (Figure 4A right column, purple), Ir8a+ neurons (Figure

292 4A right column, pink), and GR+ neurons (Figure 4A right column, dark blue). They also include

293 example OSNs from all sensillar types (basiconic, intermediate, trichoid, coeloconic) as well as

294 from the arista and sacculus. The snRNAseq analyses confirmed expanded expression of all

295 four co-receptor genes into OSN classes not traditionally assigned to them. For example, Orco

296 and Ir25a are expressed in cluster 1, which maps to the $\mathrm{V}$ glomerulus (Gr21a+/Gr63a+).

297 Similarly, Ir8a and Ir76b are expressed in cluster 19 (VL1 glomerulus, Ir25a+), and Ir25a is

298 expressed in multiple Orco+ clusters (such as 15/VA2, 16/DL3, and 8/DC1).

299

The snRNAseq analyses confirm transcript co-expression in olfactory neurons in the

300 periphery. To demonstrate protein co-expression in OSNs, we performed anti-Orco antibody

301 staining on Ir25a-T2A-QF2 > GFP antennae and palps (Figure 4B). In the antennae, we found 
examples of Orco+ GFP+ double-labeled cells, as well as many cells that were either GFP+ or Orco+ (Figure 4B, top right panel). Interestingly, in the palps the vast majority of cells were double-labeled. We found a small population of palpal neurons which were only Orco+, and no neurons that were only GFP+ (Figure 4B, bottom right panel). These results are consistent with our anti-Ir25a staining experiments in the palps (Figure 2I), which showed that most of the $\sim 120$ palpal OSNs express Ir25a protein.

The snRNAseq data from the antennae and peripheral immunohistochemical experiments in the palps helped to identify some of the novel OSN populations expressing Ir25a. We extended these analyses with co-labeling experiments in which we combined transgenic OrX-, IrX-, or GrX-Gal4 lines labeling individual glomeruli with the Ir25a knock-in to verify the glomerular identity of Ir25a+ axonal targets in the AL. Two examples are shown in Figure 4C (one antennal and one palpal OSN population), and the full list of OSN classes checked can be found in Figure 4-Source Data 2.

For some OSN classes not included in the snRNAseq dataset for which co-labeling experiments yielded ambiguous results, we employed an optogenetic approach. We used the Ir25a-T2A-QF2 knock-in to drive expression of QUAS-CsChrimson, a red-shifted channelrhodopsin (Klapoetke et al., 2014), and performed single sensillum recordings (SSR) from sensilla previously known to house only Orco+ neurons. If these neurons do express Ir25a, then stimulation with red light should induce neuronal firing. We recorded from ab3 sensilla, which have two olfactory neurons ( $A$ and $B$; indicated with blue and green dots, respectively, in Figure 4D). Ab3A neurons innervate DM2 and ab3B neurons innervate VM5d. Both neurons responded to pulses of $627 \mathrm{~nm}$ light at various intensities in a dose-dependent manner, confirming Ir25a expression in these neurons. No light-induced responses were found in control flies not fed all-trans retinal (-ATR), a necessary co-factor for channelrhodopsin function (see Methods). We used similar optogenetic experiments to examine Ir25a expression in OSN classes innervating DM4 (ab2A, Or59b+) and DM5 (ab2B, Or85a/Or33b+) (Figure 4-Figure 
Supplement 1A-B), as well as D (ab9A, Or69aA/aB+) and VA3 (ab9B, Or67b+) (Figure 4-Figure

Supplement 1C-D). These experiments indicated that Ir25a is expressed in ab2A (DM4) and ab9B (VA3) neurons, but not ab2B (DM5) or ab9A (D) neurons (see also Figure 4-Source Data 2 and 3). Results of these experiments are summarized in Table 3.

\section{Identification of New OSN Classes}

333 The co-receptor knock-ins allowed us to analyze the olfactory neuron innervation patterns for all antennal lobe glomeruli. Interestingly, the Ir8a-T2A-QF2 and Ir25a-T2A-QF2 knock-ins strongly labeled a previously uncharacterized posterior region of the AL. By performing a co-labeling experiment with Ir41a-Gal4, which labels the VC5 glomerulus, we narrowed down the anatomical location of this region and ruled out VC5 as the target of these axons (Figure 5A). While both knock-ins clearly labeled VC5, they also labeled a region lateral and slightly posterior to it (Figure 5A, outline). We performed additional co-labeling experiments with Ir8a-T2A-QF2 and various Gal4 lines labeling all known posterior glomeruli to confirm that this $\mathrm{AL}$ region did not match the innervation regions for other previously described OSN populations (Figure 5portion of the recently identified Rh50+ ammonia-sensing olfactory neurons (Vulpe et al., 2021a). Co-labeling experiments with Rh50-Gal4 and Ir8a-T2A-QF2 confirmed that they indeed partially overlapped (Figure 5B). We determined that these Rh50+ olfactory neurons mapped to a portion of the VM6 glomerulus, with the strongly Ir8a+ region innervating the 'horn' of this

347 glomerulus. The difference in innervation patterns between Ir8a+ and Rh50+ neurons in this $\mathrm{AL}$

348 region suggested at least two different subdivisions or OSN populations within this VM6

349 glomerulus. In fact, in between the main body of VM6 and the Ir8a+ horn there appeared to be a 350 third region (Figure 5B, horn outlined in white, other two regions outlined in blue). We

351 designated these subdivisions VM6I, VM6m, and VM6v (for lateral, medial, and ventral). We 352 coordinated the naming of this glomerulus with recent connectomics analyses of the entire fly 
antennal lobe (Schlegel et al., 2021). In this connectomics study, dendrites of olfactory projection neurons were found to innervate the entire region described here as VM6I, VM6m, and VM6v. No projection neurons were identified to innervate only a subdomain. As such, the new VM6 nomenclature reflects this unique subdivision of a glomerulus by olfactory sensory neurons but not second order projection neurons.

We sought to determine the identity of the olfactory neurons that might be innervating these three VM6 subdivisions. Rh50+ neurons can be found in two regions of the antenna: ac1 coeloconic sensilla and the sacculus (Figure 5C) (Vulpe et al., 2021a). The shape of the VM6v subdomain most closely matches the glomerulus described as VM6 by previous groups (e.g. Couto et al., 2005; Endo et al., 2007), which had been suggested to be innervated by coeloconic sensilla (Chai et al., 2019; Li et al., 2016). In addition, antibody staining had previously shown that Rh50+ ac1 neurons broadly co-express Ir25a but generally not Ir8a (Vulpe et al., 2021a). This suggested that the other VM6 subdomains might be innervated by the Rh50+ sacculus olfactory neurons. Antibody staining in Rh50-Gal4>GFP antennae confirmed co-expression with both Ir25a protein (broad overlap) and Ir8a protein (narrow overlap) in the third chamber of the sacculus (Figure 5D; quantified in Table 4). Most sacculus neurons appear to be Ir25a+, and in contrast to the Ir8a knock-in, the three VM6 subdivisions are all strongly innervated by the Ir25a knock-in (Figure 5A). Two previously described OSN populations in the third chamber of the sacculus had been characterized to express Ir8a along with Ir64a and innervate the DP1m and DC4 glomeruli (Ai et al., 2013; Ai et al., 2010). To

373 demonstrate that the Rh50+ Ir8a+ sacculus neurons represented a distinct olfactory neuron 374 population, we performed immunohistochemistry experiments in Rh50-Gal4>GFP antennae with an anti-Ir64a antibody (Figure 5E, top), and in Ir64a-Gal4>GFP antennae with an anti-Ir8a antibody (Figure 5E, bottom). These experiments confirmed a new, distinct population of Ir8a+ 377 Ir64a- cells in the sacculus. 
The VM6I olfactory projections are difficult to identify in the hemibrain connectome (Scheffer et al., 2020) due to the medial truncation of the AL in that dataset (see Schlegel et al., 2021 for additional details). Here, we used FlyWire (Dorkenwald et al., 2020), a recent segmentation of a full adult fly brain (FAFB) (Zheng et al., 2018), to reconstruct the VM6 OSN projections in both left and right AL. Synapse-based hierarchical clustering (syNBLAST) (Buhmann et al., 2021) of the VM6 OSNs demonstrated the anatomical segregation into three distinct subpopulations: VM6I, VM6m and VM6v (Figure 5F). This subdivision was subsequently confirmed in a re-analysis of the VM6 glomerulus in the hemibrain dataset (Schlegel et al., 2021). Olfactory neurons innervating VM6I were strongly Ir8a+, while olfactory neurons innervating VM6m and VM6v were weakly and sparsely Ir8a+ (see Figure 3-Source Data 2, page 3). This pattern may be due to Ir8a expression in only one or a few cells. Interestingly, this sparse Ir8a+ expression in olfactory neurons targeting VM6m and VM6v may be sexually dimorphic: we found that male brains had stronger and more frequent Ir8a+ innervation in these two glomeruli compared to female brains (see also Figure 3-Source Data 1). experiments, we propose a model of the anatomical locations and molecular identities of the olfactory neurons innervating the VM6 subdivisions (Figure 5F). All VM6 subdivisions broadly express Rh50 and Ir25a; the VM6v OSNs are housed in ac1 sensilla and express Ir8a either weakly or only in a small subset of neurons; both the VM6m and VM6I OSNs are found in the sacculus and can be distinguished by their levels or extent of Ir8a expression, with VM6I neurons being strongly Ir8a+. Because all three VM6 subdivisions share the same downstream projection neurons, this AL region has been classified as a single glomerulus (Schlegel et al., 400 2021). We maintain this convention here, for a total of $58 \mathrm{AL}$ glomeruli. It is possible that this 401 number may need to be re-evaluated in the future, and the three VM6 subdivisions reconsidered as bona fide separate glomeruli (bringing the OSN glomerular total to 60). Such a separation 
might be warranted if it is found that these OSN populations express different tuning receptors, and those receptors respond to different odorants.

Table 3 summarizes the chemosensory receptor expression patterns for all four coreceptor knock-in lines across all OSNs, sensillar types, and glomeruli. For clarity, this summary considers the newly identified OSN populations described here separately. We find that OrcoT2A-QF2 consistently labels 45 total glomeruli out of 58 (7 more than previously reported); Ir8aT2A-QF2 consistently labels 18 glomeruli (8 more than previously identified); Ir76b-T2A-QF2 consistently labels 15 glomeruli (11 more than previously identified); and Ir25a-T2A-QF2 consistently labels 51 glomeruli (39 more than previously identified).

\section{Co-receptor Contributions to Olfactory Neuron Physiology}

How might the broad, combinatorial co-expression of various chemosensory families affect olfactory neuron function? To begin to address this question we examined olfactory responses in neuronal populations co-expressing just two of the four chemosensory receptor families (Orco and Ir25a). We chose to test eight OSN classes previously assigned to the Orco+ domain that we found to have strong or intermediate Ir25a expression - two in the antennae, and six in the maxillary palps. The two antennal OSN classes are found in the same ab3 sensillum (ab3A, Or22a/b+, DM2 glomerulus; and ab3B, Or85b+, VM5d glomerulus). The six palpal OSN classes represent the entire olfactory neuron population of the maxillary palps (pb1A, Or42a+, VM7d; pb1B, Or71a+, VC2; pb2A, Or33c/Or85e+, VC1; pb2B, Or46a+, VA7I; pb3A, Or59c+, VM7v; pb3B, Or85d+, VA4). In both the antennae and the palps, we compared the olfactory responses of OSNs to a panel of 13 odorants in three genotypes: wildtype, Ir $25 a^{2}$ mutant, and Orco ${ }^{2}$ mutant flies. This panel included odorants typically detected by ORs, such as esters and aromatics, and odorants typically detected by IRs, such as acids and amines (Silbering et al., 2011). In the previously accepted view of olfaction in Drosophila, Orco+ neurons express only Orco/OrX receptors, and all olfactory responses in the neurons can be attributed to these 
428

429

430

431

432

433

434

435

436

437

438

439

440

441

442

443

444

445

446

447

448

449

450

451

452

453

receptors. Thus, in an $I r 25 a^{2}$ mutant background, there should be no difference in olfactory responses from wildtype if either a) Ir25a is not expressed in these neurons, or b) Ir25a is expressed, but is not playing a functional role in these neurons. In an Orco $^{2}$ mutant background, there would be no trafficking of Orco/OrX receptors to the dendritic membrane, and no formation of functional ion channels (Benton et al., 2006; Larsson et al., 2004). Thus, in the traditional view of insect olfaction, $\mathrm{Orco}^{2}$ mutant neurons should have no odor-evoked activity. However, in the new co-receptor co-expression model of olfaction, if Ir25a is contributing to olfactory responses in Orco+ neurons, then mutating this co-receptor might affect the response profiles of these neurons. Similarly, $\operatorname{Orco}^{2}$ mutant neurons that co-express Ir25a might retain some odorevoked activity.

We first examined olfactory responses in palp basiconic sensilla. In the palps, three types of basiconic sensilla (pb1, pb2, and pb3) contain two neurons each (A and B) (Figure 6A), for a total of six OSN classes (Couto et al., 2005; de Bruyne et al., 1999; Fishilevich and Vosshall, 2005; Goldman et al., 2005; Ray et al., 2008; Ray et al., 2007). We found robust responses to several odorants in our panel in both the wildtype and $/ r 25 a^{2}$ mutant flies, including odorants like 1-octen-3-ol typically considered as an OR ligand (Figure 6B), and IR ligands like pyrrolidine. Neither odor-evoked nor spontaneous activity was detected in the $\operatorname{Orco}^{2}$ mutant (Figure 6B, bottom row; see also Figure 6-Figure Supplement 1A). This was true of all sensilla tested in the palps. The SSR experiments in Figure 6A-D were performed at $4-8$ DPE. We recently discovered that neurodegeneration of $\mathrm{Orco}^{2}$ mutant olfactory neurons occurs in the palps by 6 DPE (Task and Potter, 2021), which could potentially confound our interpretation. We repeated the experiments in young (1 - 3 DPE) flies but similarly detected neither odorevoked activity nor spontaneous activity in mutant palpal neurons (Figure 6-Figure Supplement 1B). There was also no spontaneous or odor-evoked activity in an $I r 25 a^{2}$; Orco double mutant (Figure 6-Figure Supplement 1C). This suggests one of three possibilities: first, Orco $^{2}$ mutant neurons in the palps could already be dysfunctional at this early stage, despite not yet showing 
cell loss, and Ir25a-dependent activity is not sufficient to maintain either baseline or stimulusinduced activity; second, Ir25a function may be Orco-dependent in these cells, or act downstream of Orco, such that loss of Orco function affects Ir25a function; third, we did not stimulate neurons with an Ir25a-dependent odorant. The latter possibility would not, however, explain why there is no spontaneous activity in these cells. Future experiments will be needed to address these possibilities. Given the lack of neuronal activity in the Orco ${ }^{2}$ mutant, we focused subsequent analyses in the palps on the two other genotypes: wildtype and Ir25a'. mutant compared to the wildtype (Mann-Whitney $U$ test, $p=0.0016$ ), as was the response to methyl salicylate $(p=0.0177)$, while the response to ethyl acetate was higher in wildtype $(p=$ 0.008) (Figure 6C; see Figure 6-Source Data 1 for results of all statistical analyses). The differences in responses across all six OSN classes in the palps between wildtype and $/ r 25 a^{2}$ mutant flies are summarized in Figure 6D. In each neuron class, we found one to three odorants whose response profiles differed between the two genotypes. However, the specific stimuli lower responses in the mutant (higher in the wildtype) in pb3B. Interestingly, when we examined a list of candidate IrX tuning receptors (Li et al., 2021) in the palps using in situs, we did not find expression (see Figure 6-Figure Supplement 2 and Figure 6-Source Data 2). This suggests that Ir25a may not be functioning as a traditional co-receptor in Orco+ olfactory neurons in the palps

474 (an expanded role for Ir25a beyond co-reception has previously been suggested; see Budelli et al., 2019; Chen et al., 2015). 
Supplement 1D for additional example traces). Although there are two Orco+ neurons in this

481

482

483

484

485

486

487

488

489

490

491

492

493

494

495

496

497

498

499

500

501

502

503

504

sensillum, we consistently observed only a single spike amplitude in the Orco ${ }^{2}$ mutant. Thus, we cannot determine at this time whether this activity arises from the A or B neuron. We occasionally observed small responses ( $\leq 10 \Delta$ spikes/sec) in the Orco ${ }^{2}$ mutant; however, across all flies tested, these responses were not significantly different from the mineral oil control (Figure 6I; statistical analyses can be found in Figure 6-Source Data 1). For these reasons, $\mathrm{Orco}^{2}$ mutant flies were excluded from the analyses in Figure 6G and Figure 6H. As in the palps, we found significant differences in the responses of both ab3A and ab3B neurons to some odorants between the two genotypes. Comparison of all ab3A responses between wildtype and $I r 25 a^{2}$ mutant are shown in Figure $6 \mathrm{G}$, and results from both the $A$ and $B$ neurons are summarized in Figure 6H (Mann-Whitney $U$, as in Figure 6A-C; see Figure 6Source Data 1 for all analyses). In the ab3A neuron, the wildtype showed higher responses to propionic acid $(p=0.0441), 1$-octen-3-ol $(p=0.0004)$, phenylacetaldehyde $(p=0.015)$, and pentyl acetate $(p=0.0164)$. Interestingly, two of these four odorants are typically associated with IRs (propionic acid and phenylacetaldehyde). In the ab3B neuron, only two odorants elicited significantly different responses between the wildtype and $I r 25 \mathrm{a}^{2}$ mutant: propionic acid (response higher in wildtype, as with ab3A; $p=0.0388$ ), and pentyl acetate (response higher in mutant, in contrast to ab3A; $p=0.0385)$. While responses to propionic acid are small in both ab3 neurons, they are abolished in the $I r 25 a^{2}$ mutant background (Kruskal-Wallis with uncorrected Dunn's comparing odorant responses to mineral oil control; ab3A $p=0.3957$; ab3B $p=0.5184$ ), suggesting that propionic acid detection in ab3 may be Ir25a-dependent.

\section{The Co-Receptor Co-Expression Map of Olfaction in Drosophila}

Co-receptor co-expression of insect chemosensory receptors suggests multiple receptors may influence the response properties of an olfactory neuron, as we have shown in ab3 and palpal sensilla. To aid future investigations of co-receptor co-expression signaling, we synthesized our 
505

506

507

508

509

510

511

512

513

514

515

516

517

518

519

520

521

522

523

524

525

526

527

528

529

530

results (Table 3 ) into a comprehensive new map of the AL. Figure 7 summarizes the expression patterns of all the co-receptor knock-in lines and presents a new model for chemosensory receptor expression in Drosophila melanogaster. In Figure 7A, the expression pattern of each knock-in line is presented separately (see also Figure 3-Source Data 1). The new AL map is updated with the recent re-classification of VP1 into three glomeruli (Marin et al., 2020) and indicates the new VM6 subdivisions. In Figure 7A, the original glomerular innervation pattern for each co-receptor is shown in green, with new innervation revealed by the T2A-QF2 knock-in lines color coded by intensity: strongly labeled glomeruli are in orange, intermediate glomeruli in yellow, and weakly labeled glomeruli are in pink. Glomeruli labeled in $<50 \%$ of brains examined are designated variable (grey), and glomeruli not labeled by the given knock-in are in white. The new VM6v, VM6m, and VM6I subdivisions are labeled with grey stripes.

In the previous model of olfaction in Drosophila, the Orco/OR domain primarily occupied the anterior $\mathrm{AL}$, while the IR domains innervated more posterior glomeruli. While the former is still, for the most part, accurate (Figure 7A, Orco), the latter is not: both Ir8a-T2A-QF2 and Ir76b-T2A-QF2 label several more anterior glomeruli (such as VA3 or VA6), and Ir25a-T2A-QF2 labels the majority of glomeruli throughout the anterior to posterior axis (Figure 7A, Ir25a). The expansion of the Ir25a+ domain is the most dramatic of the four co-receptors: previously, Ir25a+ glomeruli accounted for 21\% of the AL (12/58 glomeruli) (Enjin et al., 2016; Frank et al., 2017; Marin et al., 2020; Silbering et al., 2011); the Ir25a-T2A-QF2 knock-in consistently labels 88\% of the $\mathrm{AL}$ (51/58 glomeruli, excluding variable). This represents a greater than fourfold expansion. Similarly, the number of Ir76b+ glomeruli increased more than threefold, from $7 \%$ of the $A L$ (4/58 glomeruli) (Silbering et al., 2011), to $26 \%$ (15/58, excluding variable). The Ir8a+ domain has nearly doubled, from $17 \%$ of the AL originally (10/58 glomeruli) (Silbering et al., 2011) to $31 \%(18 / 58$ glomeruli, excluding variable). The most modest increase in reported expression is in the Orco+ domain: from $66 \%$ of the $\mathrm{AL}$ (38/58 glomeruli) (Couto et al., 2005; Fishilevich and Vosshall, 2005), to $78 \%$ (45/58, excluding variable). 
The expression overlap in the AL of the four co-receptor families is summarized in the

532 Venn diagram shown in Figure 7B (excluding the variably labeled glomeruli from Figure 7A).

533 The table at the right lists the names of the glomeruli which correspond to the sections of the

534 Venn diagram. This analysis reveals 9 glomeruli labeled by all four knock-in lines; furthermore, it

535 shows that the Ir8a+ and Ir76b+ domains do not have glomeruli unique to them. Most of the AL

536 is innervated by Orco+ Ir $25 a+$ neurons ( 25 glomeruli that are only Orco+ Ir $25 a+$, plus an

537 additional 13 that have Orco, Ir25a, and one or both other co-receptors). The Orco+ and Ir25a+

538 domains reveal glomeruli unique to them (6 glomeruli that are only Orco+, 7 glomeruli that are

539 only Ir25a+). Expression analyses also reveal that Ir8a does not co-express with Orco alone or

$540 \quad$ Ir76b alone.

A unified AL map organized by chemosensory gene families (ORs, IRs, and GRs) is

542 shown in Figure 7C (right panel), and the left two panels extend this information into the

543 periphery. Here we include the GR+ innervation of the $\mathrm{V}$ glomerulus. However, a knock-in line

544 for either Gr21a or Gr63a does not currently exist; thus, it is possible these receptors (as well as

545 other poorly characterized antennal GRs) might also be more broadly expressed than previous

546 transgenic lines indicate (Fujii et al., 2015; Menuz et al., 2014). All four OR and IR co-receptors

547 are expressed in the antenna, while olfactory neurons in the palps express Orco and Ir25a

548 (Figure 7C, left panel). In the antennae, there are many different classes of OSNs expressing

549 various combinations of chemosensory receptors and co-receptors: there are Orco+ only

550 neurons (Figure 7C, middle panel, \#2), such as those innervating the VM2 and VM3 glomeruli

551 (teal); IrCo+ only neurons (purple), which include neurons expressing one, two, or all three IR

552 co-receptors (such as VP2, VM6v, or DP1m respectively) (Figure 7C, middle panel, \#1); and

553 neurons expressing both Orco and $\mathrm{IrCo}(\mathrm{s})$ (teal and purple stripe) (Figure 7C, middle panel, \#3

554 and 4).

$555 \quad$ The expression data suggest that different sub-populations of olfactory neurons might be

556 targeting a shared glomerulus. Our data indicate that both Orco+ and Ir25a+ neurons innervate 
557 the GR+ V glomerulus (dark blue; see also Figure 7A). Based on the sparse innervation of the $\mathrm{V}$ 558 glomerulus by the Orco-T2A-QF2 knock-in (Figure 3A) and the lower expression levels in the 559 snRNAseq data (Figure 4A), we hypothesize that Orco may be expressed in only a subset of $560 \mathrm{Gr} 21 \mathrm{a} / \mathrm{Gr} 63 \mathrm{a}+$ neurons. This contrasts with the Ir25a-T2A-QF2 knock-in, which appears to label 561 most Gr21a/Gr63a+ neurons. Thus, two sub-populations of neurons may be co-converging 562 upon the same V glomerulus: neurons that express Gr21a/Gr63a and Ir25a (dark blue and 563 purple stripes), and neurons that express Gr21a/Gr63a, Ir25a and Orco (dark blue, purple, and 564 teal stripes) (Figure 7C, middle panel, \#5). Such co-convergence has recently been shown in 565 the olfactory system of Aedes aegypti mosquitoes (Younger et al., 2020). Similarly, the sparse 566 Orco-T2A-QF2 knock-in innervation of the DP1I glomerulus suggests that there are OSN 567 populations expressing mostly IRs, but with a subset of neurons that additionally express Orco 568 (Figure 7C, middle panel, \#3). The converse may also be possible (Figure 7C, middle panel, \#4): OSN populations which have some neurons expressing only Orco, and a subset expressing both Orco and $I r C o(s)$ co-converging onto the same glomerulus. There is some evidence for this

571 in the palps, based on our anti-Orco and anti-Ir25a antibody staining (Figure 2C and I; Figure

572 4B; Table 2). The snRNAseq data suggest this may also be the case in the antennae (see

573 Figure 4-Source Data 1).

\section{Discussion}

575 Here we present evidence in Drosophila melanogaster of widespread chemosensory coreceptor co-expression in the olfactory system, contrasting a previously accepted view of

577 segregated, mutually exclusive olfactory domains. By generating targeted knock-ins of the four 578 main chemosensory co-receptors (Orco, Ir8a, Ir76b, Ir25a), we demonstrate that all four co579 receptors have broader olfactory neuron expression than previously appreciated. Co-expression 580 of these co-receptors was found in the periphery and in the central projections to the brain. The 581 Ir25a co-receptor was previously thought to be expressed only in a small subset of olfactory 
neurons (coeloconic, sacculus, and arista neurons), but we present evidence that it is expressed in subsets of nearly all OSN classes that innervate the majority of the fly ALs. We further demonstrate that co-expression of chemosensory co-receptors may have functional relevance for olfactory neurons, and that the Ir25a co-receptor may be involved in modulating the activity of some Orco+ OSNs, both in the antennae and maxillary palps. We present a new AL map which will aid future inquiries into the role that specific chemoreceptor co-expression plays in distinct OSN populations.

A model for OR/IR segregation was initially supported by developmental evidence. Two pro-neural genes specify the development of sensory structures on the antennae: amos and atonal. Amos mutants lack basiconic and trichoid sensilla, while atonal mutants do not develop coeloconic sensilla, the arista, or the sacculus (Goulding et al., 2000; Gupta and Rodrigues, 1997; Jhaveri et al., 2000a; Jhaveri et al., 2000b). It was observed that amos mutants lose Orco expression while retaining Ir25a expression (Benton et al., 2009). Our results generally do not conflict with this view. In the traditional segregated model of the fly olfactory system, it was presumed that atonal mutant antennae would show the reverse pattern: loss or IrCo expression but not Orco expression. However, our co-receptor knock-in expression results suggest that atonal mutants should have significant IrCo expression, particularly of Ir25a. This was indeed found to be the case in RNAseq analyses performed on atonal mutant antennae, which showed that both Ir25a and Ir76b expression, but not Ir8a expression, remained (Menuz et al., 2014). Based upon the strength of the corresponding glomerular innervations, it does appear that the previously reported Ir25a+ neurons have stronger or more consistent Ir25a expression, while the new Ir25a+ olfactory neurons in the antennae reported here (e.g. OR-expressing OSNs) are often weakly or stochastically labeled. This might also explain why Ir25a expression was initially overlooked in these Orco+ neural populations. The developmental pattern is different in the maxillary palps, where it is atonal and not amos which is required for development of the basiconic sensilla (Gupta and Rodrigues, 1997). Interestingly, the Ir25a knock-in expression 
corresponds well with the atonal developmental program across the olfactory appendages: the strongest expression of Ir25a is in coeloconic sensilla, but outside of these sensilla the strongest and most consistent Ir25a expression is in palp basiconic sensilla.

Chemosensory co-receptor co-expression may help clarify previously confounding observations regarding Drosophila odor coding. For example, olfactory neurons in the sacculus that express the Ir64a tuning receptor along with the Ir8a co-receptor project to two different glomeruli: DC4 and DP1m (Ai et al., 2013; Ai et al., 2010). These two glomeruli exhibit different olfactory response profiles, with DC4 being narrowly tuned to acids or protons, while DP1m is more broadly tuned. The molecular mechanism for these differences was previously unclear as it was thought these neurons expressed the same receptors. However, the co-receptor knock-in data presented here reveal that the Ir64a+ OSN sub-populations do in fact express different combinations of chemoreceptors: in addition to the Ir8a co-receptor, neurons innervating DC4 express Ir25a (and occasionally Orco) (see Figure 7). In contrast, neurons innervating DP1m express Ir8a, Ir25a, and Ir76b. Thus, perhaps it is Ir76b expression in DP1m-targeting Ir64a+ neurons which makes them olfactory generalists. This idea is supported by experiments in which Ir64a was misexpressed in neurons targeting the VM4 glomerulus, conferring a DP1mlike, rather than a DC4-like, response profile to VM4 (Ai et al., 2010). We show here that VM4targeting neurons express Ir8a, in addition to Ir25a and Ir76b (as well as Orco): thus, molecularly, the VM4 neuron profile is more similar to DP1m (co-expressing all IrCos) than DC4 (co-expressing two IrCos), and the key distinguishing component appears to be Ir76b. It would be interesting to repeat such misexpression experiments in an $I r 76 b^{-}$mutant background to test this hypothesis.

The widespread expression of Ir25a in the fly olfactory system raises the possibility that it might have roles in addition to its function as an IrX co-receptor. For example, Ir25a has been found to play a developmental role in forming the unique structure of Cold Cells in the arista (Budelli et al., 2019). Evolutionary studies also suggest that Ir25a is the most ancient of all the 
634 insect chemosensory receptors (Croset et al., 2010), and the currently broad expression might 635 reflect its previous ubiquitous role in chemosensory signaling. Ir25a may also be involved in 636 downstream olfactory signal transduction or amplification, as suggested by the work presented 637 here, and as has recently been shown for a pickpocket ion channel ( $\mathrm{Ng}$ et al., 2019).

638 Experiments addressing potentially expanded roles for Ir25a in olfactory neurons will be aided 639 by the new chemosensory co-receptor map presented here. While we demonstrate here that multiple chemosensory co-receptors can be co641 expressed in the same olfactory neurons, it remains to be determined if this also applies to 642 tuning (odor-binding) receptors. Previous studies suggest that OrX tuning receptors are generally limited to a single class of olfactory neurons, with the sole exception being Or33b

644 (Couto et al., 2005; Fishilevich and Vosshall, 2005). However, many IrX tuning receptors remain to be characterized and could be co-expressed in multiple olfactory neurons. For example, recordings from ab1, ab3, and ab6 sensilla indicate responses to the typical IR odors 1,4-

647 diaminobutane, ammonia, and butyric acid, respectively (de Bruyne et al., 2001), suggesting tuning IrXs may be involved. We show that Ir25a plays a functional role in Orco+ neurons in the antennae and palps; this suggests that these Orco+ neurons could also express as yet unidentified ligand binding IrXs. The recent release of the whole fly single-cell atlas, which

651 includes RNAseq data from maxillary palps, allowed us to identify 6 IRs that might be expressed 652 in palpal OSNs (Ir40a, Ir51a, Ir60a, Ir62a, Ir76a, Ir93a) (Li et al., 2021). However, in situ 653 analyses for these 6 IRs in the maxillary palps did not detect a signal (see Figure 6-Figure 654 Supplement 2 and Figure 6-Source Data 2). This suggests that Ir25a in the palps may be 655 playing a role independent of its role as a co-receptor, as discussed above, or that a tuning IrX 656 was missed by the RNAseq analyses. In antennal ab3 sensilla, we did find one odorant 657 (propionic acid) which elicited a small response in wildtype neurons, and no response in $/ r 25 a^{2}$ 658 mutant neurons. It is possible that other antennal Orco+ OSNs might utilize IR chemoreceptors 659 for signaling. For example, the ac3B neuron, which expresses Or35a/Orco and all IR co- 
660

661

662

663

664

665

666

667

668

669

670

671

672

673

674

675

676

677

678

679

680

681

682

683

684

685

receptors, has recently been suggested to utilize an unidentified IrX to mediate responses to phenethylamine (Vulpe et al., 2021b). The chemoreceptor expression patterns revealed in this work will help the search for olfactory neurons that may utilize multiple chemosensory families for odor detection.

Based on the co-receptor innervation patterns in the antennal lobes, we identified a glomerulus, VM6, that is uniquely partitioned by different olfactory sensory neurons (Figure 5) (also see Schlegel et al., 2021). The co-receptor expression patterns allowed us to pinpoint the likely origin of the innervating OSNs. Since the VM6 glomerulus was labeled by both the Ir25aT2A-QF2 and Ir8a-T2A-QF2 knock-in lines, the cell bodies of these neurons had to reside in the antenna; furthermore, since we did not find Ir8a-T2A-QF2 labeling of the arista, these neurons were likely to be either in coeloconic sensilla or in the sacculus. Indeed, we determined the VM6 glomerulus to be innervated by the newly discovered Rh50+ Amt+ olfactory neurons which originate in the sacculus and ac1 sensilla (Vulpe et al., 2021a). Based on our results, Rh50+ Amt+ sacculus neurons are further subdivided into those that strongly express Ir8a, which innervate the VM6I region, and those that weakly or infrequently express Ir8a, which innervate VM6m. The functional consequences of this unusual subdivision by olfactory neurons for a glomerulus, and how this relates to the fly's olfactory perception of ammonia or other odorants, remains to be determined. These results also highlight the value of exploring chemosensory receptor expression patterns even in the era of connectomics, as the VM6 glomerulus and its subdivisions were not easily identifiable in prior connectomics reconstructions of the entire antennal lobe (Bates et al., 2020; Scheffer et al., 2020; Schlegel et al., 2021).

Co-expression of chemosensory co-receptors might function to increase the signaling capabilities of an olfactory neuron. For example, the signaling of an Orco+ olfactory neuron may be guided primarily by the tuning OrX, and the sensitivity range extended to include odors detectable by an IrX. Co-expression might also allow synergism, such that weak activation of a co-expressed receptor could increase neuronal activity to levels sufficient to drive behavior. This 
might be useful in tuning behavioral response to complex odors, such that certain combinations

687 of odors lead to stronger olfactory neuron responses. Alternatively, a co-expressed receptor

688 inhibited by odorants might be able to attenuate a neuron's response to odor mixtures. Co-

689 expressed chemosensory receptors might also modulate the perceived valence of an olfactory

690 stimulus through the same olfactory circuit or channel. For example, a recent study showed that

$691 \mathrm{CO}_{2}$, which is normally aversive to flies, can be attractive in certain contexts (van Breugel et al.,

692 2018). While the aversive response requires the known $\mathrm{CO}_{2}$ receptors, Gr21a and Gr63a, the

693 attractive response was shown to be Ir25a-dependent. However, previous calcium imaging

694 experiments in the $\mathrm{AL}$ have shown that $\mathrm{CO}_{2}$ primarily activates a single glomerulus, the $\mathrm{V}$

695 glomerulus, which receives inputs from the Gr21a+/Gr63a+ neurons (Suh et al., 2004). Our Ir25a-T2A-QF2 knock-in line reveals that Ir25a is expressed in this OSN class. This raises the

697 possibility that the same neurons, through a different chemoreceptor, could drive both attractive

698

699

700

701

702

703

704

705

706

707

708

709

and aversive behavior in different contexts (MacWilliam et al., 2018). Co-expression of

chemosensory receptors could be a mechanism to increase the functional flexibility of a numerically limited olfactory system.

Drosophila often serve as a model for many other insect olfactory systems, and information gleaned from Drosophila is frequently extrapolated to other insects (for example, DeGennaro et al., 2013; Fandino et al., 2019; Riabinina et al., 2016; Trible et al., 2017; Yan et al., 2017). The work presented here raises the possibility that other insects may also exhibit coexpression of chemosensory co-receptors. New work in Aedes aegypti mosquitoes suggests that this may indeed be the case: mosquito olfactory neurons can co-express $\mathrm{Orco} / \mathrm{IrCo} / \mathrm{Gr}$ receptors; in the $\mathrm{CO}_{2}$-sensing olfactory neuron, this co-expression functionally expands the range of activating odors (Younger et al., 2020). This suggests that co-expression of chemosensory co-receptors may be an important feature of insect olfactory neurons. 


\section{Acknowledgements}

711 We thank E. Marr and K. Robinson for splinkerette genetic mapping, Y.-T. Chang for cloning of

712 pHACK ${ }^{1 r 8 a}$ components, O. Riabinina for initial QUAS-CsChrimson characterization, S. Maguire

713 for preliminary SSR experiments, P. Mohapatra for RNAseq insights, D. Baktash for help with

714 figures, J. Konopka for advice on statistical analyses, S. Shankar for discussion of AL mapping,

715 and R. Mann for providing lab resources. We would like to thank E.C. Marin and M. Costa for

716 discussions regarding posterior AL glomeruli. We are also grateful to the Seung and Murthy labs

717 for access to the flywire.ai reconstruction community. Many thanks to the following labs for

718 sharing antibody and fly stock reagents: Leslie B. Vosshall (Rockefeller), Richard Benton

719 (UNIL), Paul Garrity (Brandeis), Marco Gallio (Northwestern), Greg Suh (NYU/KAIST), and

720 Thomas R. Clandinin (Stanford). We are grateful for in situ advice from Richard Benton/Steeve

721 Cruchet and Margo Herre. We thank the Center for Sensory Biology Imaging Facility (NIH

722 P30DC005211) for use of the LSM700 confocal microscope, and the Johns Hopkins School of

723 Public Health Malaria Research Institute for use of the Olympus SZX7 microscope equipped

724 with QImaging QIClick Cooled digital CCD camera. We thank J. Raji, S. Maguire, and J.

725 Konopka for comments on the manuscript and members of the Potter and Menuz labs for

726 discussion. We thank the Vosshall lab for sharing their Aedes findings before publication. This

727 work was supported by a Shelanski award to C.-C. L; a Wellcome Trust Collaborative Award

728 (203261/Z/16/Z) and an NIH BRAIN Initiative grant (1RF1MH120679-01) to G.S.X.E. Jefferis;

729 grants from the National Institutes of Health to H.L. (R00 AG062746); grants from the National

730 Institutes of Health to K.M. (NIGMS R35GM133209; NIDCD 1R21DC017868); grants from the

731 Department of Defense to C.J.P. (W81XWH-17-PRMRP) and from the National Institutes of

732 Health to C.J.P. (NIAID R01AI137078; NIDCD R01DC013070).

733 Portions of this work appear in Chapter 3 of D.T.'s doctoral dissertation. 
734

735

736

737

738

739

740

741

742

743

744

745

746

747

748

749

750

751

752

753

754

755

756

\section{Author Contributions}

Conceptualization: D.T. (knock-in analysis, SSR, AL maps and glomerular analyses), C-C.L. (HACK development, knock-in analysis, SSR, optogenetics), A.V. (SSR), M.B. (snRNAseq), P.S. (EM reconstruction and analyses), H.L. (snRNAseq), K.M. (SSR, IRs, palp analysis), C.J.P. (all aspects); Methodology: D.T. (cloning and generation of knock-ins (Ir25a, Ir8a, Ir76b), immunohistochemistry, SSR, statistics, AL maps), C-C.L. (HACK, genetics, cloning and generation of knock-ins (Orco, Ir8a, Ir76b), cloning and generation of QUAS-CsChrimson, SSR, optogenetics), A.V. (SSR), A.A. (SSR), S.B. (immunohistochemistry), M.B. (snRNAseq), P.S. (EM reconstruction and analyses), H.L. (snRNAseq), K.M. (SSR, IR expression), C.J.P. (all aspects); Formal Analysis: D.T. (knock-ins, SSR, cell counts), C-C.L. (knock-ins, SSR), A.V. (SSR), A.A. (SSR), S.B. (cell counts), M.B. (snRNAseq), P.S. (EM analyses), H.L. (snRNAseq); Investigation: D.T. (knock-in generation and validation (Ir25a, Ir8a, Ir76b), immunohistochemistry, AL analyses), C-C.L. (HACK development, knock-in generation and validation (Orco, Ir8a, Ir76b), QUAS-CsChrimson generation and validation, optogenetics, SSR - palp, ab2), A.V. (SSR - coeloconic), A.A. (SSR - ab3), S.B. (immunohistochemistry), M.B. (snRNAseq), P.S. (EM reconstruction and analyses), H.L. (snRNAseq); Writing, Original Draft: D.T. and C.J.P.; Writing, Review \& Editing: All Authors; Visualization: D.T. (olfactory system/fly cartoons, AL maps, microscopy images, SSR, Venn diagram, tables), C-C.L. (genetics cartoons, crossing schemes, SSR, optogenetics), M.B. (snRNAseq), P.S. (EM traces and dendrograms), H.L. (snRNAseq), C.J.P. (all aspects); Supervision: G.S.X.E.J., K.M. and C.J.P.; Funding Acquisition: C.J.P.

\section{Declaration of Interests}

The authors declare no competing interests. 


\section{Figures and Tables}

\section{$758 \quad$ Figure 1}

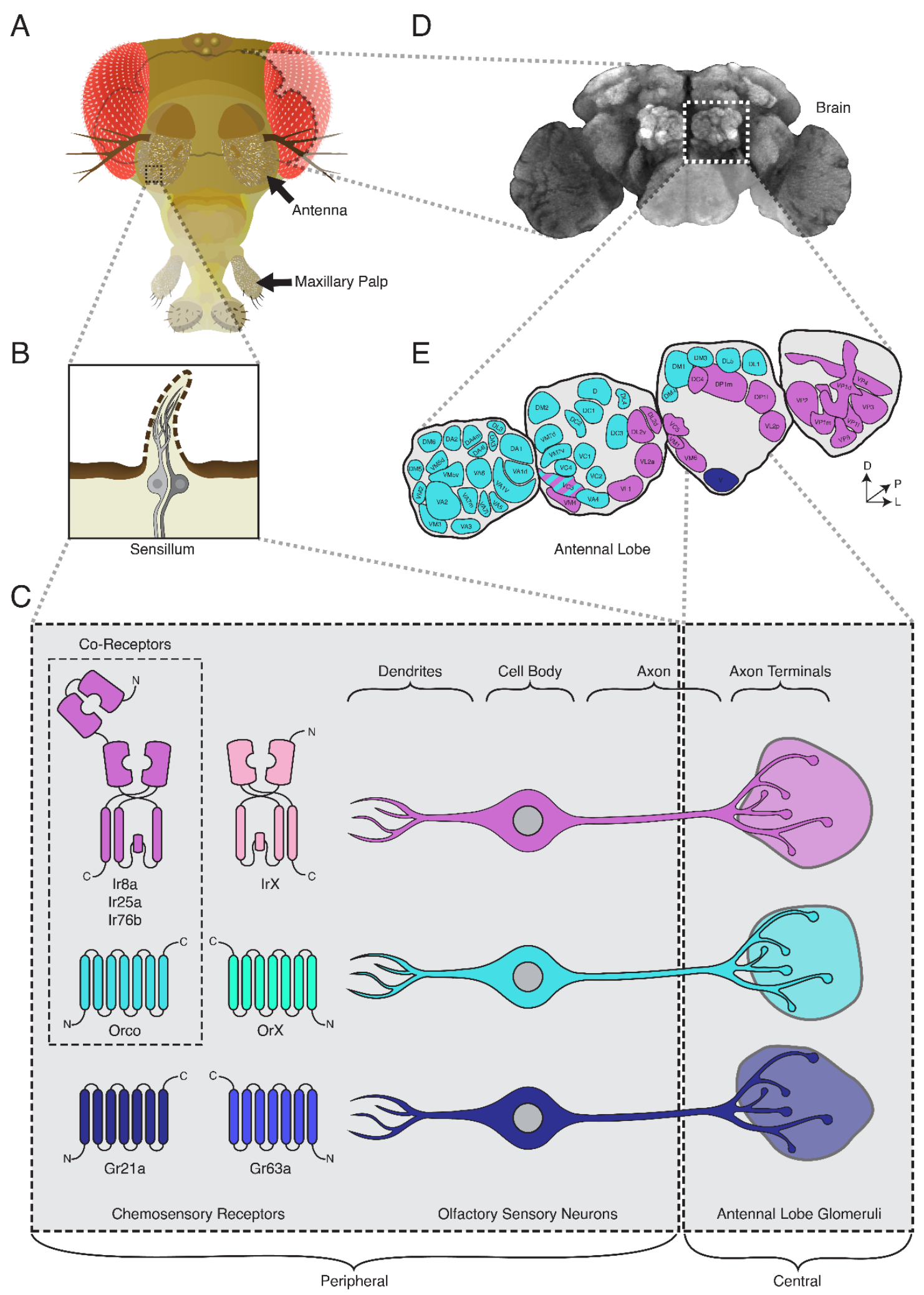



melanogaster.

A. The adult fly head (left) has two olfactory organs: the antennae and the maxillary palps

762 (arrows). Olfactory neurons from these organs project to the fly brain (D), to the first center

763 involved in processing of olfactory information, the antennal lobes (E). B. The olfactory organs

764 are covered by sensory hairs called sensilla (left). Each sensillum contains between one and

765 four olfactory sensory neurons (two example neurons are shown in grey). The dendrites of

766 these neurons extend into the sensilla, and the axons target discrete regions of the antennal

767 lobes called glomeruli (E). Neuronal compartments (dendrites, cell body, axon, axon terminals)

768 are labeled in (C). C. Left: in the periphery, each olfactory sensory neuron is traditionally thought

769 to express chemosensory receptors from only one of three gene families on its dendrites:

770 Ionotropic Receptors (IRs, pink and purple), Odorant Receptors (ORs, teal and green), or

771 Gustatory Receptors (GRs, light and dark blue). IRs and ORs require obligate co-receptors

772 (dotted box outline) to form functional ion channels. All ORs utilize a single co-receptor, Orco

773 (teal), while IRs can utilize one (or a combination) of three possible co-receptors (purple): Ir8a,

774 Ir25a, or Ir76b. The two GRs form a functional carbon dioxide detecting channel expressed in

775 only one class of neurons. All other olfactory neurons express one of the four co-receptors.

776 Right: olfactory sensory neurons expressing ORs, IRs, and GRs are thought to project to

777 mutually exclusive glomeruli in the $\mathrm{AL}$ of the central brain, forming the olfactory map shown in

778 (E). D. Fly brain stained with anti-brp synaptic marker (nc82), with left antennal lobe (AL)

779 outlined by dotted white box. E. AL map with glomeruli color-coded by the chemosensory

780 receptors (ORs, IRs, or GRs) expressed in the olfactory sensory neurons projecting to them.

781 Only one glomerulus (VC3, striped) receives inputs from neurons expressing chemoreceptors

782 from multiple gene families (ORs and IRs). Compass: $D=$ dorsal, $L=$ lateral, $P=$ posterior. 


\section{Figure 2}

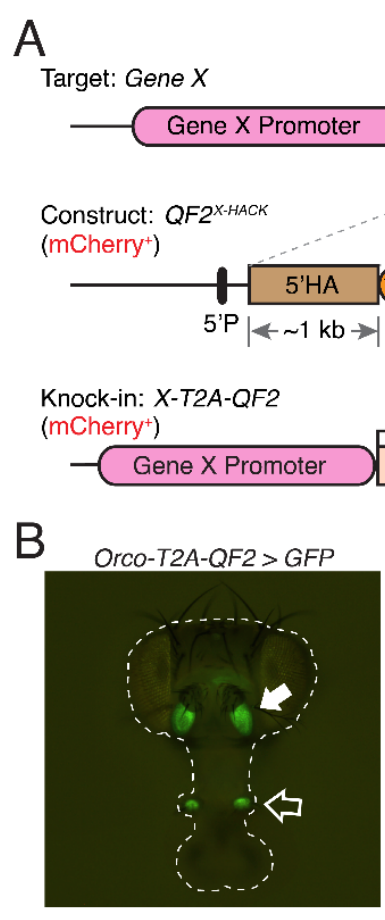

D

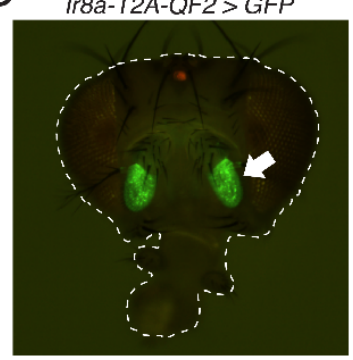

$\mathrm{F}$

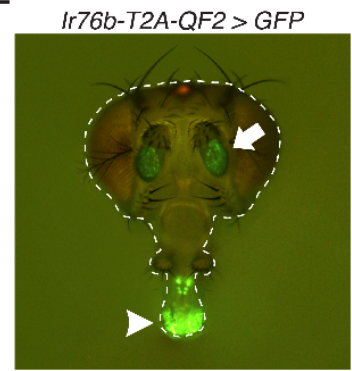

$\mathrm{H}$

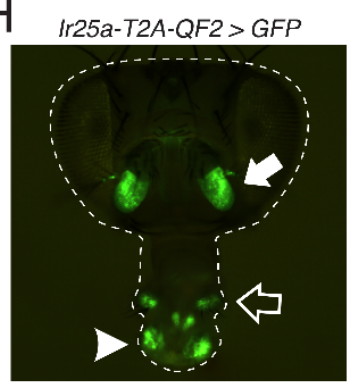

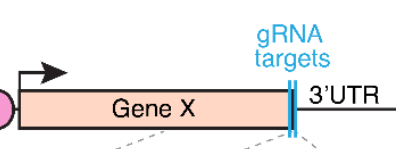
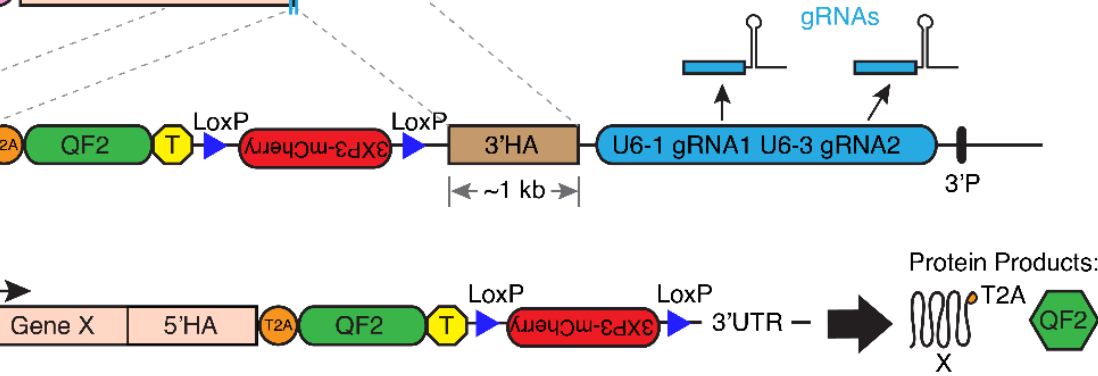

C

Orco-T2A-QF2 > GFP

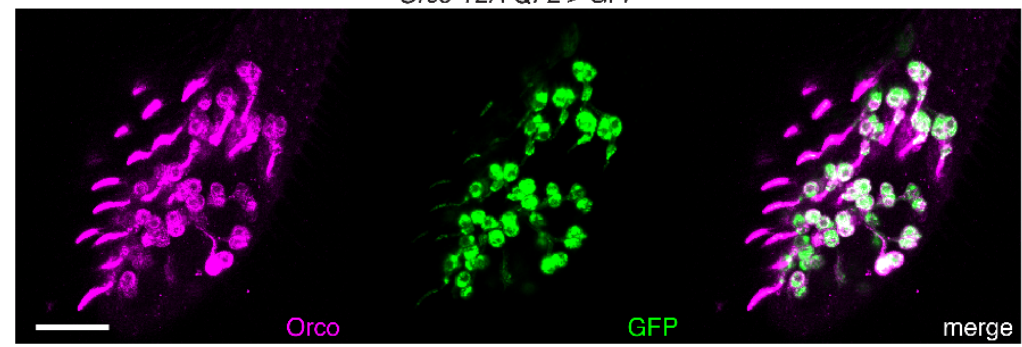

$\mathrm{E}$

Ir $8 \mathrm{a}-T 2 A-Q F 2>$ GFP

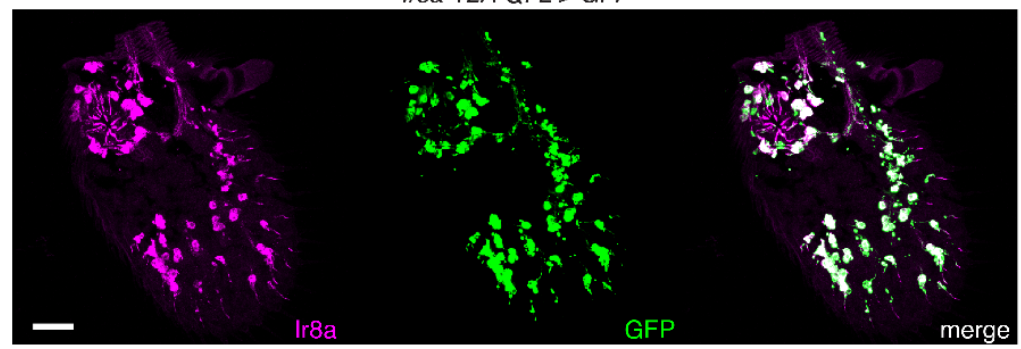

G

Ir76b-T2A-QF2 > GFP

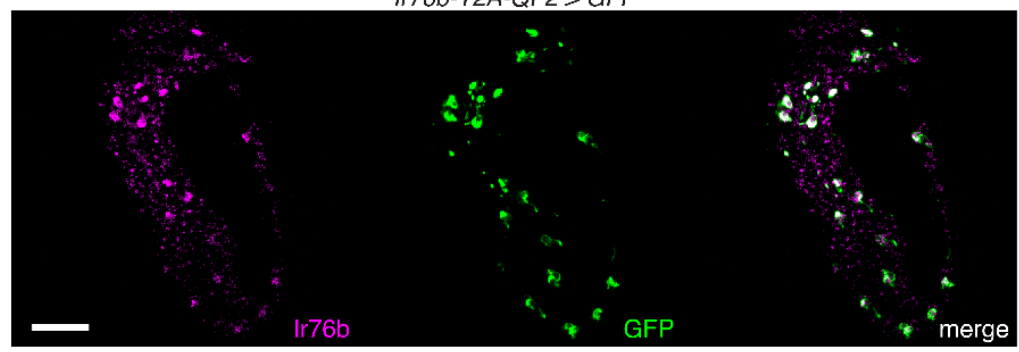

I

Ir25a-T2A-QF2 > GFP

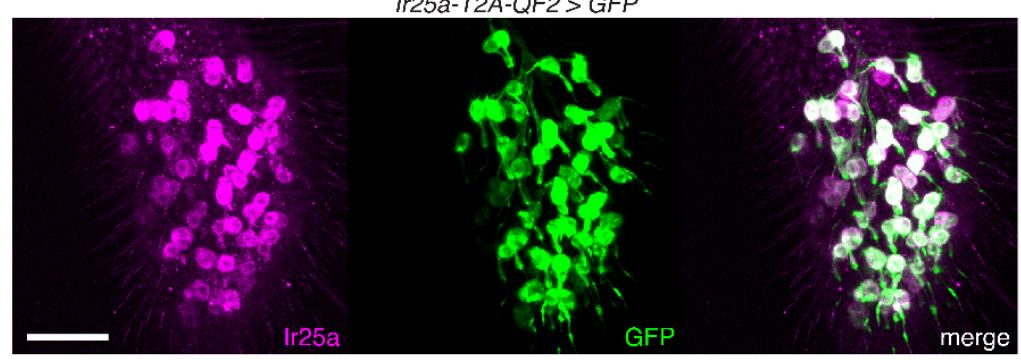


Figure 2. Generation and Validation of Chemosensory Co-Receptor Knock-in Lines.

785

786

787

788

789

790

791

792

793

794

795

796

797

798

799

800

801

802

803

804

805

806

807

808

809

A. Schematic of HACK knock-in approach. Top: two double-stranded breaks are induced on either side of the target gene stop codon with gRNAs (blue) expressed from the $Q F 2^{X-H A C K}$ construct (middle) in the presence of Cas9. The construct includes T2A-QF2 and a floxed 3XP3mCherry marker. The knock-in introduces a transcriptional stop (yellow T) after QF2. Bottom: the knock-in produces two protein products (right) from the targeted mRNA: target $\mathrm{X}$ and the QF2 transcription factor (Diao and White, 2012). The $X-T 2 A-Q F 2$ knock-in can be crossed to a reporter (e.g. QUAS-GFP) to examine the endogenous expression pattern of the target gene. B. Orco-T2A-QF2 driving QUAS-GFP in adult fly head. GFP expression is found in the antennae (filled arrow) and maxillary palps (hollow arrow), as previously reported (Larsson et al., 2004). C. Whole-mount anti-Orco antibody staining in Orco-T2A-QF2>GFP maxillary palps reveals a high degree of overlap of Orco+ and GFP+ cells. $N=3$. D. Ir8a-T2A-QF2 drives GFP in the antennae (arrow), as previously reported (Abuin et al., 2011). E. Anti-Ir8a antibody staining of Ir8a-T2A-QF2> GFP antennal cryosections shows high correspondence between Ir8a+ and GFP+ cells. $\mathrm{N}=7$. F. Ir76b-T2A-QF2 drives GFP expression in the antennae (filled arrow) and labella (hollow arrow), reflecting Ir76b's role in olfaction and gustation, respectively (Benton et al., 2009; Zhang et al., 2013). G. In situs on Ir76b-T2A-QF2> GFP antennal cryosections to validate that the knock-in faithfully recapitulates the endogenous expression pattern. $\mathrm{N}=3 . \mathbf{H}$. Ir25a-T2A-QF2 drives GFP in the antennae (filled arrow) and labella (hollow arrow), which has been reported previously (Benton et al., 2009; Croset et al., 2010). Expression in the maxillary palps (arrowhead) has not been previously reported. I. Whole-mount maxillary palp staining with an anti-Ir25a antibody in Ir25a-T2A-QF2>GFP flies. The knock-in and Ir25a antibody co-labeled the majority of olfactory neurons in the palps. $N=5$. Scale bars $=25 \mu \mathrm{m}$. In $(D)$ and $(F)$, the 3XP3-mCherry knock-in marker can be weakly detected in the eyes and ocelli (red spot) of both Ir8a-T2A-QF2 and Ir76b-T2A-QF2. See also Figure 2-Figure Supplements 1-4, Tables 1 and 2, Table 1-Source Data 1, Figure 2-Source Data 1, and Methods. 
Figure 3

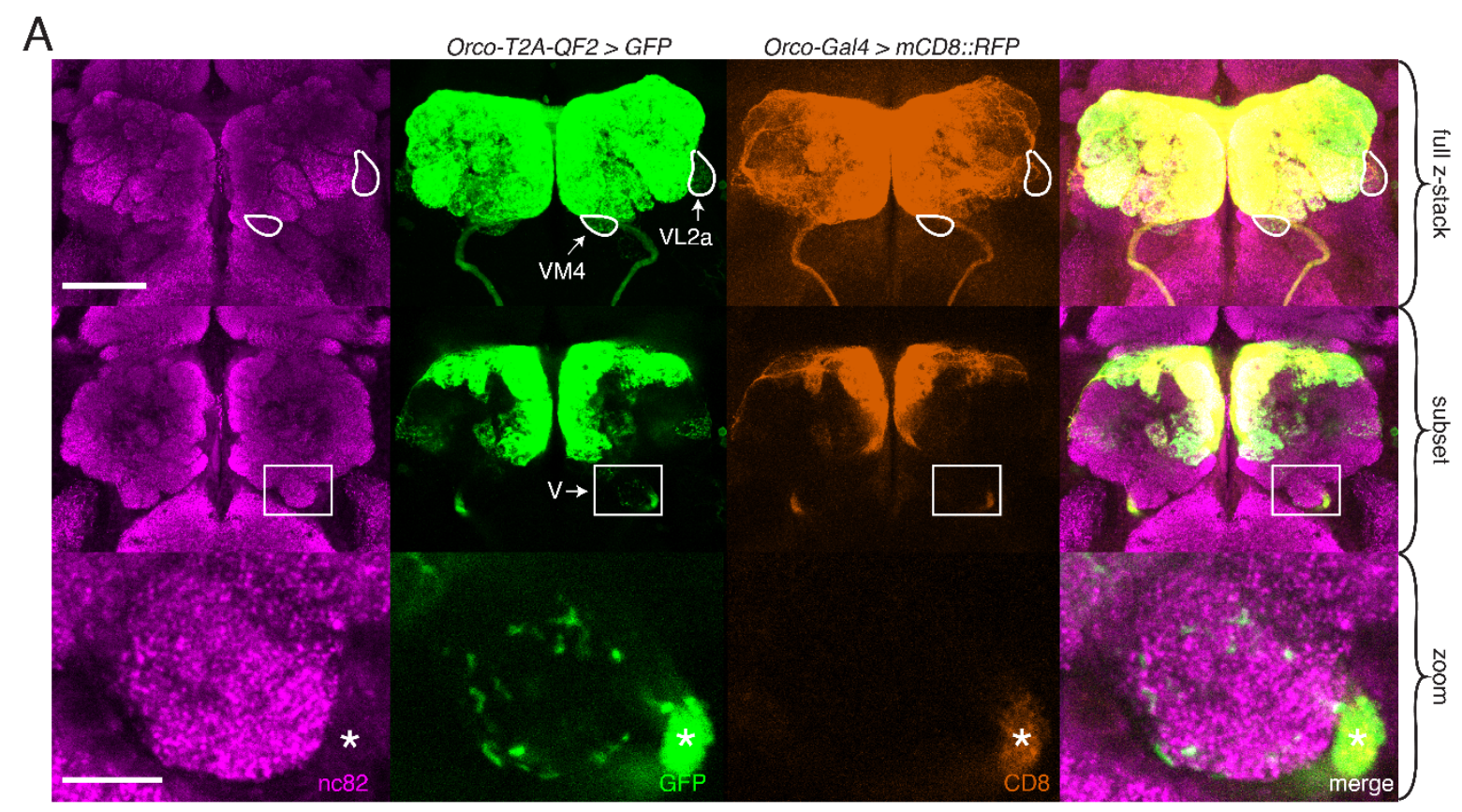

B

Ir8a-T2A-QF2 > GFP

$|r 8 a-G a| 4>m C D 8:: R F P$

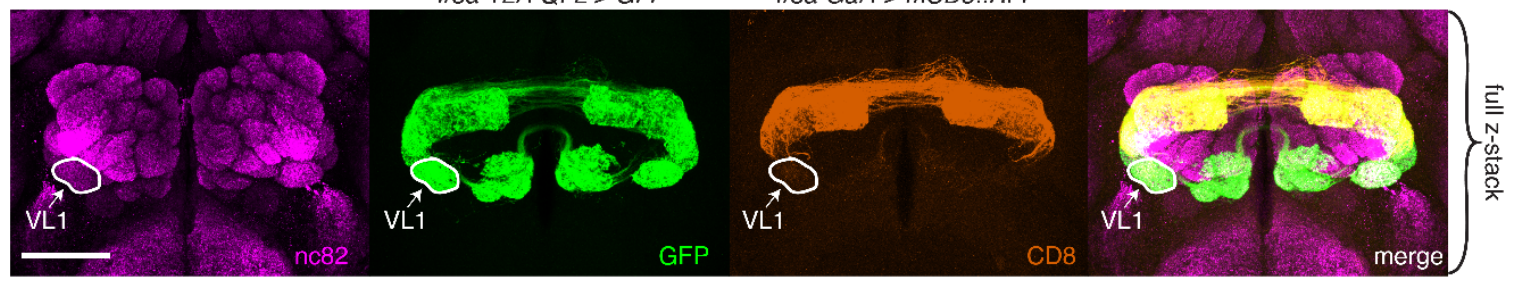

C

Ir76b-T2A-QF2 > GFP

Ir76b-Gal4 > mCD8::RFP

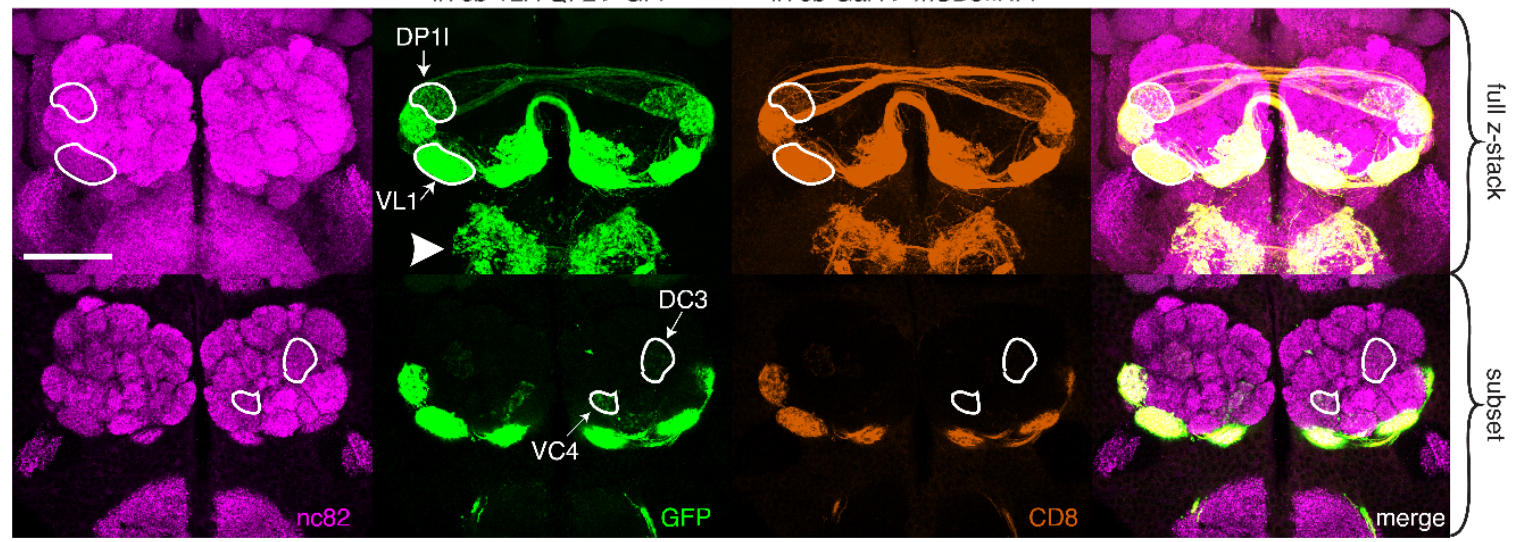

D

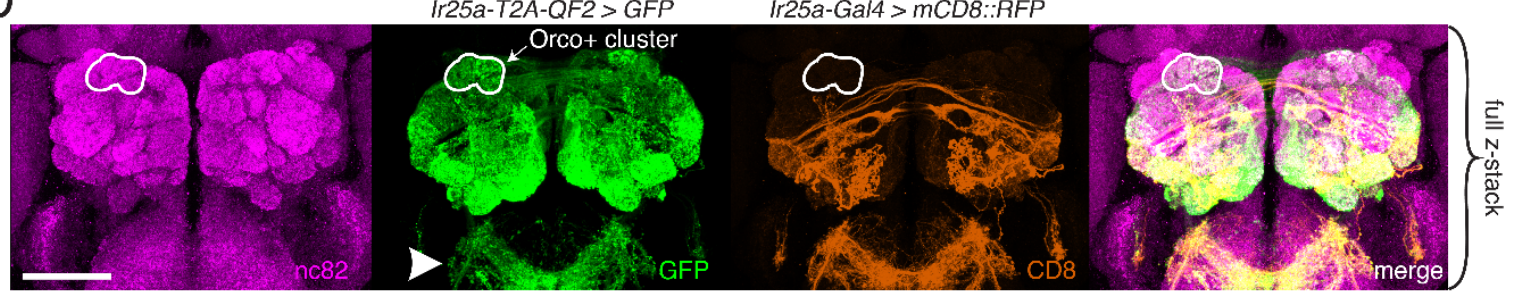


811 Figure 3. Expanded Expression of Olfactory Co-Receptors.

812 A-D. Comparing knock-in innervation patterns of the AL with what has previously been reported

813 for each co-receptor. Co-labeling experiments with each co-receptor knock-in line driving

814 QUAS-GFP (green) and the corresponding transgenic co-receptor Gal4 line driving UAS-

$815 m C D 8:: R F P$ (anti-CD8, orange). The nc82 antibody labels synapses (magenta) and is used as a

816 brain counterstain in these and all subsequent brain images. A. The Orco-T2A-QF2 knock-in

817 labels more glomeruli than the Orco-Gal4 line. Top: maximum intensity projection of full z-stack

818 showing two additional glomeruli labeled by the knock-in, VM4 (Ir8a+/Ir76b+/Ir25a+) and VL2a

819 (Ir8a+). Middle: subset of z-stack with a box around the $\mathrm{V}$ glomerulus. Bottom: zoom of boxed

820 region showing sparse innervation of the $\mathrm{V}$ glomerulus $(\mathrm{Gr} 21 \mathrm{a}+/ \mathrm{Gr} 63 \mathrm{a}+)$ by the knock-in but not

821 the Gal4 line. Asterisk indicates antennal nerve which is outside the $\mathrm{V}$ glomerulus. In the sub z-

822 stack and zoom panel, gain has been increased in the GFP channel to visualize weak labeling

823 more clearly. B. The Ir8a-T2A-QF2 knock-in also drives GFP expression in more glomeruli than

824 previously reported, including the outlined VL1 glomerulus (Ir25a+). C. In the brain, Ir76b-T2A-

825 QF2 > GFP olfactory neurons innervate the antennal lobes $(A L)$, while gustatory neurons from

826 the labella innervate the subesophageal zone (SEZ, arrowhead). Top: Both the Ir76b knock-in

827 and transgenic Gal4 line label more glomeruli than previously reported, including VL1 (Ir25a+)

828 and DP1I (Ir8a+). Bottom: The Ir76b-T2A-QF2 knock-in labels several Orco+ glomeruli, such as

829 DC3 and VC4 (outlined). In the subset, gain has been increased in the GFP channel to visualize

830 weakly labeled glomeruli more clearly. D. The Ir25a-T2A-QF2 knock-in drives GFP expression

831 broadly in the antennal lobes and SEZ (arrowhead). Ir25a+ neurons innervate many Orco+

832 glomeruli, such as those outlined. The transgenic Ir25a-Gal4 line labels a subset of the knock-in

833 expression pattern. $\mathrm{N}=3-10$ for co-labeling experiments, $\mathrm{N}=5-15$ for additional analyses of

834 the knock-in lines alone. Scale bars $=25 \mu \mathrm{m}$, except zoom panel scale bar $=10 \mu \mathrm{m}$. See also

835 Figure 3-Figure Supplements 1 and 2, Table 3, and Figure 3-Source data 1 and 2. 
Figure 4

A
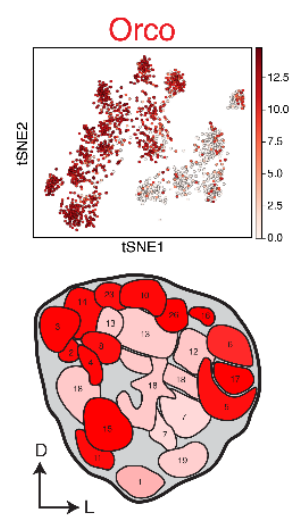

B

antenna

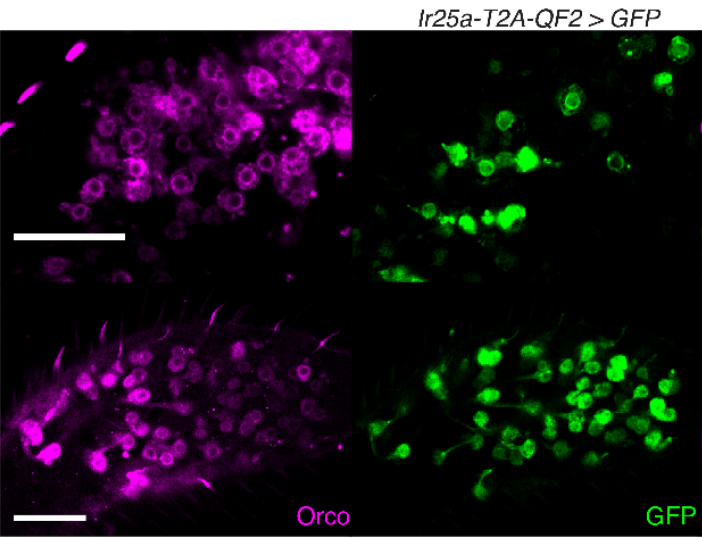

C

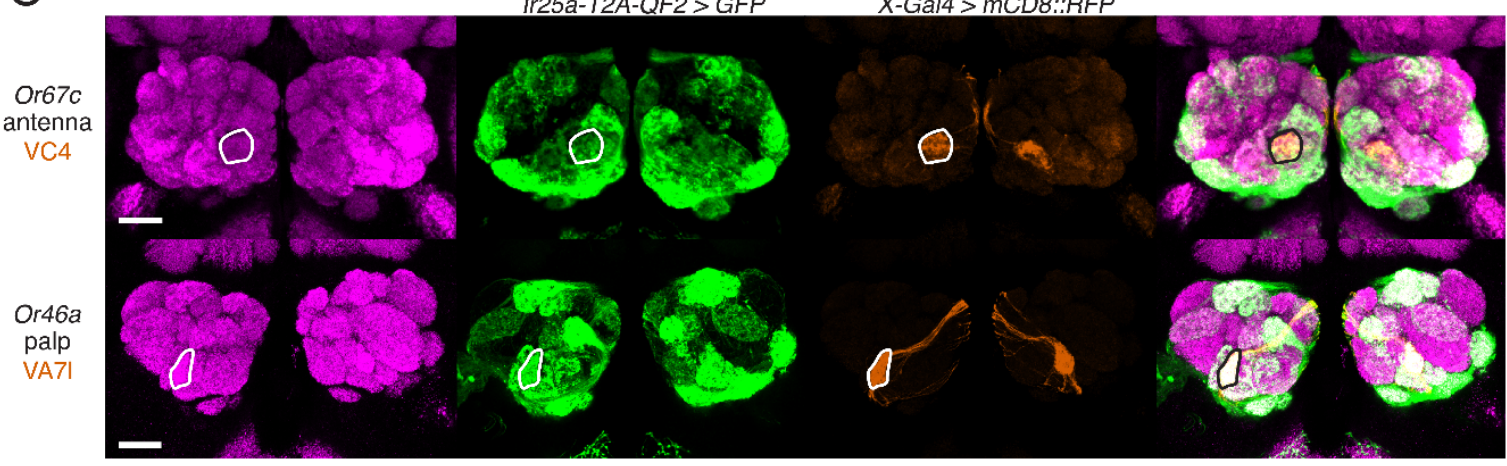

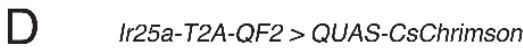

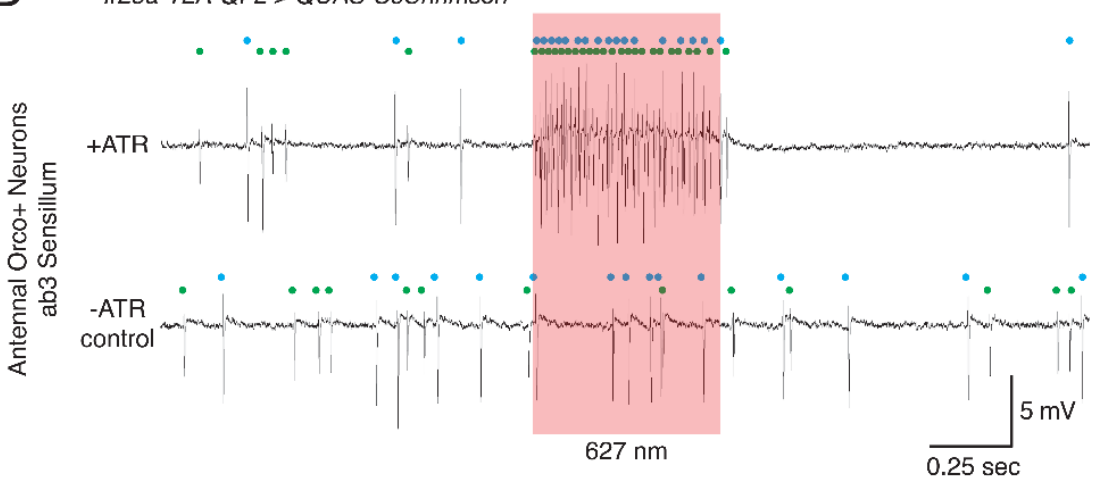

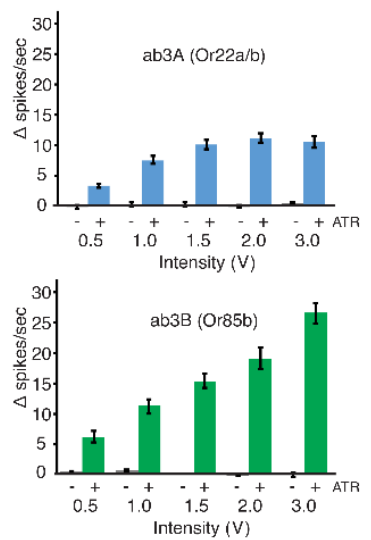


837 Figure 4. Confirmation of Co-Receptor Co-Expression.

838 A. snRNAseq of adult fly antennae (McLaughlin et al., 2021) confirms expanded expression of 839 olfactory co-receptors. Top: tSNE plots show expression of each co-receptor in 20 decoded 840 OSN clusters. Bottom: clusters were mapped to 24 glomeruli. Opacity of fill in each glomerulus 841 indicates the proportion of cells in that cluster expressing the given co-receptor, normalized to 842 total expression for that co-receptor gene (see Figure 4-Source Data 1). Right column: clusters 843 color-coded according to original chemoreceptor gene family. Compass: $D=$ dorsal, $L=$ lateral.

844 B. Anti-Orco antibody staining in antennal cryosections (top) and whole-mount palps (bottom)

845 confirms co-expression of Orco and Ir25a in the periphery (genotype: Ir25a-T2A-QF2 > GFP).

846 Right panels show cells pseudo-colored grey with specific single- or double-labeled cells

847 indicated by colored cell markers (GFP+ only in blue, GFP+ Orco+ in orange, Orco+ only in

848 red). C. Co-labeling experiments with various transgenic Gal4 lines driving mCD8::RFP (orange)

849 and the Ir25a-T2A-QF2 knock-in driving GFP (green). Ir25a-T2A-QF2 labels glomeruli

850 innervated by both antennal (top) and palpal (bottom) OSNs. D. Verification of Ir25a expression

851 in antennal ab3 sensilla using optogenetics. SSR recordings from ab3 Orco+ neurons in Ir25a-

852 T2A-QF2 > QUAS-CsChrimson flies. Representative traces from ab3 using 1.5V of $627 \mathrm{~nm}$ LED

853 light (red box) to activate CsChrimson. Bottom trace is control animal which was not fed the

854 required all-trans retinal cofactor (-ATR). Spikes from the ab3A and ab3B neurons are indicated

855 by blue and green dots, respectively. Right: Quantification of neuronal activity in response to

856 light at various LED intensities $(\mathrm{N}=7-12)$. These optogenetic experiments support Ir25a

857 expression in both ab3A neurons (Or22a/b, top; corresponding to DM2 glomerulus) and ab3B

858 neurons (Or85b, bottom; corresponding to VM5d glomerulus). Scale bars $=25 \mu \mathrm{m}$. See also

859 Figure 4-Figure Supplement 1, Table 3, and Figure 4-Source Data 1 - 3. 
Figure 5

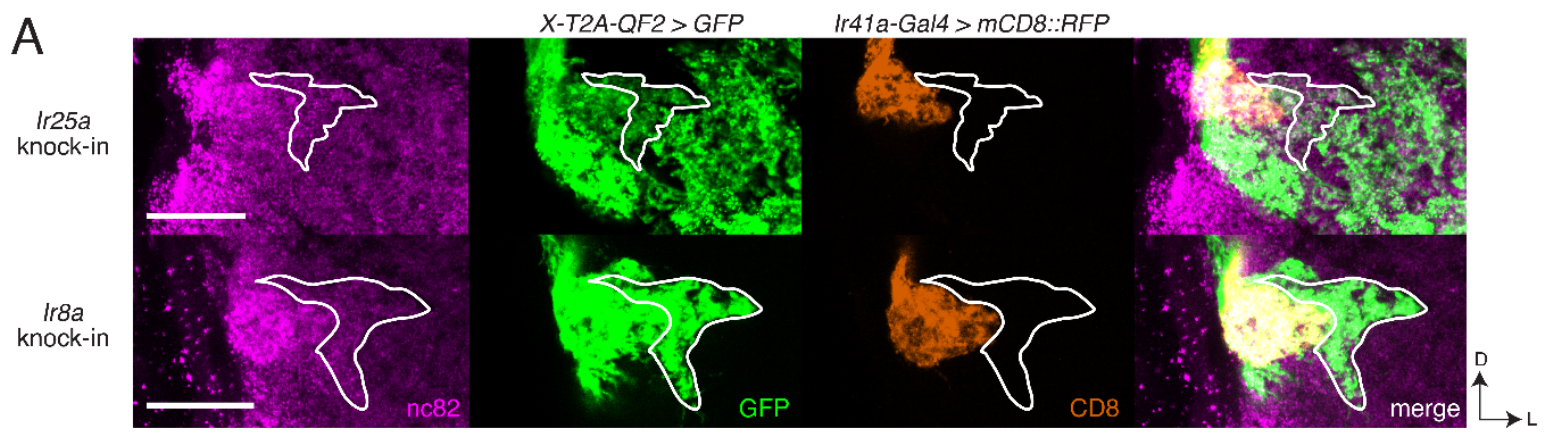

B

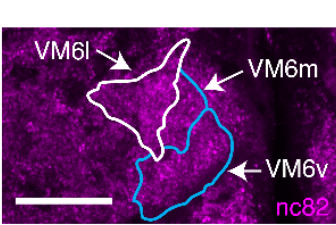

Ir8a-T2A-QF2 > GFP

Rh50-Gal4 > mCD8::RFP
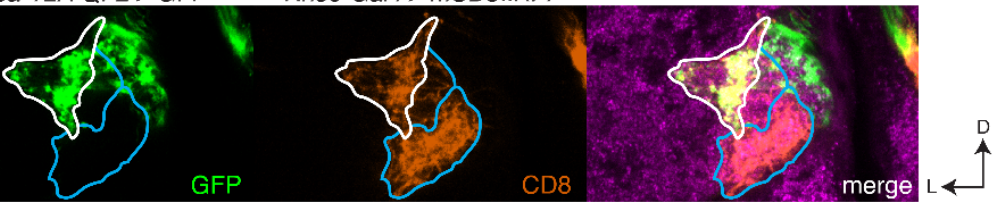

C

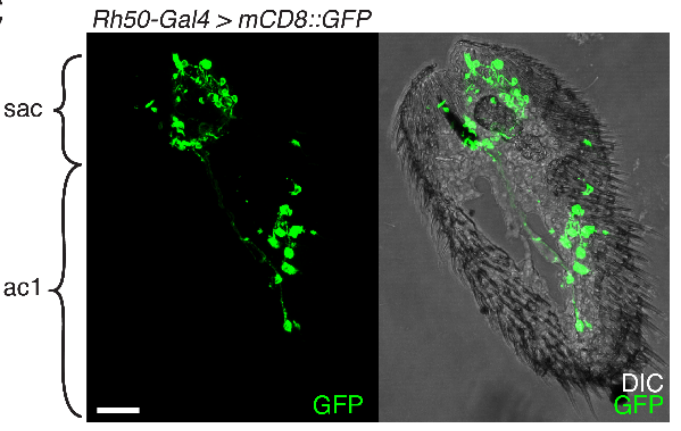

E

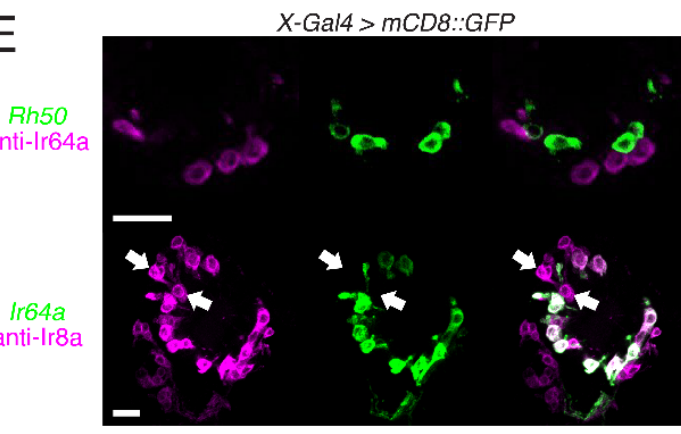

G

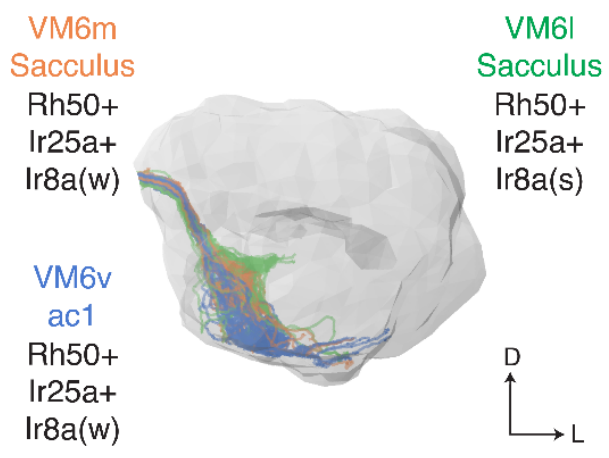

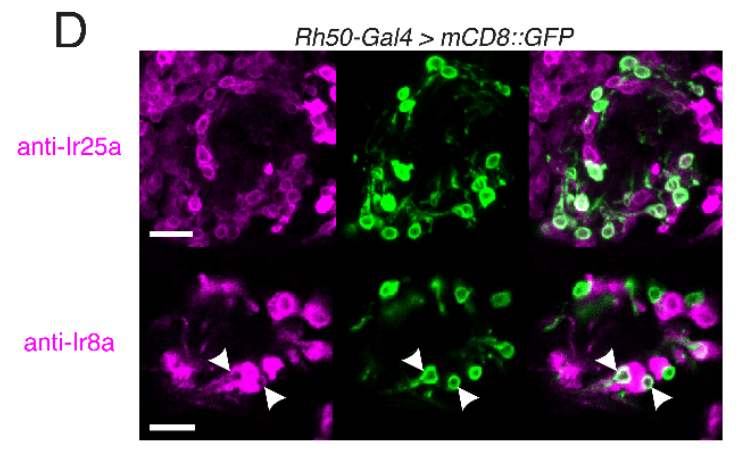

F

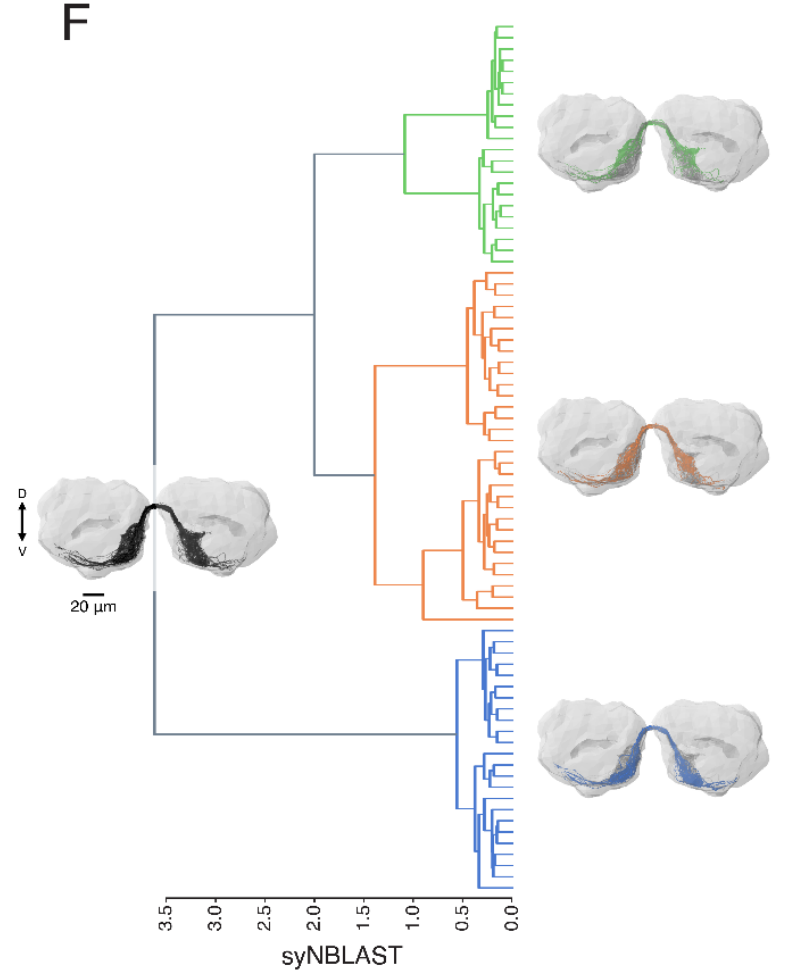


861 Figure 5. Identification of New OSN Classes.

862 A. Co-labeling experiments with Ir41a-Gal4 show that both Ir25a-T2A-QF2 and Ir8a-T2A-QF2

863 label the VC5 glomerulus (orange), and also a previously unidentified AL region (outline). B.

864 The new innervation pattern corresponds to the 'horn' (white outline) of the VM6 glomerulus

865 labeled by Rh50+ neurons (orange). One portion of VM6 is strongly Ir8a+ (VM6I), while two

866 other portions show little to no Ir8a expression (VM6m and VM6v, blue outlines). C. Rh50-Gal4

$867>$ GFP labels neurons in the sacculus (sac) and antennal coeloconic ac1 sensilla. D. In the

868 sacculus, all Rh50+ neurons appear to be Ir25a+ (top), and a subset are Ir8a+ (bottom,

869 arrowheads). E. Top: Rh50+ neurons in the sacculus do not overlap with Ir64a+ neurons.

870 Bottom: there are two distinct populations of Ir8a+ neurons in the sacculus - those that are

871 Ir64a+ and those that are Ir64a- (arrows). The latter likely correspond to Rh50+ neurons. F. EM

872 reconstructions of VM6 OSNs in a full brain volume (Dorkenwald et al., 2020) reveal three

873 distinct subpopulations. G. Model of OSN innervation of the VM6 region. VM6 can be

874 subdivided into three OSN populations based on anatomical location in the periphery and

875 chemoreceptor expression: VM6v (blue) OSNs originate in ac1, strongly (s) express Rh50 and

876 Ir25a, and weakly (w) or infrequently express Ir8a; VM6m (orange) neurons originate in the

877 sacculus and have a similar chemoreceptor expression profile to VM6v; VM6I (green) OSNs

878 originate in the sacculus but strongly express Ir8a in addition to Rh50 and Ir25a. Compass: D = 879 dorsal, $L=$ lateral. Scale bars: $20 \mu \mathrm{m}$ in (A-C) and (F), $10 \mu \mathrm{m}$ in (D-E). See also Figure 5-Figure 880 Supplement 1 and Tables 3 and 4. 
bioRxiv preprint doi: https://doi.org/10.1101/2020.11.07.355651; this version posted July 27,2021 . The copyright holder for this preprint (which was not certified by peer review) is the author/funder. All rights reserved. No reuse allowed without permission.

\section{$881 \quad$ Figure 6}

A

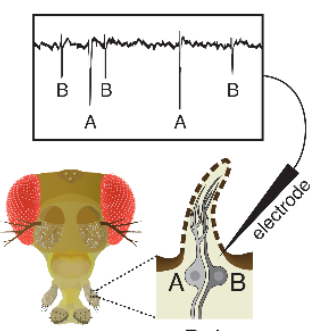

Palp

pbX Sensillum
B C

$1 \% 1$-octen-3-ol

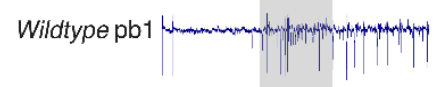

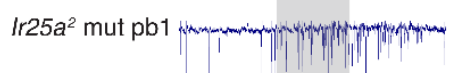

Orco $^{2}$ mut pbX

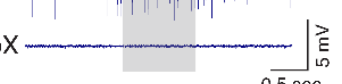

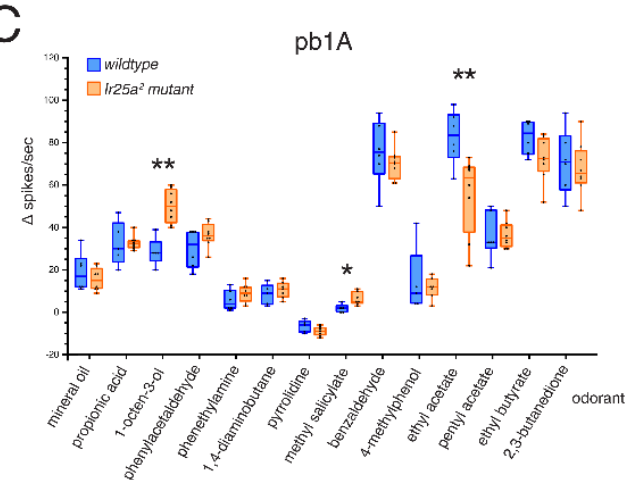

D

\begin{tabular}{|c|c|c|c|c|c|c|c|}
\hline \multicolumn{2}{|r|}{ Stimulus } & \multicolumn{6}{|c|}{ Neuron (Receptor) } \\
\hline Chemical Class & Odorant & $\begin{array}{c}\mathrm{pb} 1 \mathrm{~A} \\
\text { (Or42a) }\end{array}$ & $\begin{array}{l}\text { pb1B } \\
\text { (Or71a) }\end{array}$ & 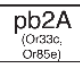 & $\begin{array}{l}\text { pb2B } \\
\text { (Or46a) }\end{array}$ & $\begin{array}{c}\text { pb3A } \\
\text { (Or59c) }\end{array}$ & $\begin{array}{c}\text { pb3B } \\
\text { (Or85d) }\end{array}$ \\
\hline acid & propionic acid & $\sum$ & $\sum$ & $\sum$ & $\sum$ & $\sum$ & $\sum$ \\
\hline alcohol & 1-octen-3-ol & & N.D. & $\sum<$ & & & $\sum<$ \\
\hline aldehyde & phenylacetaldehyde & $\sum<$ & & & $\sum<$ & $\sum<$ & \\
\hline amine & phenethylamine & $\sum<$ & & 25 & $\sum<$ & $\sum<$ & $\sum<$ \\
\hline amine & 1,4-diaminobutane (putrescine) & $\sum$ & $\sum$ & . & $\sum<$ & $\sum$ & $\sum$ \\
\hline amine & pyrrolidine & & & $><$ & & $\sum$ & \\
\hline aromatic & methyl salicylate & & & $\sum$ & $\sum$ & & $\sum$ \\
\hline aromatic & benzaldehyde & & N.D. & $?$ & $?$ & $\sum<$ & \\
\hline aromatic & 4-methylphenol ( $\mathrm{p}$-cresol) & $\sum 3$ & & & & $\sum<$ & $\sum$ \\
\hline ester & ethyl acetate & & N.D. & & $\sum<$ & $3<$ & $\sum<$ \\
\hline ester & pentyl acetate & 2 & $\sum$ & & & & \\
\hline ester & ethyl butyrate & & N.D. & & & & $\sum$ \\
\hline ketone & 2,3-butanedione & & N.D. & & & & \\
\hline
\end{tabular}

Higher in wildtype

Higher in 1 r $25 a^{2}$ mutant

No difference

No response

N.D. No data

? May be $\mathrm{A}$ or $\mathrm{B}$ neuron

E

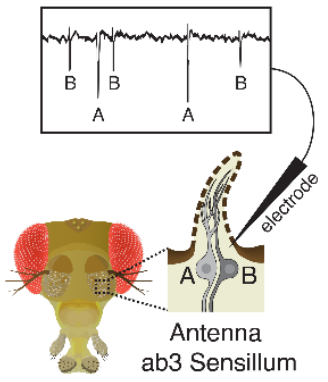

F

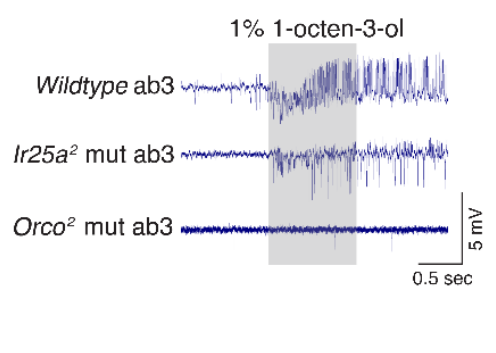

$\mathrm{H}$

\begin{tabular}{|c|c|c|c|c|}
\hline \multicolumn{3}{|c|}{ Stimulus } & \multicolumn{2}{|c|}{ Neuron (Receptor) } \\
\hline Chemical Class & Odorant & & $\begin{array}{c}\text { ab3A } \\
\text { (Or22a/b) }\end{array}$ & $\begin{array}{l}\text { ab3B } \\
\text { (Or85b) }\end{array}$ \\
\hline acid & propionic acid & & & \\
\hline alcohol & 1-octen-3-ol & & & \\
\hline aldehyde & phenylacetaldehyde & & & \\
\hline amine & phenethylamine & & $3<$ & $3<$ \\
\hline amine & 1,4-diaminobutane (putresc & cine) & $3<$ & $3<$ \\
\hline amine & pyrrolidine & & & \\
\hline aromatic & methyl salicylate & & $3<$ & $3<$ \\
\hline aromatic & benzaldehyde & & $3<$ & $2<$ \\
\hline aromatic & 4-methylphenol (p-creso & & 3 & $3<$ \\
\hline ester & ethyl acetate & & & $3<$ \\
\hline ester & pentyl acetate & & & \\
\hline ester & ethyl butyrate & & & \\
\hline ketone & 2,3-butanedione & & & \\
\hline
\end{tabular}

G

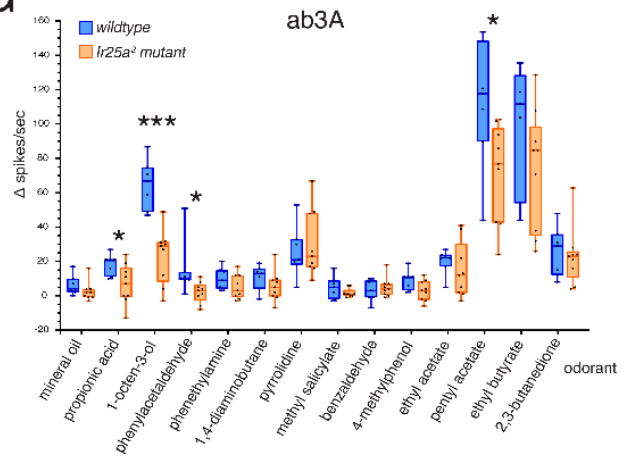

I

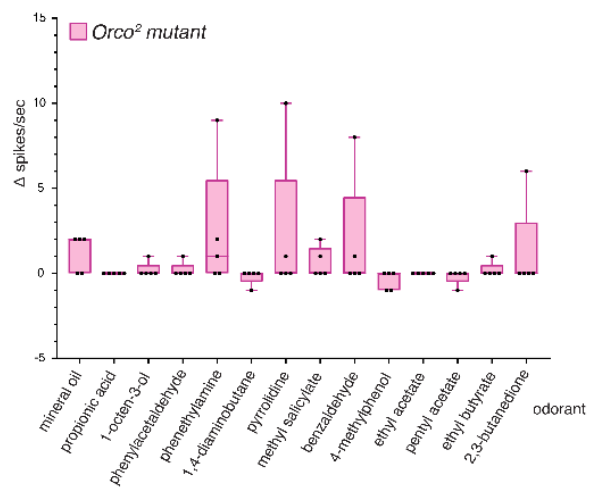


Figure 6. Co-Receptor Contributions to Olfactory Neuron Physiology.

A-I. SSR experiments were performed in three genetic backgrounds: wildtype, $\operatorname{Ir} 25 \mathrm{a}^{2}$ mutant, and $\operatorname{Orco}^{2}$ mutant flies. A panel of 13 odorants was tested. In all box plots, ${ }^{*} p<0.05,{ }^{* *} p<$ 0.01 , and ${ }^{* * *} p<0.001$. A. Cartoon of a fly head, zooming in on a single sensillum in the palp. Each palpal sensillum ( $\mathrm{pbX}$ ) contains two neurons, A and B. An electrode is inserted into the sensillum, and neuronal activity is recorded in response to odorants. Activity of the A and B neurons can be distinguished based on their spike amplitudes (top). B. Representative traces from recordings in palp basiconic pb1 sensilla in the three genotypes in response to $1 \% 1$ octen-3-ol. Sensilla were identified based on responses to reference odorants (de Bruyne et al., 1999). The $\operatorname{Orco}^{2}$ mutant did not exhibit odor-evoked activity nor spontaneous activity making it difficult to determine the identity of the recorded sensillum. $\mathrm{Orco}^{2}$ mutant sensilla are thus denoted pbX. C. Quantification of responses to the panel of odorants in wildtype (blue; $N=5-9$ flies) and $I r 25 a^{2}$ mutant (orange; $N=6-10$ flies) pb1A neurons. Responses were higher in the Ir25a $a^{2}$ mutant than in the wildtype for 1-octen-3-ol and methyl salicylate, and lower in the $/ r 25 a^{2}$ mutant for ethyl acetate. Mann-Whitney $U$ tests indicated these differences were statistically significant: 1-octen-3-ol: $M d n_{\text {Ir25amut }}=50, M d n_{\text {wildtype }}=28, U\left(N_{\text {Ir25amut }}=8, N_{\text {wildtype }}=5\right)=0, p=$ 0.0016; methyl salicylate: $M d n_{\text {Ir25amut }}=5, M d n_{\text {wildtype }}=2, U\left(N_{\text {Ir25amut }}=7, N_{\text {wildtype }}=5\right)=3, p=$ 0.0177; ethyl acetate: $M d n_{\text {Ir25amut }}=63.5, M d n_{\text {wildype }}=83.5, U\left(N_{\text {Ir25amut }}=8, N_{\text {wildtype }}=6\right)=4, p=$ 0.008. D. Summary of differences in responses across all six neuron classes in the palps between wildtype and $/ r 25 a^{2}$ mutant flies. Comparisons were made using Mann-Whitney $U$ tests. Orange indicates higher response in $/ r 25 a^{2}$ mutant, blue indicates higher response in wildtype. Grey is no difference between genotypes, $\mathrm{X}$ indicates no response to the given stimulus, and N.D. is no data (strong A neuron response obscured B neuron spikes preventing quantification). In the wildtype, for one sensillum-odorant combination (pb2 and benzaldehyde), it could not be distinguished if responses arose from the A or B neuron or both (indicated by a question mark). E. Fly head cartoon, zooming in on a single sensillum in the antenna. We 
recorded from antennal ab3 sensilla, each of which contains two neurons, A and $B$. As in the palps, responses from these neurons can be distinguished based upon their spike amplitude (top). F. Representative traces from recordings in antennal basiconic ab3 sensilla in the three genotypes in response to $1 \%$ 1-octen-3-ol. In $\mathrm{Orco}^{2}$ mutant ab3 sensilla spontaneous activity was observed, but there was no significant odor-evoked activity. Wildtype $\mathrm{N}=7$ sensilla from 5 flies; Ir25a ${ }^{2}$ mutant $\mathrm{N}=10$ sensilla from 5 flies. G. Quantification of responses in wildtype (blue; $\mathrm{N}=7$ ) and $/ r 25 \mathrm{a}^{2}$ mutant (orange; $\mathrm{N}=9$ ) ab3A neurons. Responses were significantly higher in wildtype compared to $I r 25 a^{2}$ mutant ab3A neurons for four odorants (Mann-Whitney $U$ results in parentheses; all $N_{\text {wildtype }}=7$ and $\left.N_{\text {Ir25amut }}=9\right)$ : propionic acid $\left(M d n_{\text {wildtype }}=21, M d n_{\text {Ir25amut }}=7, U=\right.$ 12.5, $p=0.0441) ; 1$-octen-3-ol $\left(M d n_{\text {wildtype }}=67, M d n_{\text {Ir25amut }}=29, U=1.5, p=0.0004\right)$; phenylacetaldehyde $\left(M d n_{\text {wildtype }}=10, M d n_{\text {Ir } 25 a m u t}=3, U=9, p=0.015\right)$; and pentyl acetate $\left(M d n_{\text {wildtype }}=118, M d n_{\text {Ir25amut }}=77, U=9, p=0.0164\right)$. Difference between wildtype and $/ r 25 a^{2}$ mutant to phenylacetaldehyde is significant even with the large wildtype outlier removed $(p=$ 0.0336). H. Summary of differences in responses in the two neuron classes in ab3 between wildtype and $I r 25 \mathrm{a}^{2}$ mutant flies. Comparisons were made using Mann-Whitney $U$ tests. Orange indicates higher response in $\operatorname{Ir} 25 \mathrm{a}^{2}$ mutant, blue indicates higher response in wildtype, grey is no difference between genotypes, and $\mathrm{X}$ is no response to the given stimulus. One $\operatorname{Ir} 25 \mathrm{a}^{2}$ mutant fly was excluded from analyses as it had high responses to the mineral oil control (40 $53 \Delta$ spikes/sec), not seen in any other animal of any genotype. I. Weak responses in Orco ${ }^{2}$ mutant flies to certain stimuli ( $\leq 10 \Delta$ spikes/sec) were occasionally detected. While there were some statistically significant differences from mineral oil control (pentyl acetate $p=0.0109$, propionic acid $p=0.0434$, ethyl acetate $p=0.0434,1,4$-diaminobutane $p=0.0109, p$-cresol $p=$ $0.0021)$, these were not deemed biologically significant due to very small $\Delta$ spike values relative to zero. For more details, see Methods. $\mathrm{N}=5$ flies. See also Figure 6-Figure Supplements 1 and 2, and Figure 6-Source Data 1 and 2. 


\section{$933 \quad$ Figure 7}
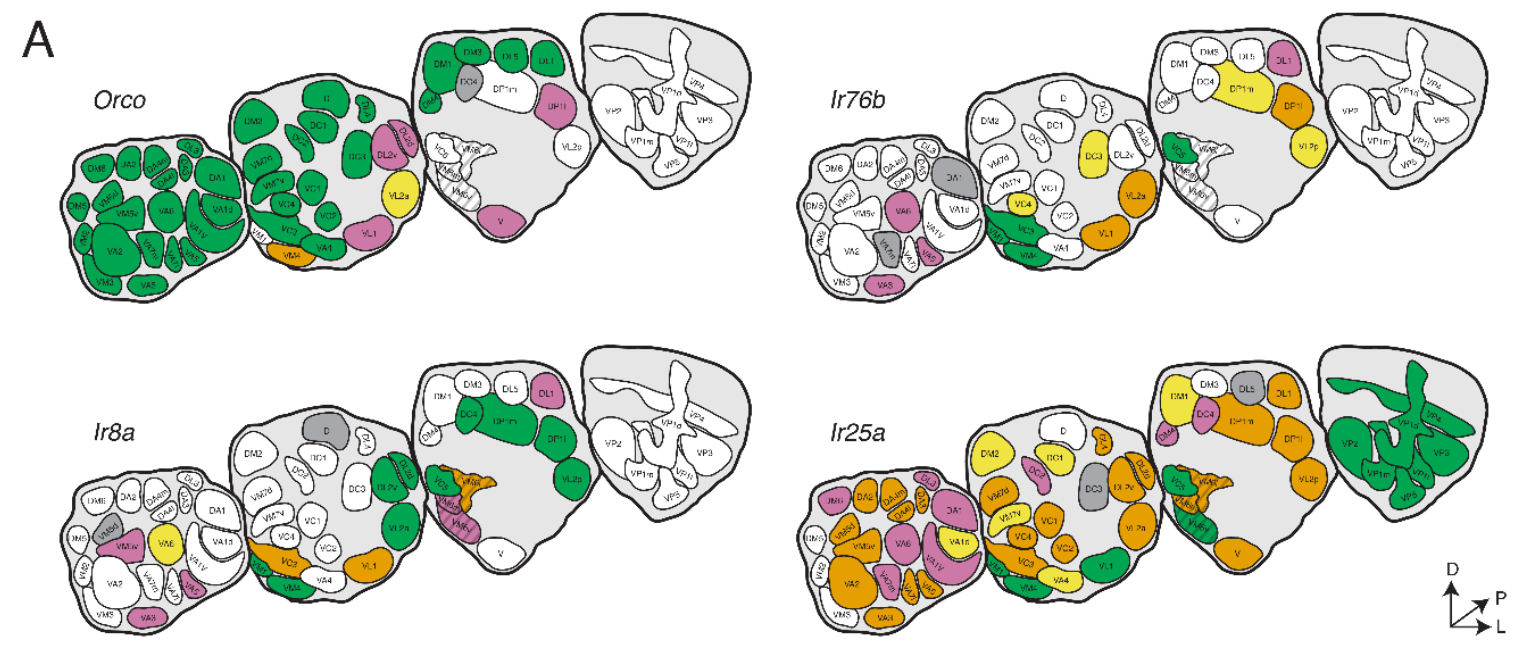

\begin{tabular}{|lll|}
\hline Previously reported & \multicolumn{2}{c|}{ New innervation: } \\
\hline No innervation & $\square$ Strong & $\square$ Weak \\
Intermediate & $\square$ Variable
\end{tabular}

B

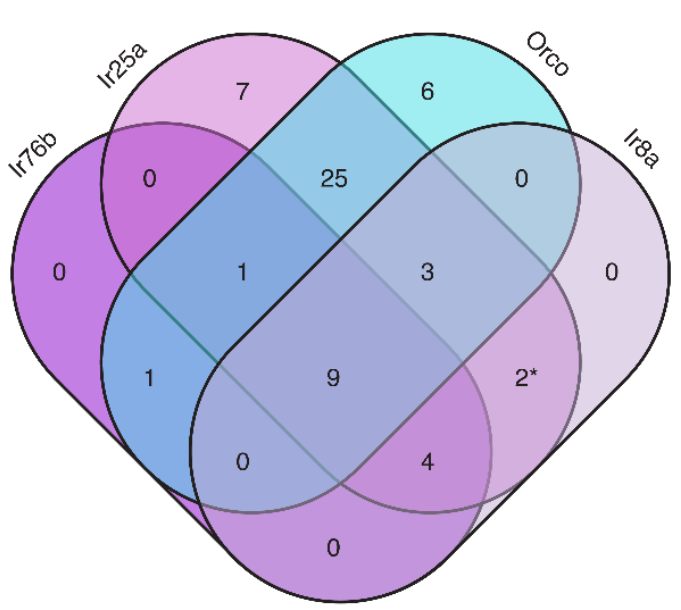

\begin{tabular}{|c|c|c|}
\hline Co-Receptor(s) & Total & Glomeruli \\
\hline Orco, Ir8a, Ir76b, Ir25a & 9 & DP11, VA3, VC3, VL1, VL2a, VM4, DL1, VA5, VA6 \\
\hline Ir8a, Ir76b, Ir25a & 4 & DP1m, VC5, VL2p, VM1 \\
\hline Orco, Ir8a, Ir25a & 3 & DL2d, DL2v, VM5v \\
\hline Orco, Ir76b, Ir25a & 1 & VC4 \\
\hline Orco, Ir25a & 25 & $\begin{array}{c}\text { DA1, DA2, DA3, DA41, DA4m, DC1, DC2, DL3, DL4, } \\
\text { DM1, DM2, DM4, DM6, V, VA1d, VA1v, VA2, VA4, } \\
\text { VA71, VA7m, VC1, VC2, VM5d, VM7d, VM77 }\end{array}$ \\
\hline Ir8a, Ir25a & $2^{*}$ & DC4, VM661, m, and v)* \\
\hline Orco, Ir76b & 1 & DC3 \\
\hline Ir25a & 7 & VP1d, VP11, VP1m, VP2, VP3, VP4, VP5 \\
\hline Orco & 6 & D, DL5, DM3, DM5, VM2, VM3 \\
\hline
\end{tabular}

C

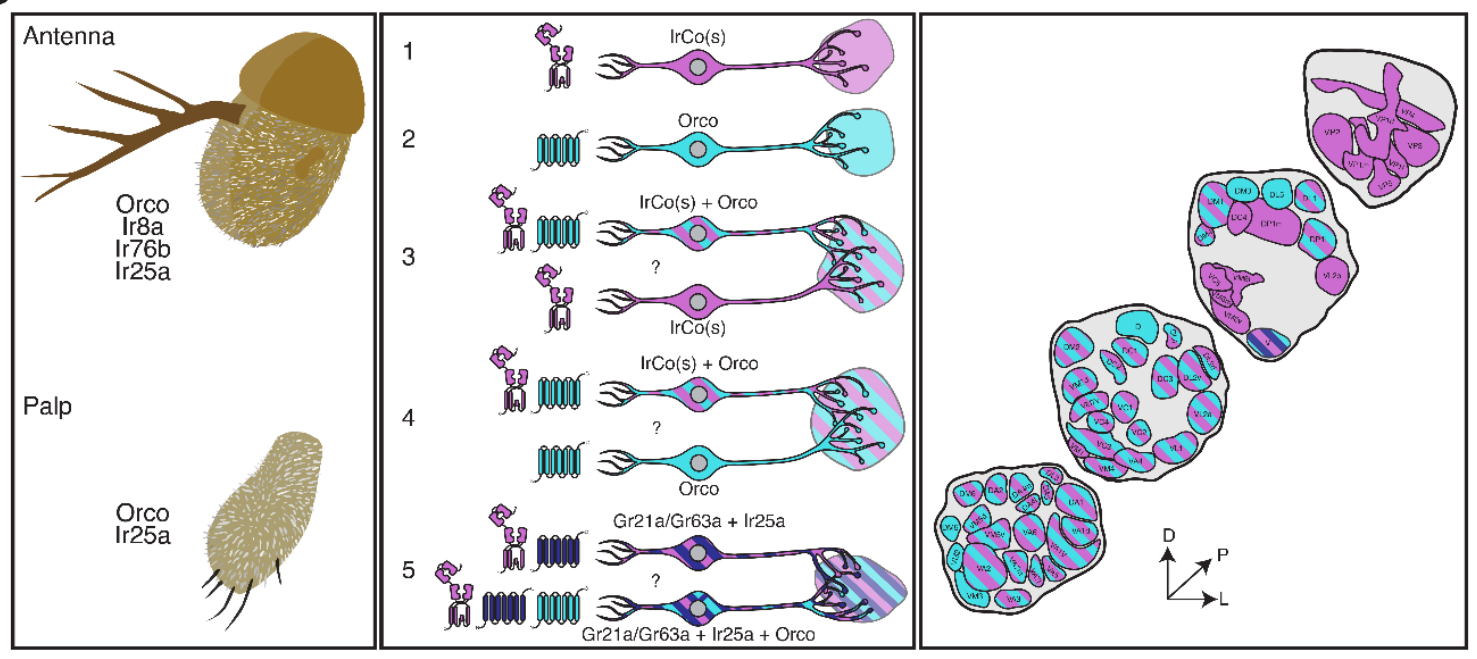


A. Summary of AL expression for all co-receptor knock-in lines (from all brains examined in Figure 3 - 5; Orco $\mathrm{N}=8, \operatorname{Ir} 8 a \mathrm{~N}=15, \operatorname{Ir} 76 b \mathrm{~N}=11, \operatorname{Ir} 25 a \mathrm{~N}=15)$. The previously reported innervation pattern for each co-receptor is shown in green; new innervation reported here is color-coded according to strength of glomerular labeling, from strong (orange), to intermediate (yellow), to weak (pink). Glomeruli labeled in $<50 \%$ of brains examined for a given knock-in line are designated variable (grey); glomeruli not labeled are white. The novel VM6 glomerular subdivisions reported here are indicated by grey stripes. B. Overlap of chemosensory modalities in the AL. In the Venn diagram (left), IR co-receptors are color-coded in shades of purple, while Orco is in teal, as in Figure 1. Numbers indicate how many glomeruli are found in the given intersection of co-receptors out of 58 total glomeruli. Variably labeled glomeruli were excluded from these analyses. The table lists the names of the glomeruli in each section of the Venn olfaction in Drosophila. Left: in the periphery, all four co-receptors are expressed in the antenna (top), while palpal neurons express Orco and Ir25a (bottom). Middle: Many different classes of OSNs express various combinations of chemosensory receptors and co-receptors. While some neurons express only IrCos (purple, \#1) or Orco (teal, \#2), many neurons co-express these chemoreceptors (indicated with striped fill, \#3 and 4). Within the latter group, there may be OSN populations in which IRs are the dominant receptors, and OR expression is sparse (\#3), and other populations where ORs are the primary receptors and IR expression is infrequent (\#4).

954 GR+ neurons (dark blue) also express Ir25a (\#5, dark blue and purple striped fill), and some of 955 these neurons additionally express $\operatorname{Orco}(\# 5$, dark blue, purple, and teal striped fill). Question 956 marks indicate potential instances of co-convergence of different sub-types of OSNs onto the 957 same glomeruli. Right: a comprehensive map of the antennal lobe shows that most glomeruli 958 are innervated by OSNs that co-express multiple chemoreceptors. Compass in $(A)$ and $(C)$ : $D=$ 959 dorsal, $\mathrm{L}=$ lateral, $\mathrm{P}=$ posterior. See also Table 3 and Figure 3-Source Data 1 and 2. 


\begin{tabular}{|c|c|c|c|c|c|c|c|c|c|c|}
\hline Gene & Approach & mCherry+ & mCherry- & Total & Efficiency & $\begin{array}{l}\text { Founders } \\
\text { Producing } \\
\text { Knock-in } \\
\text { (\#/Total) }\end{array}$ & $\begin{array}{l}\text { Knock- } \\
\text { ins } \\
\text { Sampled }\end{array}$ & $\begin{array}{c}\text { False } \\
\text { Positives }\end{array}$ & Confirmed & Correct \\
\hline Orco & $\begin{array}{c}\text { Direct } \\
\text { Injection }\end{array}$ & 180 & 365 & 545 & $33 \%$ & $43 \%(3 / 7)$ & 30 & 0 & 30 & $100 \%$ \\
\hline Ir8a & $\begin{array}{c}\text { Direct } \\
\text { Injection }\end{array}$ & 53 & 609 & 662 & $8 \%$ & $20 \%(4 / 20)$ & 5 & 0 & 5 & $100 \%$ \\
\hline$I r 76 b$ & $\begin{array}{c}\text { Direct } \\
\text { Injection }\end{array}$ & 79 & 184 & 263 & $30 \%$ & $100 \%(2 / 2)$ & 10 & 0 & 10 & $100 \%$ \\
\hline Ir25a & $\begin{array}{c}\text { Direct } \\
\text { Injection }\end{array}$ & 82 & 268 & 350 & $23 \%$ & $40 \%(2 / 5)$ & 6 & 0 & 6 & $100 \%$ \\
\hline Orco & Cross & 37 & 96 & 133 & $28 \%$ & $100 \%(3 / 3)$ & 2 & 1 & 1 & $50 \%$ \\
\hline Ir25a & Cross & 30 & 95 & 125 & $24 \%$ & $100 \%(2 / 2)$ & 30 & 5 & 25 & $83 \%$ \\
\hline
\end{tabular}

962 There are two ways to generate knock-ins via the HACK technique: by direct injection or by genetic cross (see Figure 2-Figure Supplement 1 and Methods for details) (Lin and Potter, 2016a). All four co-receptor genes were targeted using the direct injection approach; additionally, the crossing approach was tested with Orco and Ir25a. Knock-in efficiency, as measured by the number of flies having the mCherry+ marker divided by the total number of potentially HACKed flies, was high for all genes tested and both approaches. Efficiency appears to depend on the genetic locus, as has been previously demonstrated (Lin and Potter, 2016a). To further estimate the effort required to generate a HACK knock-in, we calculated the percentage of founder flies producing knock-in lines; this gives an indication of the number of independent crosses needed to successfully create a knock-in line. Two to five 
969 individual $\mathrm{G}_{0}$ starting crosses were sufficient to produce a knock-in. For each gene, a sample of individual knock-in lines were tested 970 via PCR genotyping, sequencing, and by crossing to a reporter line to confirm brain expression (knock-ins sampled). For all knock971 ins generated via the direct injection method, every fly tested represented a correctly targeted knock-in. However, for the cross 972 method, some lines had the mCherry+ marker and yet did not drive GFP expression in the brain when crossed to a reporter line 973 (labeled here as false positives). See also Table 1-Source Data 1 and Figure 2-Figure Supplement 1. 
974 Table 2

\begin{tabular}{|c|c|c|c|c|c|c|}
\hline Knock-in & Sample* & Antibody $^{\dagger}(A b)$ & $\mathrm{Ab}+$ cells & GFP+ cells & $\begin{array}{c}\text { Double-labeled } \\
\text { cells }\end{array}$ & Total cells \\
\hline Orco & Palp 1 (WM) & anti-Orco & 125 & 127 & 125 & 127 \\
\hline Orco & Palp 2 (WM) & anti-Orco & 112 & 111 & 108 & 115 \\
\hline \multirow[t]{4}{*}{ Orco } & Palp 6 (WM) & anti-Orco & 125 & 126 & 123 & 128 \\
\hline & & $\begin{array}{l}\text { Total across } \\
\text { samples: }\end{array}$ & 362 & 364 & 356 & 370 \\
\hline & & & $\begin{array}{l}\text { Proportion of } \\
\text { Ab+ cells that } \\
\text { are GFP+: }\end{array}$ & $\begin{array}{l}\text { Proportion of } \\
\text { GFP+ cells that } \\
\text { are Ab+: }\end{array}$ & $\begin{array}{l}\text { Proportion of all } \\
\text { cells that are } \\
\text { double labeled: }\end{array}$ & \\
\hline & & & 0.98 & 0.98 & 0.96 & \\
\hline Ir8a & Antenna 1 (Cryo) & anti-Ir8a & 20 & 21 & 20 & 21 \\
\hline Ir8a & Antenna 2 (Cryo) & anti-Ir8a & 24 & 24 & 24 & 24 \\
\hline Ir8a & Antenna 6 (Cryo) & anti-Ir8a & 40 & 43 & 40 & 43 \\
\hline Ir8a & Antenna 7 (Cryo) & anti-Ir8a & 12 & 13 & 12 & 13 \\
\hline Ir8a & Antenna 8 (Cryo) & anti-Ir8a & 16 & 16 & 16 & 16 \\
\hline Ir8a & Antenna 9 (Cryo) & anti-Ir8a & 42 & 42 & 41 & 43 \\
\hline \multirow[t]{4}{*}{ Ir8a } & Antenna 10 (Cryo) & anti-Ir8a & 41 & 40 & 40 & 41 \\
\hline & & $\begin{array}{l}\text { Total across } \\
\text { samples: }\end{array}$ & 195 & 199 & 193 & 201 \\
\hline & & & $\begin{array}{l}\text { Proportion of } \\
\text { Ab+ cells that } \\
\text { are GFP+: }\end{array}$ & $\begin{array}{l}\text { Proportion of } \\
\text { GFP+ cells that } \\
\text { are } A b+:\end{array}$ & $\begin{array}{l}\text { Proportion of all } \\
\text { cells that are } \\
\text { double labeled: }\end{array}$ & \\
\hline & & & 0.99 & 0.97 & 0.96 & \\
\hline Ir25a & Palp 1 (WM) & anti-Ir25a & 107 & 105 & 104 & 108 \\
\hline Ir25a & Palp 2 (WM) & anti-Ir25a & 86 & 85 & 85 & 86 \\
\hline Ir25a & Palp 3 (WM) & anti-Ir25a & 111 & 111 & 110 & 112 \\
\hline Ir25a & Palp 4 (WM) & anti-Ir25a & 94 & 94 & 94 & 94 \\
\hline \multirow[t]{4}{*}{ Ir25a } & Palp 5 (WM) & anti-Ir25a & 83 & 83 & 81 & 85 \\
\hline & & $\begin{array}{l}\text { Total across } \\
\text { samples: }\end{array}$ & 481 & 478 & 474 & 485 \\
\hline & & & $\begin{array}{l}\text { Proportion of } \\
\text { Ab+ cells that } \\
\text { are GFP+: }\end{array}$ & $\begin{array}{l}\text { Proportion of } \\
\text { GFP+ cells that } \\
\text { are } A b+:\end{array}$ & $\begin{array}{l}\text { Proportion of all } \\
\text { cells that are } \\
\text { double labeled: }\end{array}$ & \\
\hline & & & 0.99 & 0.99 & 0.98 & \\
\hline
\end{tabular}


975 Table 2. Validation of T2A-QF2 Knock-in Expression in the Antennae and Maxillary Palps.

$976 \quad$ Related to Figure 2.

977 To verify that the knock-in lines recapitulate the endogenous expression patterns of the target

978 genes, antennae or maxillary palps of flies containing the knock-ins driving GFP expression

979 were co-stained with the corresponding antibody $(\mathrm{Ab})$ (anti-Orco, anti-Ir8a, or anti-Ir25a). The

980 overlap of $\mathrm{Ab}+$ and GFP+ cells was examined, and a high correspondence between antibody

981 staining and knock-in driven GFP was found. ${ }^{*} \mathrm{WM}=$ whole-mount, Cryo $=$ cryosection. See also

982 Figure 2-Figure Supplement 3. 
Table 3

\begin{tabular}{|c|c|c|c|c|c|c|c|c|}
\hline Glomerulus $^{\dagger}$ & Sensillum & $\begin{array}{c}\text { Tuning } \\
\text { Receptor(s) }\end{array}$ & $\begin{array}{c}\text { Original } \\
\text { Co-receptor(s) }\end{array}$ & Orco-T2A-QF2 & Ir8a-T2A-QF2 & Ir76b-T2A-QF2 & Ir25a-T2A-QF2 & References \\
\hline $\mathrm{D}$ & Ab9A & $\begin{array}{l}\text { Or69aA, } \\
\text { Or69aB }\end{array}$ & Orco & Yes & Variable & No & No & $\begin{array}{l}\text { (Couto et al., 2005; } \\
\text { Fishilevich and Vosshall, } \\
\text { 2005) }\end{array}$ \\
\hline DA1 & At1A & Or67d & Orco & Yes & No & Variable & Yes & $\begin{array}{c}\text { (Couto et al., 2005; } \\
\text { Fishilevich and Vosshall, } \\
\text { 2005; Kurtovic et al., 2007) }\end{array}$ \\
\hline DA2 & Ab4B & $\begin{array}{l}\text { Or56a, } \\
\text { Or33a }\end{array}$ & Orco & Yes & No & No & Yes & $\begin{array}{c}\text { (Couto et al., 2005; } \\
\text { Fishilevich and Vosshall, } \\
\text { 2005) }\end{array}$ \\
\hline DA3 & Ai2B (At2B) & Or23a & Orco & Yes & No & No & Yes & $\begin{array}{c}\text { (Couto et al., 2005; } \\
\text { Fishilevich and Vosshall, } \\
\text { 2005; Lin and Potter, 2015) }\end{array}$ \\
\hline DA4I & Ai3C (At3C) & Or43a & Orco & Yes & No & No & Yes & $\begin{array}{c}\text { (Couto et al., 2005; } \\
\text { Fishilevich and Vosshall, } \\
\text { 2005; Lin and Potter, 2015) }\end{array}$ \\
\hline $\mathrm{DA} 4 \mathrm{~m}$ & Ai3B (At3B) & Or2a & Orco & Yes & No & No & Yes & $\begin{array}{l}\text { (Couto et al., 2005; Lin and } \\
\text { Potter, 2015) }\end{array}$ \\
\hline DC1 & Ai3A (At3A) & $\begin{array}{l}\text { Or19a, } \\
\text { Or19b }\end{array}$ & Orco & Yes & No & No & Yes & $\begin{array}{c}\text { (Couto et al., 2005; } \\
\text { Fishilevich and Vosshall, } \\
\text { 2005; Lin and Potter, 2015) }\end{array}$ \\
\hline DC2 & $\mathrm{Ai1A}(\mathrm{Ab6A})$ & Or13a & Orco & Yes & No & No & Yes & $\begin{array}{l}\text { (Couto et al., 2005; } \\
\text { Fishilevich and Vosshall, } \\
\text { 2005; Lin and Potter, 2015) }\end{array}$ \\
\hline DC3 & $\mathrm{Ai} 2 \mathrm{~A}(\mathrm{At} 2 \mathrm{~A})$ & Or83c & Orco & Yes & No & Yes & Variable & $\begin{array}{c}\text { (Couto et al., 2005; } \\
\text { Fishilevich and Vosshall, } \\
\text { 2005; Lin and Potter, 2015) }\end{array}$ \\
\hline DL1 & Ab1D & $\begin{array}{l}\text { Or10a, } \\
\text { Gr10a }\end{array}$ & Orco & Yes & Yes & Yes & Yes & $\begin{array}{c}\text { (Couto et al., 2005; } \\
\text { Fishilevich and Vosshall, } \\
\text { 2005) }\end{array}$ \\
\hline DL3 & At4B & $\begin{array}{l}\text { Or65a, } \\
\text { Or65b, } \\
\text { Or65c }\end{array}$ & Orco & Yes & No & No & Yes & $\begin{array}{l}\text { (Couto et al., 2005; } \\
\text { Fishilevich and Vosshall, } \\
\text { 2005) }\end{array}$ \\
\hline DL4 & Ab10B & Or49a, Or85f & Orco & Yes & No & No & Yes & $\begin{array}{l}\text { (Couto et al., 2005; } \\
\text { Fishilevich and Vosshall, } \\
\text { 2005) }\end{array}$ \\
\hline DL5 & $\mathrm{Ab} 4 \mathrm{~A}$ & Or7a & Orco & Yes & No & No & Variable & (Couto et al., 2005) \\
\hline
\end{tabular}




\begin{tabular}{|c|c|c|c|c|c|c|c|c|}
\hline DM1 & Ab1A & Or42b & Orco & Yes & No & No & Yes & $\begin{array}{c}\text { (Couto et al., 2005; } \\
\text { Fishilevich and Vosshall, } \\
\text { 2005) }\end{array}$ \\
\hline DM2 & Ab3A & $\begin{array}{l}\text { Or22a, } \\
\text { Or22b }\end{array}$ & Orco & Yes & No & No & Yes & $\begin{array}{l}\text { (Couto et al., 2005; } \\
\text { Fishilevich and Vosshall, } \\
\text { 2005) }\end{array}$ \\
\hline DM3 & Ab5B & $\begin{array}{l}\text { Or47a, } \\
\text { Or33b }\end{array}$ & Orco & Yes & No & No & No & $\begin{array}{c}\text { (Couto et al., 2005; } \\
\text { Fishilevich and Vosshall, } \\
\text { 2005) }\end{array}$ \\
\hline DM4 & Ab2A & Or59b & Orco & Yes & No & No & Yes & (Couto et al., 2005) \\
\hline DM5 & $A b 2 B$ & $\begin{array}{l}\text { Or85a, } \\
\text { Or33b }\end{array}$ & Orco & Yes & No & No & No & $\begin{array}{c}\text { (Couto et al., 2005; } \\
\text { Fishilevich and Vosshall, } \\
\text { 2005) }\end{array}$ \\
\hline DM6 & Ab10A & Or67a & Orco & Yes & No & No & Yes & (Couto et al., 2005) \\
\hline VA1d & At4C & Or88a & Orco & Yes & No & No & Yes & $\begin{array}{c}\text { (Couto et al., 2005; } \\
\text { Fishilevich and Vosshall, } \\
\text { 2005) }\end{array}$ \\
\hline VA1v & At4A & Or47b & Orco & Yes & No & No & Yes & $\begin{array}{c}\text { (Couto et al., 2005; } \\
\text { Fishilevich and Vosshall, } \\
\text { 2005) }\end{array}$ \\
\hline VA2 & Ab1B & Or92a & Orco & Yes & No & No & Yes & $\begin{array}{c}\text { (Couto et al., 2005; } \\
\text { Fishilevich and Vosshall, } \\
\text { 2005) }\end{array}$ \\
\hline VA3 & Ab9B & Or67b & Orco & Yes & Yes & Yes & Yes & $\begin{array}{c}\text { (Couto et al., 2005; } \\
\text { Fishilevich and Vosshall, } \\
\text { 2005) }\end{array}$ \\
\hline VA4 & Pb3B & Or85d & Orco & Yes & No & No & Yes & (Couto et al., 2005) \\
\hline VA5 & Ai1B (Ab6B) & Or49b & Orco & Yes & Yes & Yes & Yes & $\begin{array}{l}\text { (Couto et al., 2005; Lin anc } \\
\text { Potter, 2015) }\end{array}$ \\
\hline VA6 & Ab5A & Or82a & Orco & Yes & Yes & Yes & Yes & $\begin{array}{c}\text { (Couto et al., 2005; } \\
\text { Fishilevich and Vosshall, } \\
\text { 2005) }\end{array}$ \\
\hline VA7I & $\mathrm{Pb} 2 \mathrm{~B}$ & Or46a & Orco & Yes & No & No & Yes & $\begin{array}{l}\text { (Couto et al., 2005; } \\
\text { Fishilevich and Vosshall, } \\
\text { 2005) }\end{array}$ \\
\hline VA7m & UNK & UNK & Orco & Yes & No & Variable & Yes & (Couto et al., 2005) \\
\hline
\end{tabular}




\begin{tabular}{|c|c|c|c|c|c|c|c|c|}
\hline VC1 & $\mathrm{Pb} 2 \mathrm{~A}$ & $\begin{array}{l}\text { Or33c, } \\
\text { Or85e }\end{array}$ & Orco & Yes & No & No & Yes & $\begin{array}{l}\text { (Couto et al., 2005; } \\
\text { Fishilevich and Vosshall, } \\
\text { 2005) }\end{array}$ \\
\hline VC2 & $\mathrm{Pb} 1 \mathrm{~B}$ & Or71a & Orco & Yes & No & No & Yes & $\begin{array}{l}\text { (Couto et al., 2005; } \\
\text { Fishilevich and Vosshall, } \\
\text { 2005) }\end{array}$ \\
\hline VC4 & Ab7B & Or67c & Orco & Yes & No & Yes & Yes & (Couto et al., 2005) \\
\hline VM2 & Ab8A & Or43b & Orco & Yes & No & No & No & (Couto et al., 2005) \\
\hline VM3 & Ab8B & Or9a & Orco & Yes & No & No & No & (Couto et al., 2005) \\
\hline VM5d & Ab3B & $\begin{array}{l}\text { Or85b?, } \\
\text { Or98b? }\end{array}$ & Orco & Yes & Variable & No & Yes & (Couto et al., 2005) \\
\hline VM5v & Ab7A & Or98a & Orco & Yes & Yes & No & Yes & $\begin{array}{c}\text { (Couto et al., 2005; } \\
\text { Fishilevich and Vosshall, } \\
\text { 2005) }\end{array}$ \\
\hline VM7d & $\mathrm{Pb} 1 \mathrm{~A}$ & Or42a & Orco & Yes & No & No & Yes & $\begin{array}{l}\text { (Couto et al., 2005; Endo et } \\
\text { al., 2007; Fishilevich and } \\
\text { Vosshall, 2005) }\end{array}$ \\
\hline VM7v (1) & $\mathrm{Pb} 3 \mathrm{~A}$ & Or59c & Orco & Yes & No & No & Yes & $\begin{array}{c}\text { (Couto et al., 2005; Endo et } \\
\text { al., 2007) }\end{array}$ \\
\hline VC3 & Ac3B & Or35a & Orco, Ir76b & Yes & Yes & Yes & Yes & $\begin{array}{l}\text { (Couto et al., 2005; } \\
\text { Fishilevich and Vosshall, } \\
\text { 2005; Silbering et al., 2011) }\end{array}$ \\
\hline V & Ab1C & $\begin{array}{l}\text { Gr21a, } \\
\text { Gr63a }\end{array}$ & $\mathrm{N} / \mathrm{A}$ & Yes & No & No & Yes & $\begin{array}{l}\text { (Couto et al., 2005; Jones et } \\
\text { al., 2007; Kwon et al., 2007) }\end{array}$ \\
\hline DC4 & $\begin{array}{l}\text { Sacculus, } \\
\text { Chamber III }\end{array}$ & Ir64a & Ir8a & Variable & Yes & No & Yes & $\begin{array}{l}\text { (Ai et al., 2013; Ai et al., } \\
\text { 2010; Silbering et al., 2011) }\end{array}$ \\
\hline DL2d & Ac3A & $\operatorname{Ir} 75 b$ & Ir8a & Yes & Yes & No & Yes & $\begin{array}{l}\text { (Prieto-Godina et al., 2017; } \\
\quad \text { Silbering et al., 2011) }\end{array}$ \\
\hline DL2v & Ac3A & $\operatorname{Ir} 75 \mathrm{c}$ & Ir8a & Yes & Yes & No & Yes & $\begin{array}{l}\text { (Prieto-Godina et al., 2017; } \\
\quad \text { Silbering et al., 2011) }\end{array}$ \\
\hline DP1I & Ac2 & Ir75a & Ir8a & Yes & Yes & Yes & Yes & (Silbering et al., 2011) \\
\hline DP1m & $\begin{array}{l}\text { Sacculus, } \\
\text { Chamber III }\end{array}$ & Ir64a & Ir8a & No & Yes & Yes & Yes & $\begin{array}{l}\text { (Ai et al., 2013; Ai et al., } \\
\text { 2010; Silbering et al., 2011) }\end{array}$ \\
\hline VL2a & Ac4 & Ir84a & Ir8a & Yes & Yes & Yes & Yes & (Silbering et al., 2011) \\
\hline
\end{tabular}




\begin{tabular}{|c|c|c|c|c|c|c|c|c|}
\hline VL2p & Ac1 & Ir31a & Ir8a & No & Yes & Yes & Yes & (Silbering et al., 2011) \\
\hline VC5 & Ac2 & Ir41a & $\begin{array}{l}\text { Ir8a, Ir25a, } \\
\quad \text { Ir76b }\end{array}$ & No & Yes & Yes & Yes & $\begin{array}{l}\text { (Hussain et al., 2016; Min et } \\
\text { al., 2013; Silbering et al., } \\
\text { 2011) }\end{array}$ \\
\hline VM1 & Ac1 & Ir92a & $\begin{array}{l}\text { Ir8a, Ir25a, } \\
\text { Ir76b }\end{array}$ & No & Yes & Yes & Yes & $\begin{array}{c}\text { (Min et al., 2013; Silbering } \\
\text { et al., 2011) }\end{array}$ \\
\hline VM4 & Ac4 & Ir76a & $\begin{array}{l}\text { Ir8a, Ir25a, } \\
\quad \text { Ir76b }\end{array}$ & Yes & Yes & Yes & Yes & $\begin{array}{l}\text { (Benton et al., 2009; Min et } \\
\text { al., 2013; Silbering et al., } \\
\text { 2011) }\end{array}$ \\
\hline $\mathrm{VL} 1$ & $\begin{array}{c}\text { Ac1, Ac2 } \\
\text { Ac4 }\end{array}$ & $\operatorname{lr} 75 d$ & Ir25a & Yes & Yes & Yes & Yes & (Silbering et al., 2011) \\
\hline $\begin{array}{l}\text { VM6v } \\
\text { (VM6) }\end{array}$ & Ac1 & Rh50, Amt & Ir25a & No & Yes (weak) & No & Yes & $\begin{array}{c}\text { (Chai et al., 2019; Li et al., } \\
\text { 2016; Schlegel et al., 2021; } \\
\text { Vulpe et al., 2021), } \\
\text { This paper }\end{array}$ \\
\hline $\begin{array}{l}\text { VM6m } \\
\text { (new) }\end{array}$ & $\begin{array}{l}\text { Sacculus, } \\
\text { Chamber III }\end{array}$ & Rh50, Amt & $\begin{array}{l}\text { N/A (this } \\
\text { paper) }\end{array}$ & No & Yes (weak) & No & Yes & $\begin{array}{c}\text { (Chai et al., 2019; Li et al., } \\
\text { 2016; Schlegel et al., 2021; } \\
\text { Vulpe et al., 2021), } \\
\text { This paper }\end{array}$ \\
\hline $\begin{array}{l}\mathrm{VM}^{*} \\
\text { (new) }\end{array}$ & $\begin{array}{l}\text { Sacculus, } \\
\text { Chamber III }\end{array}$ & Rh50, Amt & $\begin{array}{l}\text { N/A (this } \\
\text { paper) }\end{array}$ & No & Yes (strong) & No & Yes & $\begin{array}{c}\text { (Chai et al., 2019; Li et al., } \\
\text { 2016; Schlegel et al., 2021; } \\
\text { Vulpe et al., 2021), } \\
\text { This paper }\end{array}$ \\
\hline VP1d & $\begin{array}{l}\text { Sacculus, } \\
\text { Chamber II }\end{array}$ & Ir40a, Ir93a & Ir25a & No & No & No & Yes & $\begin{array}{l}\text { (Enjin et al., 2016; Frank et } \\
\text { al., 2017; Knecht et al., } \\
\text { 2017; Knecht et al., 2016; } \\
\text { Marin et al., 2020; Silbering } \\
\text { et al., 2011) }\end{array}$ \\
\hline VP1I & $\begin{array}{l}\text { Sacculus, } \\
\text { Chamber I }\end{array}$ & Ir21a, Ir93a & Ir25a & No & No & No & Yes & $\begin{array}{l}\text { (Frank et al., 2017; Knecht } \\
\text { et al., 2017; Knecht et al., } \\
\text { 2016; Marin et al., 2020; } \\
\text { Silbering et al., 2011) }\end{array}$ \\
\hline VP1m & $\begin{array}{l}\text { Sacculus, } \\
\text { Chamber I }\end{array}$ & Ir68a, Ir93a & Ir25a & No & No & No & Yes & $\begin{array}{l}\text { (Frank et al., 2017; Knecht } \\
\text { et al., 2017; Knecht et al., } \\
\text { 2016; Marin et al., 2020; } \\
\text { Silbering et al., 2011) }\end{array}$ \\
\hline VP2 & Arista & $\begin{array}{l}\text { Gr28b.d, } \\
\text { Ir93a }\end{array}$ & Ir25a & No & No & No & Yes & $\begin{array}{c}\text { (Enjin et al., 2016; Frank et } \\
\text { al., 2017; Marin et al., 2020; } \\
\text { Miwa et al., 2018; Ni et al., } \\
\text { 2013) }\end{array}$ \\
\hline
\end{tabular}




\begin{tabular}{|c|c|c|c|c|c|c|c|c|}
\hline VP3 & Arista & Ir21a, Ir93a & Ir25a & No & No & No & Yes & $\begin{array}{l}\text { (Budelli et al., 2019; Enjin et } \\
\text { al., 2016; Frank et al., 2017; } \\
\text { Silbering et al., 2011) }\end{array}$ \\
\hline VP4 & $\begin{array}{l}\text { Sacculus, } \\
\text { Chambers I } \\
+ \text { II }\end{array}$ & Ir40a, Ir93a & Ir25a & No & No & No & Yes & $\begin{array}{l}\text { (Enjin et al., 2016; Frank et } \\
\text { al., 2017; Knecht et al., } \\
\text { 2017; Knecht et al., 2016; } \\
\text { Marin et al., 2020; Silbering } \\
\text { et al., 2011) }\end{array}$ \\
\hline VP5 & $\begin{array}{l}\text { Sacculus, } \\
\text { Chamber II }\end{array}$ & Ir68a, Ir93a & Ir25a & No & No & No & Yes & $\begin{array}{c}\text { (Frank et al., 2017; Knecht } \\
\text { et al., 2017; Marin et al., } \\
\text { 2020) }\end{array}$ \\
\hline
\end{tabular}

984 Table 3. Summary of Expression Patterns for All Knock-in Lines. Related to Figures 3 - 5.

985 Summarized here are all of the OSN classes innervating the 58 antennal lobe glomeruli ${ }^{\dagger}$; their corresponding sensilla and tuning

986 receptors; the previously reported (original) co-receptors they express; and whether or not each of the co-receptor knock-in lines

987 labels those glomeruli. Variable indicates that the glomerulus was labeled in $<50 \%$ of brains examined in the given knock-in line.

988 Sensilla or glomeruli that have been renamed or reclassified have their former nomenclature listed in parentheses. Question marks

989 indicate expression that has been reported but not functionally validated. ${ }^{\dagger}$ The VM6 subdivisions (VM6v, VM6m, VM6I) are separated

990 in this table for clarity but counted together as one glomerulus in accordance with Schlegel et al., 2021. *VM6I was initially named

991 VC6 in version 1 of our pre-print (Task et al., 2020) but was reclassified using additional data from EM reconstructions in the AL and

992 immunohistochemical experiments in the periphery (see Figure 5). See also Figure 3-Source Data 1 and 2. 
993 Table 4

\begin{tabular}{|c|c|c|c|c|c|}
\hline Genotype & Sample & Ir8a+ cells & GFP+ cells & Double-labeled cells & Total cells \\
\hline Rh50-Gal4 > GFP & 20210226 a1 & 18 & 9 & 2 & 25 \\
\hline Rh50-Gal4 > GFP & 20210226 a2 & 22 & 15 & 4 & 33 \\
\hline Rh50-Gal4 > GFP & 20210226 a3 & 41 & 22 & 7 & 56 \\
\hline Rh50-Gal4 > GFP & 20210226 a4 & 41 & 14 & 5 & 50 \\
\hline Rh50-Gal4 > GFP & 20210129 a1 & 26 & 20 & 9 & 37 \\
\hline Rh50-Gal4 > GFP & 20210129 a2 & 32 & 24 & 7 & 49 \\
\hline Rh50-Gal4 > GFP & 20210129 a3 & 29 & 19 & 7 & 41 \\
\hline Rh50-Gal4 > GFP & 20210216 a1 & 26 & 21 & 8 & 39 \\
\hline Rh50-Gal4 > GFP & 20210216 a2 & 30 & 18 & 7 & 41 \\
\hline Rh50-Gal4 > GFP & 20210216 a3 & 34 & 23 & 8 & 49 \\
\hline \multirow[t]{4}{*}{ Rh50-Gal4 > GFP } & 20210216 a4 & 34 & 23 & 9 & 48 \\
\hline & $\begin{array}{l}\text { Total across } \\
\text { samples: }\end{array}$ & 333 & 208 & 73 & 468 \\
\hline & & $\begin{array}{l}\text { Proportion of Ir8a+ } \\
\text { cells that are GFP+: }\end{array}$ & $\begin{array}{l}\text { Proportion of GFP+ } \\
\text { cells that are Ir8a+: }\end{array}$ & $\begin{array}{l}\text { Proportion of all cells } \\
\text { that are double labeled: }\end{array}$ & \\
\hline & & 0.22 & 0.35 & 0.16 & \\
\hline
\end{tabular}

994 Table 4. Co-expression of Rh50 and Ir8a in the Sacculus. Related to Figure 5.

995 Antennal cryosections of $R h 50-G a l 4>$ GFP flies were stained with an anti-Ir8a antibody and the

996 overlap of Ir8a+ and GFP+ cells was quantified in the sacculus. $22 \%$ of Ir8a+ cells expressed

997 Rh50, $35 \%$ of Rh50+ cells expressed Ir8a, and $16 \%$ of all cells were double labeled. $\mathrm{N}=11$. 


\section{Figure Supplements}

\section{$999 \quad$ Figure 2-Figure Supplement 1}

A

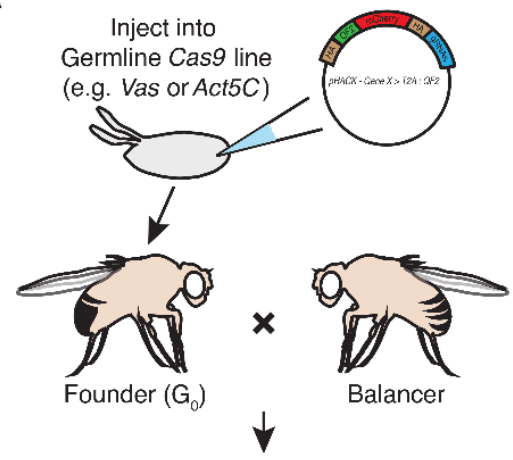

Progeny $\left(G_{1}\right)$

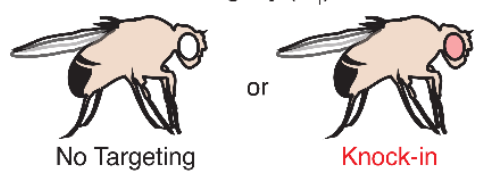

B

On Autosome (e.g. Orco, Ir76b, Ir25a):

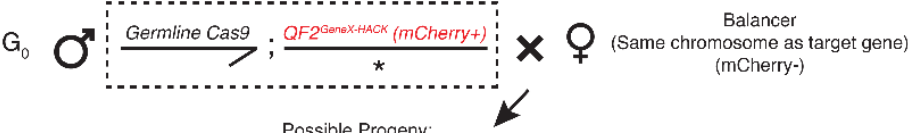

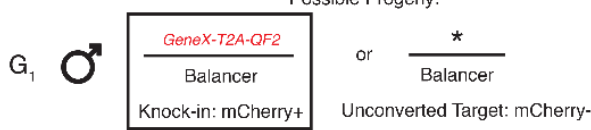

On X (e.g. Ir8a):

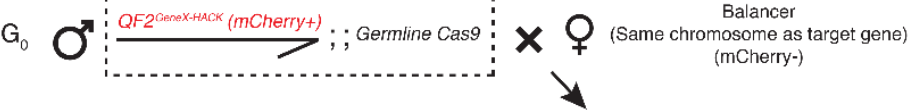

$\mathrm{G}_{1}$

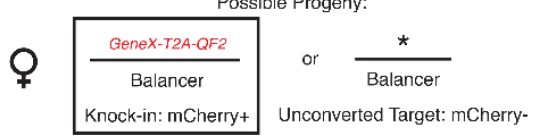

C

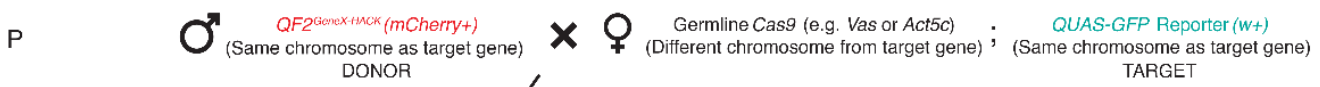

O

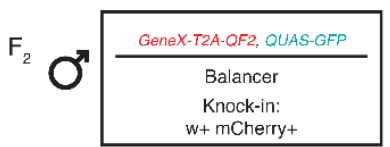

$\frac{\text { QF2 Genex-HACK }}{\text { Balancer }}$
Donor:
W- mCherry+

E

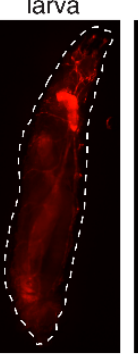

pupa

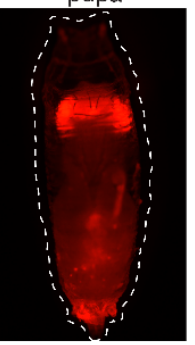

F

F

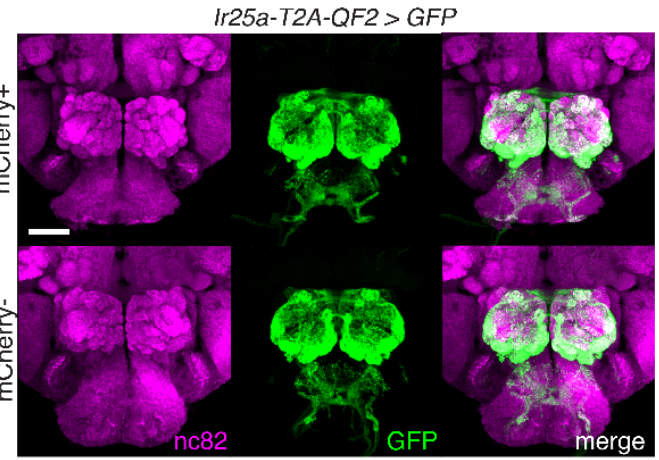

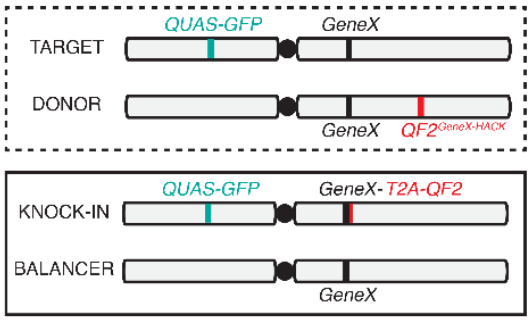

Ir25a-T2A-QF2 (mCherry+)

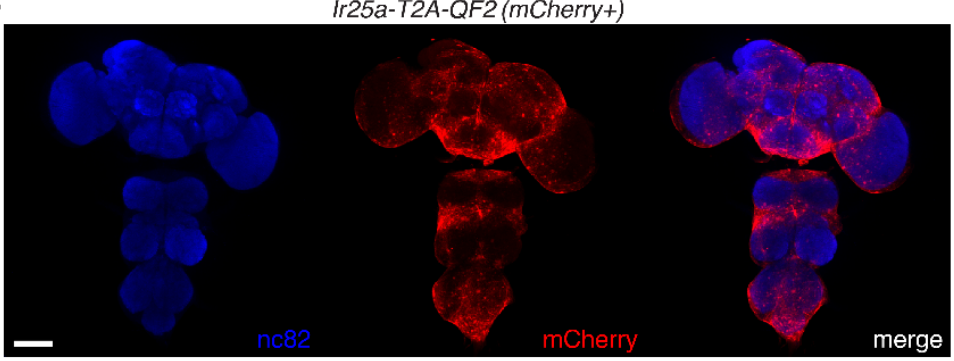

G

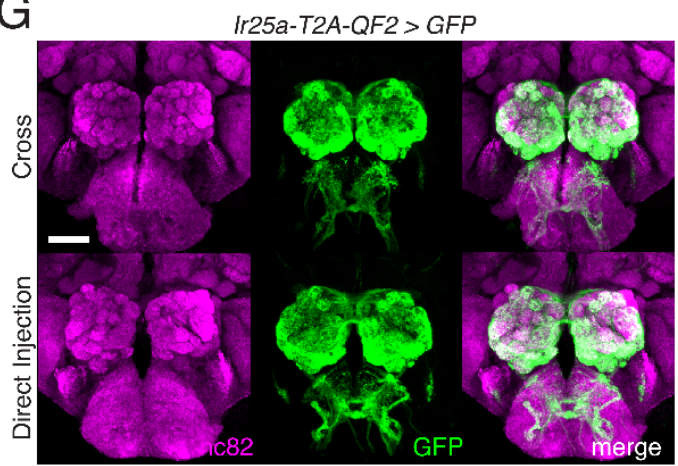


1001 Approach Comparison.

1002 A. Schematic of the direct injection method to generate a HACK knock-in. The HACK KI 1003 construct is injected into a germline Cas9 expressing embryo (both Act5C-Cas9 and Vas-Cas9 1004 yielded successful knock-ins; see Methods for more details). This approach requires one 1005 genetic cross to generate a knock-in: injected founder flies $\left(\mathrm{G}_{0}\right)$ are individually crossed to 1006 balancer flies, and progeny $\left(G_{1}\right)$ are screened for the mCherry knock-in marker. Each mCherry+ $1007 \mathrm{G}_{1}$ represents an independent knock-in event; thus, single mCherry+ flies are used to establish 1008 knock-in lines. B. Details of the genetic crosses used to establish knock-in lines for each target gene using the direct injection approach. For genes on autosomes (Orco and Ir76b, $3^{\text {rd }}$ chromosome; Ir25a, $2^{\text {nd }}$ chromosome), a Cas9 line on the $\mathrm{X}$ chromosome was used, and mCherry+ male progeny selected to remove the Cas9 after successful HACKing. For Ir8a on the $\mathrm{X}$ chromosome, a Cas9 line on the $3^{\text {rd }}$ chromosome was used which was marked with GFP to facilitate its removal in subsequent generations. In addition to mCherry marker screening, PCR genotyping, and sequencing, knock-in lines were confirmed by crossing to a QUAS reporter line and checking brain expression, either at the $\mathrm{G}_{2}$ stage, or by crossing $\mathrm{G}_{0}$ flies to balanced reporter lines to immediately check $\mathrm{G}_{1}$ brain expression. $\mathbf{C}$. Details of using the crossing method to generate HACK knock-ins. This involves the creation of donor lines, in which the HACK

1018 targeting construct is inserted into the genome, such as through $P$-element insertion or $\Phi$ C31 1019 integration. Donor lines are crossed to a germline Cas9 line (Parental). The progeny, $\mathrm{F}_{1}$, have 1020 all the components necessary for a knock-in to occur (gRNAs, Cas9, HDR cassette). An 1021 additional cross is required to select germline HACKing events at the $F_{2}$ generation. Because 1022 the donor lines also express mCherry, as do the knock-ins, the target chromosome must be 1023 marked with a different marker (such as w+) for knock-in selection (see chromosome 1024 schematics at the right, illustrating the locations of the genetic elements in the $F_{1}$ and $F_{2}$ 1025 generations). $F_{2}$ knock-ins will have both markers (in this case, mCherry+ w+), while donor flies 
1026 will only have one (mCherry+ w-). The cross approach was tested with two of the four genetic 1027 targets (Orco and Ir25a) and successfully produced knock-in lines for both. However, the cross 1028 approach can produce false positives (see Table 1 and Table 1-Source Data 1) and is more 1029 time consuming. The direct injection approach is recommended for generation of targeted 1030 knock-ins. D. Knock-in screening can be performed at the larval (left) and pupal (right) stages.

1031 E. The 3XP3-mCherry marker is expressed throughout the adult fly brain and ventral nerve 1032 cord. Nc82 channel has been pseudo-colored blue for clarity. F. The marker cassette is flanked 1033 by loxP sites and can be removed through Cre recombination (see Methods for details) (Siegal 1034 and Hartl, 1996). The 3XP3-mCherry marker does not affect knock-in expression. Ir25a-T2A1035 QF2-driven GFP expression in the brain was similar before (top row) and after (bottom row) 1036 Cre-mediated removal of the mCherry marker. G. No difference was found in the expression 1037 patterns of Ir25a-T2A-QF2 knock-in lines generated by the two HACK methods, genetic cross 1038 (top) and direct injection (bottom). For (F) and (G): $N=5$ for direct injection; $N=4$ for cross, 1039 mCherry+; $\mathrm{N}=3$ for cross, mCherry-. Expression patterns were comparable across all three 1040 groups. See also Table 1 and Table 1-Source Data 1. Scale bars: $100 \mu \mathrm{m}$ in (E), $50 \mu \mathrm{m}$ in (F$1041 \mathrm{G})$. 
A

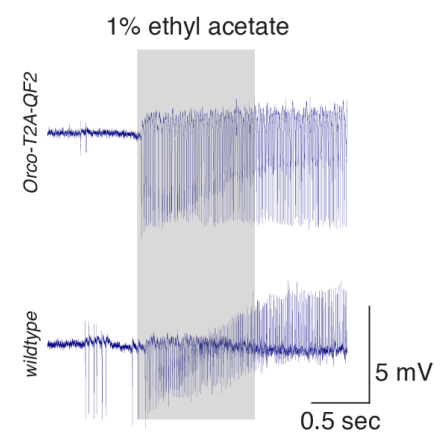

C

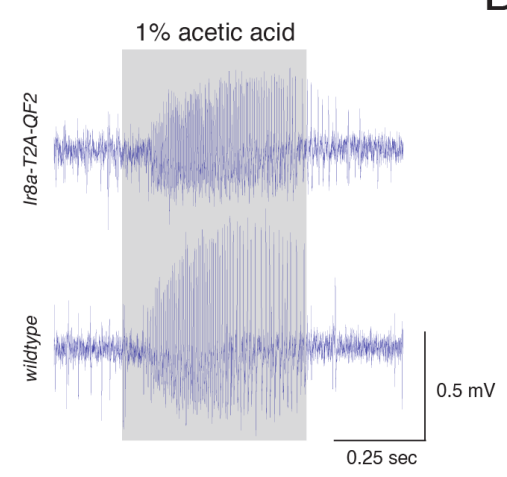

$E$

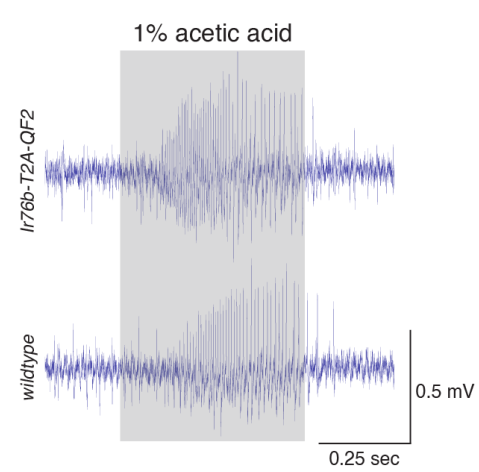

$\mathrm{G}$

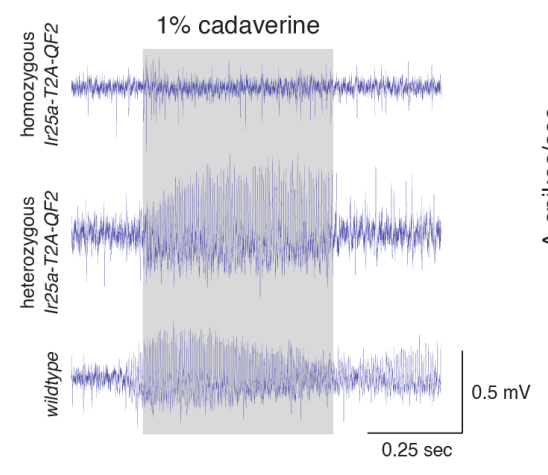

$\mathrm{F}$

$\mathrm{H}$
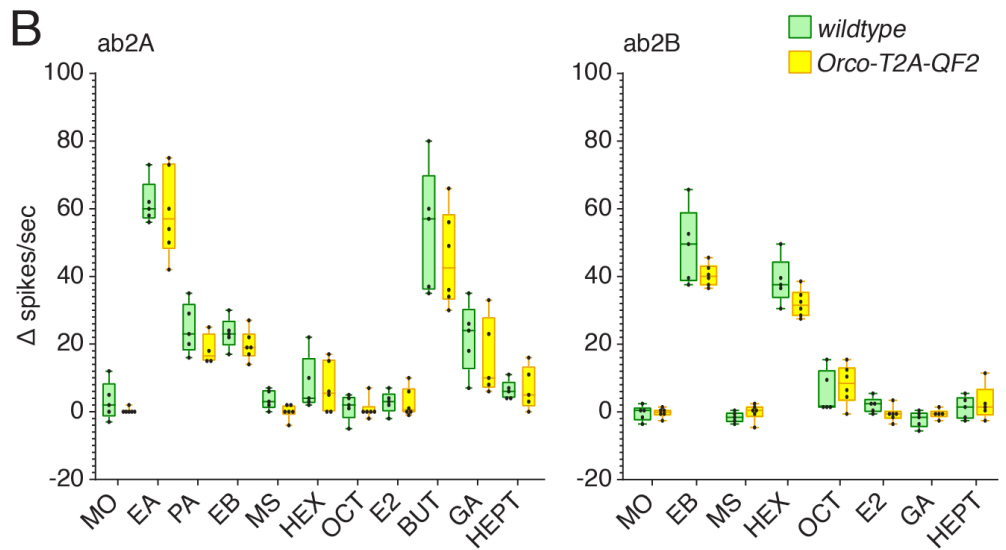

D
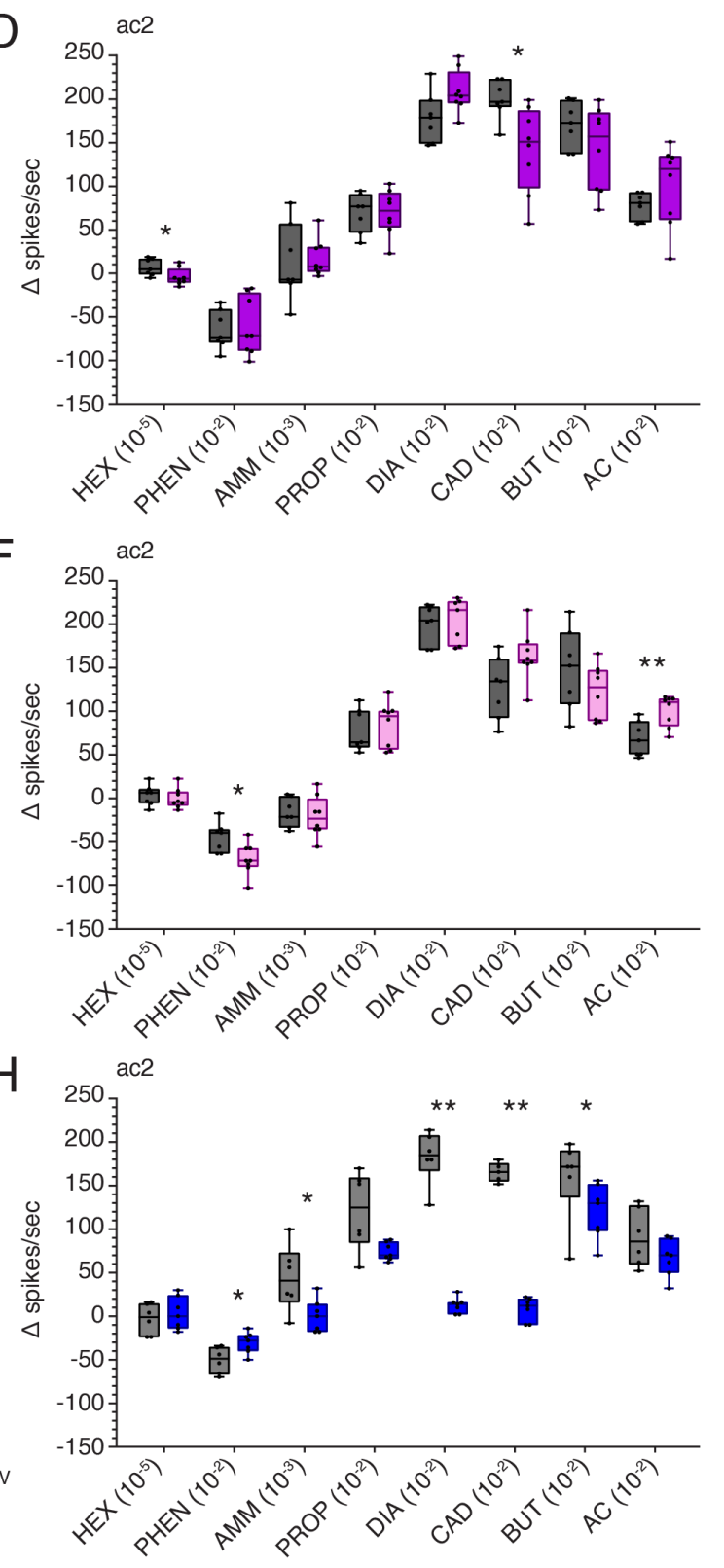

$\square$ wildtype

$\square$ Ir76b-T2A-QF2 
A. Orco knock-in SSR experiments were performed in ab2 sensilla. Responses to ten odorants

1045 plus mineral oil control were compared in wildtype and homozygous Orco-T2A-QF2 sensilla.

1046 Shown are example responses to 1\% ethyl acetate. B. ab2 sensilla contain two neurons: A (left)

1047 and B (right). No differences were found in the responses of these neurons between the two

1048 genotypes across all odorants tested (Mann-Whitney $U$ test, $p>0.05$ ). A small difference in

1049 baseline (pre-stimulus) activity was observed, which can be seen in (A). Fewer stimuli are

1050 shown for ab2B because odorants strongly activating the A neuron obscured the B neuron

1051 responses. $\mathrm{N}=5$ for wildtype, $\mathrm{N}=6$ for Orco-T2A-QF2. Abbreviations: $\mathrm{MO}$ mineral oil, EA ethyl

1052 acetate, PA pentyl acetate, EB ethyl butyrate, MS methyl salicylate, HEX hexanol, OCT 1-octen-

1053 3-ol, E2 e2-hexenal, BUT 2,3-butanedione, GA geranyl acetate, HEPT 2-heptanone. C.-H. IrCo

1054 knock-in SSR experiments were performed in ac2 sensilla. Responses to eight odorants were

1055

compared in wildtype and homozygous knock-in flies. C. Example responses to $1 \%$ acetic acid

1056

in Ir8a-T2A-QF2 and wildtype sensilla. D. There was a small but significant difference in

1057

responses between the two genotypes to hexanol (Mann-Whitney $U$ test, $p=0.0286$ ) and

1058

cadaverine (Mann-Whitney $U$ test, $p=0.0106$ ), which are not typically considered to be Ir8a-

1059

dependent odorants (Silbering et al., 2011). Responses to all other stimuli were not significantly

1060

different between genotypes. $\mathrm{N}=7$ for wildtype, $\mathrm{N}=8$ for Ir8a-T2A-QF2. E. Example responses

1061 to $1 \%$ acetic acid in Ir76b-T2A-QF2 and wildtype flies. F. There were small but significant

1062 differences in responses to phenethylamine (Mann-Whitney $U$ test, $p=0.012$ ) and acetic acid

1063 (Mann-Whitney $U$ test, $p=0.0087$ ) between Ir76b-T2A-QF2 and wildtype flies. Responses to

1064 the other stimuli were not significantly different between genotypes. $\mathrm{N}=7$ for wildtype, $\mathrm{N}=8$ for

1065 Ir76b-T2A-QF2. G. Homozygous Ir25a-T2A-QF2 flies lost responses to most amines,

1066 recapitulating an Ir25a mutant phenotype (Abuin et al., 2011; Silbering et al., 2011).

1067 Heterozygous Ir25a-T2A-QF2 flies with one wildtype copy of Ir25a had normal responses.

1068 Shown are example responses to $1 \%$ cadaverine in homozygous Ir25a-T2A-QF2, heterozygous 
1069 Ir25a-T2A-QF2, and wildtype ac2 sensilla. H. Homozygous Ir25a-T2A-QF2 flies had strongly 1070 reduced or abolished responses to phenethylamine (Mann-Whitney $U$ test, $p=0.039$ ), ammonia 1071 (Mann-Whitney $U$ test, $p=0.0338$ ), 1,4-diaminobutane (Mann-Whitney $U$ test, $p=0.0012$ ), 1072 cadaverine (Mann-Whitney $U$ test, $p=0.0025$ ), and 2,3-butanedione (Mann-Whitney $U$ test, $p=$ 1073 0.0472). Responses to the other stimuli were not significantly different between genotypes. $\mathrm{N}=$ 10746 for wildtype, $\mathrm{N}=7$ for homozygous Ir25a-T2A-QF2. Abbreviations in (D-H): PO paraffin oil, 1075 HEX hexanol, PHEN phenethylamine, AMM ammonia, PROP propanal, DIA 1,4-diaminobutane, 1076 CAD cadaverine, BUT 2,3-butanedione, AC acetic acid. See Figure 2-Source Data 1 for all $U$ 1077 and $p$ values. 


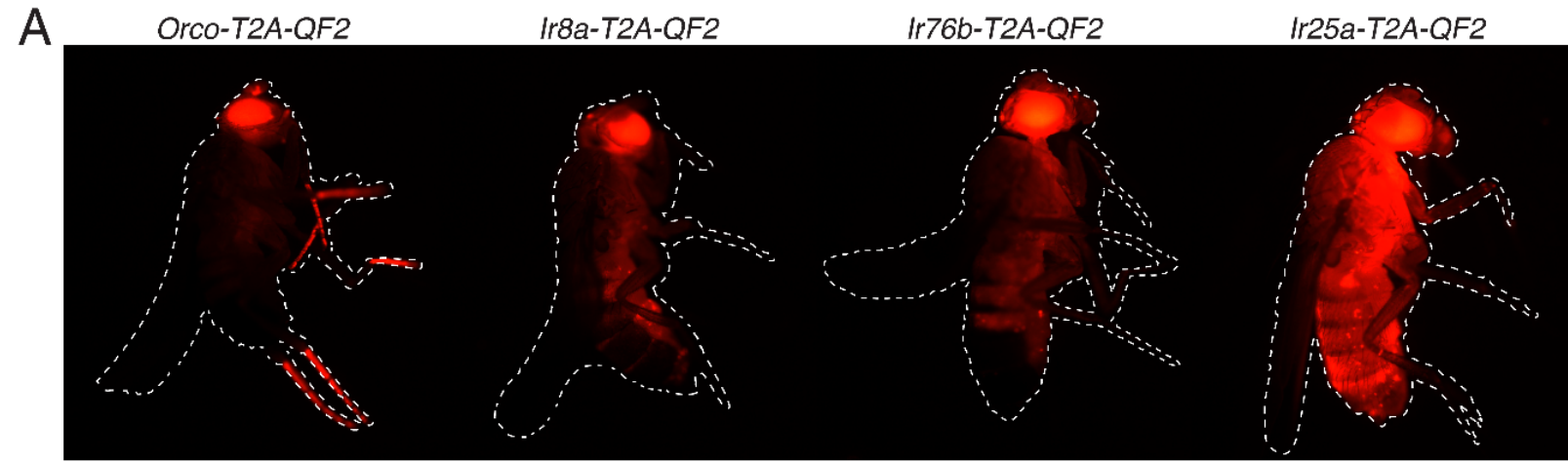

B

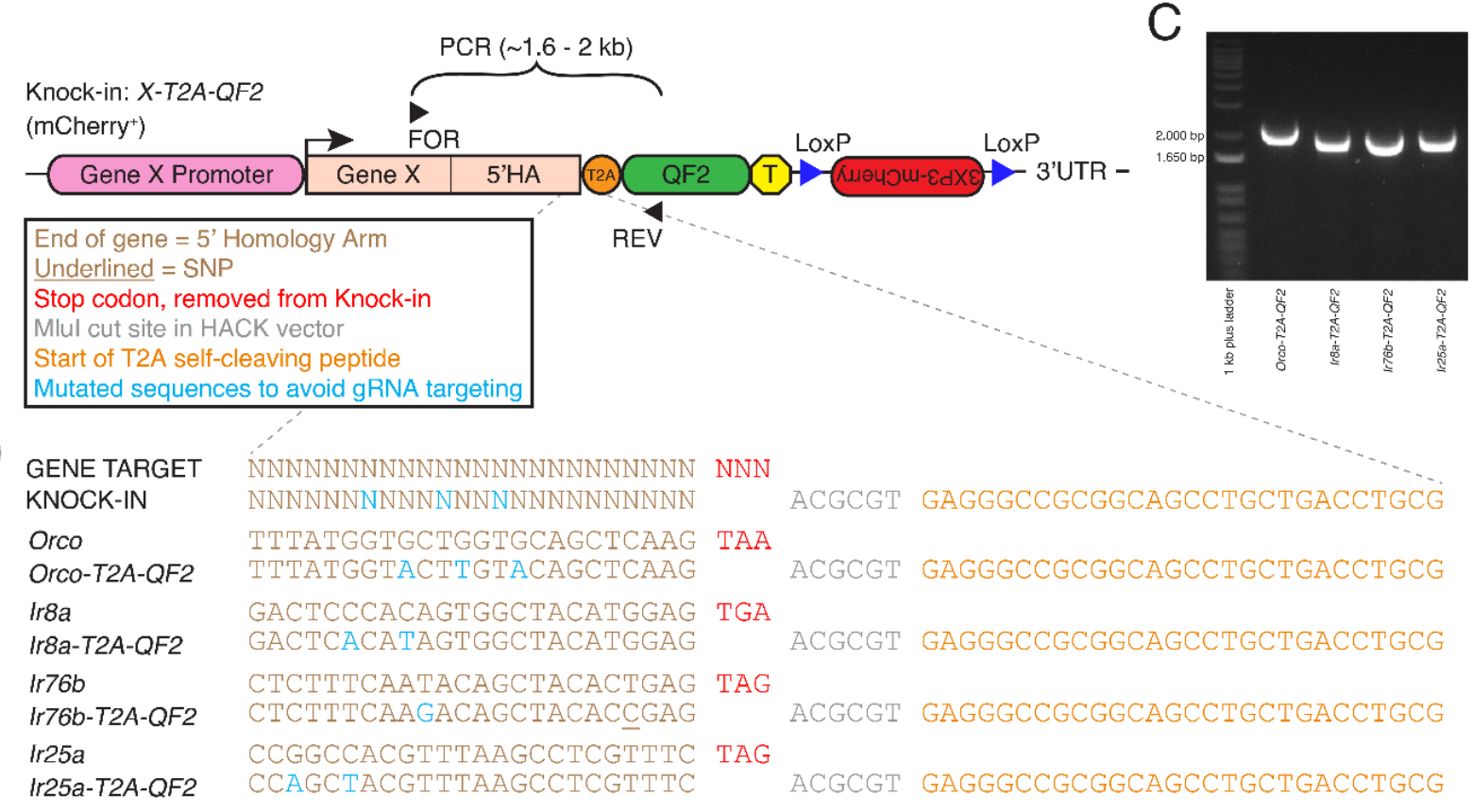

E

$\operatorname{Orco}^{2}$ mutant

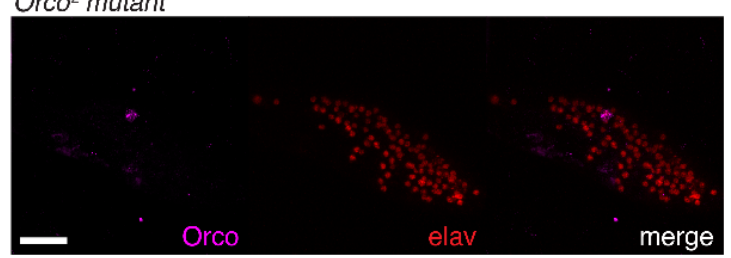

F

Ir8a mutant

$1 r 76 b^{1}$ mutant

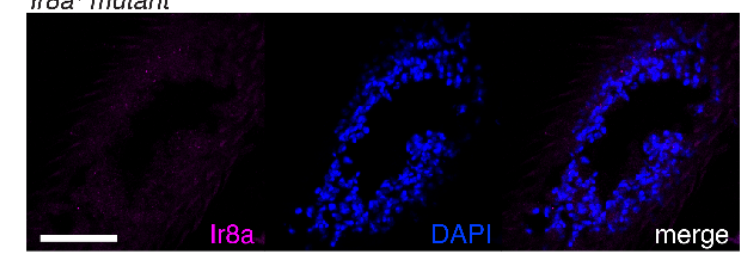

G

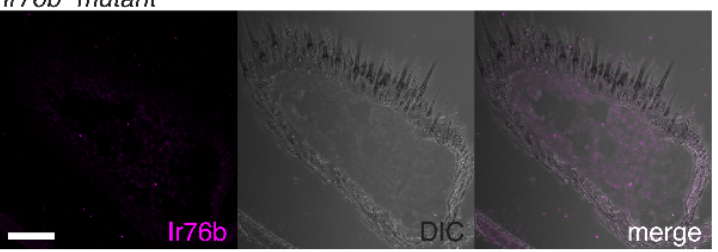

$\mathrm{H}$

Ir $25 a^{2}$ mutant

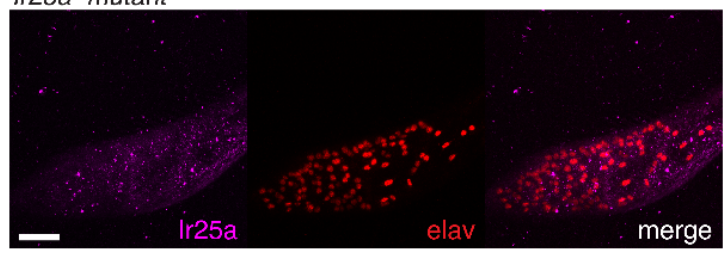


Figure 2-Figure Supplement 3. Additional Validation of Co-Receptor Knock-in Lines.

1080 A. The HACK 3XP3-mCherry marker is expressed in the eyes and broadly throughout the fly

1081 nervous system and is subject to positional effects in expression. B. Each knock-in line is 1082 generated by CRISPR-Cas9 mediated homology-directed insertion of a T2A-QF2 cassette 1083 marked by mCherry in front of the translational stop codon of the target gene. The 5' homology 1084 arm (5'HA) is $\sim 1 \mathrm{~kb}$ of the gene just before the stop codon. Successful knock-in lines were 1085 confirmed using forward primers upstream of the 5'HA (FOR), and a reverse primer within the 1086 QF2 sequence (REV). Amplification of a $\sim 1.6$ to $2 \mathrm{~kb}$ sequence occurs only if a knock-in is present. See Figure 2A for target and donor construct schematics. C. PCR genotyping using the primers shown in (B) confirms successful knock-in of the four targeted genes. D. Knock-ins were confirmed with sequencing of the targeted genomic site. For each co-receptor, the first line shows the target sequence (based on the FlyBase reference genome) (Thurmond et al., 2019), and the second line shows the verified sequence of the knock-in. The stop codon (red) has been replaced with the HACK T2A-QF2 cassette. The portion shown here includes the Mlul restriction site used for cloning the 5'HA (grey), as well as the start of the $T 2 A$ sequence

1094 (orange). In each knock-in construct, synonymous substitutions (blue) have been made to 1095 prevent the gRNA/Cas9 from cutting the donor construct. Each knock-in construct was designed 1096 with three substitutions. The underlined base pair in the Ir76b knock-in is a SNP discovered in 1097 the lab wildtype stock and considered when designing the knock-in construct. E.-H. Antibody 1098 and in situ probe validation. E. No anti-Orco expression in $\operatorname{Orco}^{2}$ mutant palps. elav is used as a 1099 pan-neuronal counterstain. F. No anti-Ir8a expression in $\operatorname{Ir} 8 a^{1}$ mutant antennae. DAPI is used as 1100 a cellular counterstain. G. No Ir76b probe signal in $I r 76 b^{1}$ mutant antennae. DIC used to 1101 visualize tissue. H. No anti-Ir25a expression in $/ r 25 a^{2}$ mutant palps (elav counterstain). Scale 1102 bars $=25 \mu \mathrm{m}$. See also Table 2. 

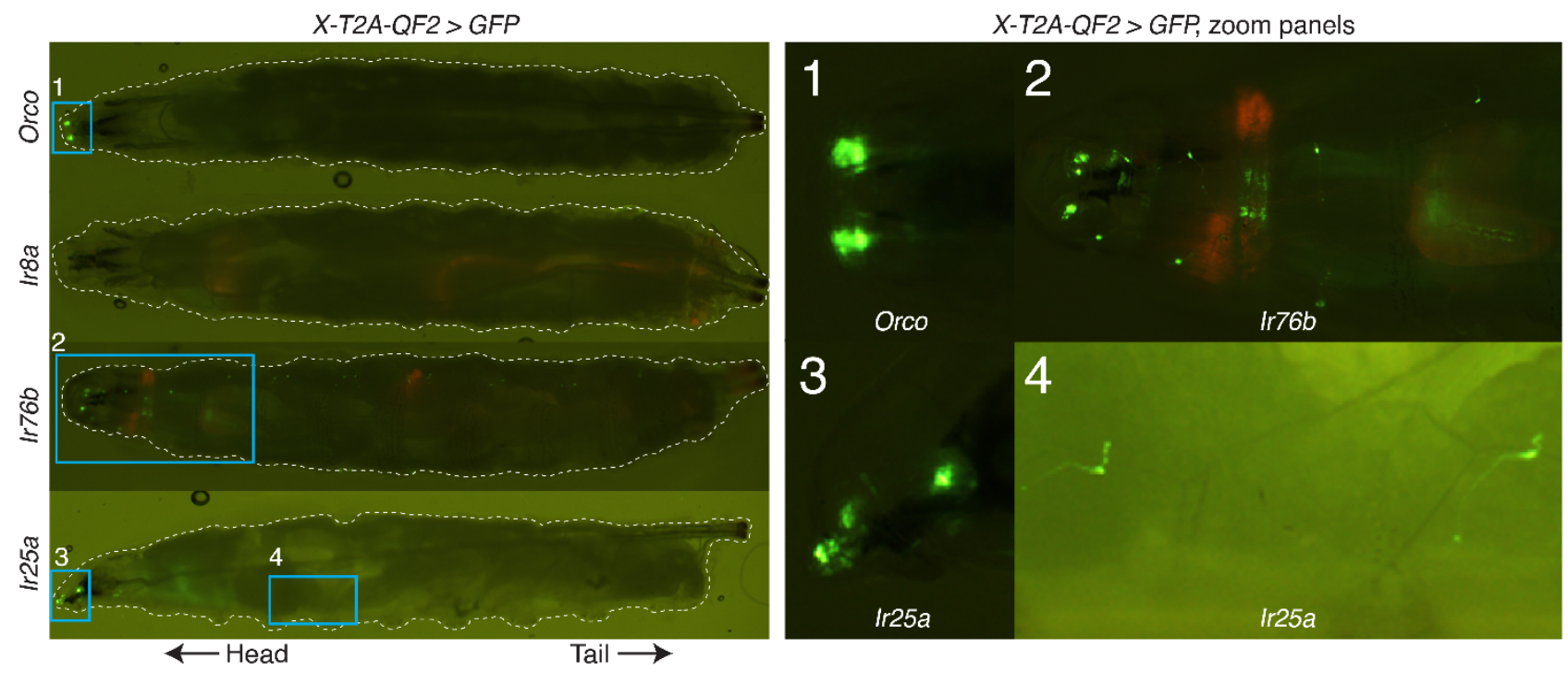

1104 Figure 2-Figure Supplement 4. Knock-in Expression in the Larva.

1105 In the larval stage, Orco-T2A-QF2 (first row) appears to drive expression only in the dorsal

1106 organs (zoom panel 1), as previously described (Larsson et al., 2004); Ir8a-T2A-QF2 (second

1107 row) does not appear to drive GFP expression in the larva (although very weak expression may

1108 not be visible at this magnification); and both Ir76b-T2A-QF2 (third row) and Ir25a-T2A-QF2

1109 (fourth row) drive GFP expression in the larval head as well as throughout the body wall (zoom

1110 panel 2 for $\operatorname{Ir} 76 b$; zoom panels 3 and 4 for Ir25a), reflecting the role of these genes in additional,

1111 non-olfactory modalities. Weak red fluorescence from the 3XP3-mCherry knock-in marker can

1112 be detected in the Ir8a-T2A-QF2 and Ir76b-T2A-QF2 larvae. This is due to channel bleed-

1113 through and is not seen in the other knock-in lines (Orco-T2A-QF2 and Ir25a-T2A-QF2) that

1114 have had the marker removed (see Methods for details). 

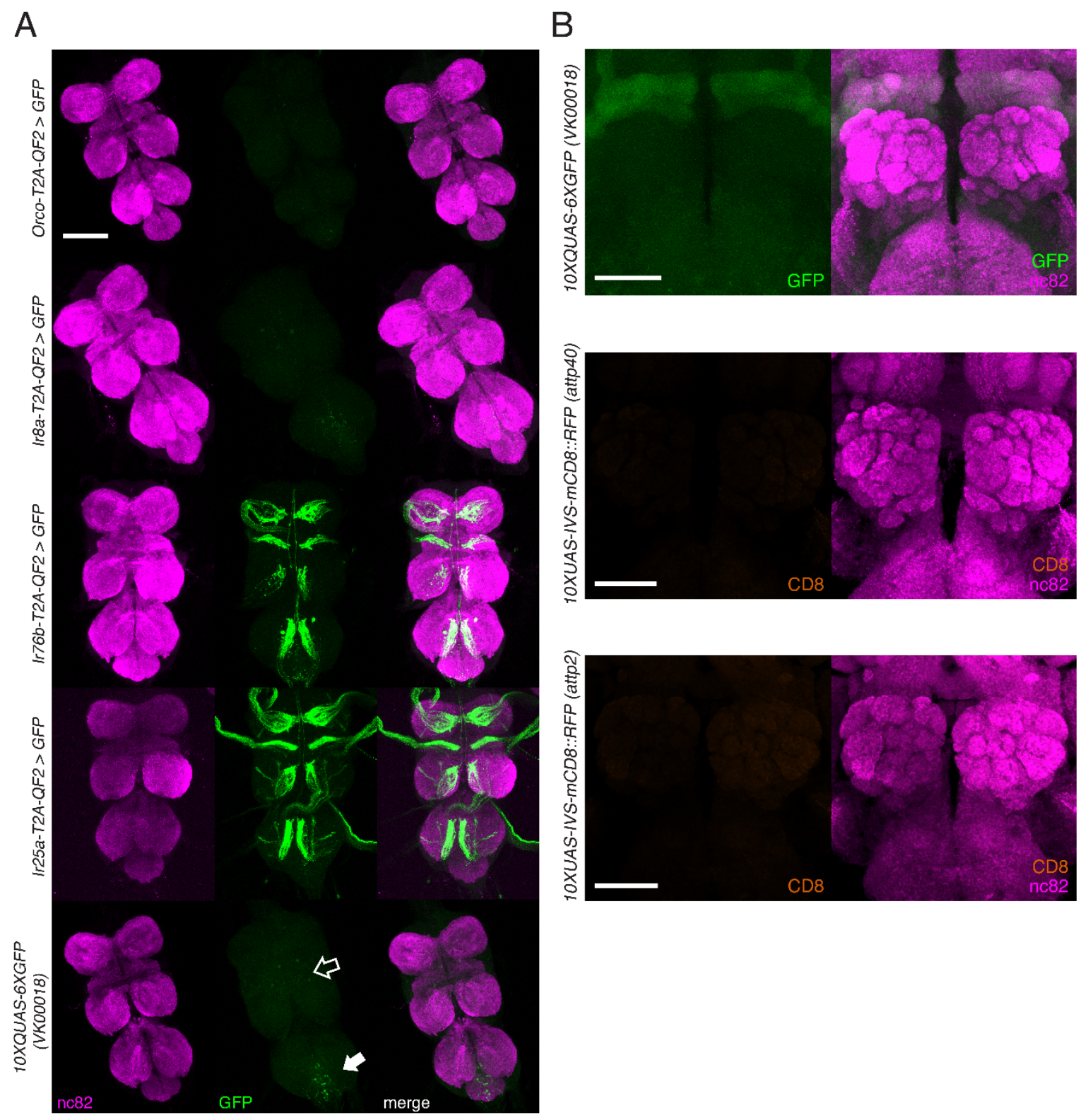

Figure 3-Figure Supplement 1. Knock-in Expression in the Adult Ventral Nerve Cord and

\section{Reporter Expression in the Brain.}

1118 A. In adult flies, two of the four knock-ins (Ir76b-T2A-QF2, third row, and Ir25a-T2A-QF2, fourth

1119 row) drive GFP expression in neurons innervating the ventral nerve cord (VNC). This likely

1120 reflects the role of these co-receptors in gustation. There is weak GFP expression in the

1121 reporter control (bottom row) in the abdominal neuromere (filled arrow), as well as the

1122 accessory mesothoracic neuropil (empty arrow), which can be seen in the two other knock-ins 
1123 (Orco-T2A-QF2, first row, and Ir8a-T2A-QF2, second row). This non-specific expression is likely

1124 from the reporter itself rather than driven by the two knock-ins. B. Control brains showing the

1125 QUAS-GFP reporter alone (top) and the UAS-mCD8::RFP reporters alone (middle and bottom).

1126 These reporters were used for all brain images in Figures $3-5$. The QUAS reporter weakly

1127 labels the mushroom bodies. The ventral nerve cord from the fly in (A, fourth row) is also used

1128 in Figure 2-Figure Supplement 1E. Scale bars: $100 \mu \mathrm{m}$ in (A), $50 \mu \mathrm{m}$ in (B). 
Figure 3-Figure Supplement 2

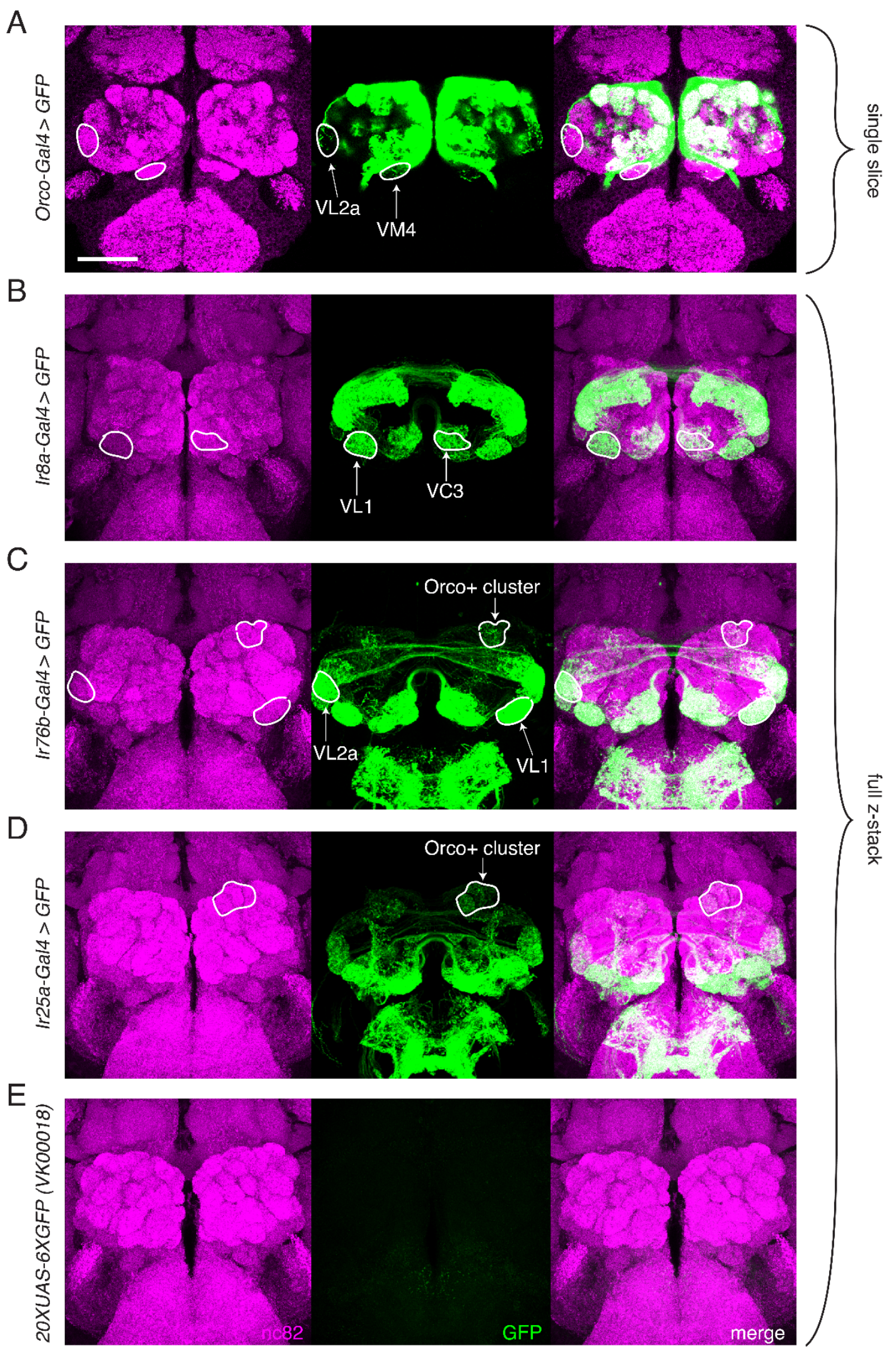


1130 Figure 3-Figure Supplement 2. Transgenic Co-Receptor Gal4 Lines Do Not Fully

1131 Recapitulate Knock-in Expression.

1132 A.-E. Transgenic co-receptor Gal4 lines crossed to a strong UAS reporter reveal expression in 1133 some, but not all, of the additional glomeruli labeled by the respective knock-ins. Some

1134 discrepancies between the Gal4 and knock-in lines are enumerated here, but do not represent a

1135 comprehensive list. A. Orco-Gal4 drives weak GFP expression in VL2a and VM4 glomeruli

1136 (outlined), but lacks expression in DL2, V, and VL1 glomeruli, which are labeled by the knock-in.

1137 B. Ir8a-Gal4>GFP labels VC3 and VL1 (outlined), but lacks expression in the VM4 and VA3

1138 glomeruli, which are labeled in the knock-in. C. Ir76b-Gal4 labels the VL1 and VL2a glomeruli

1139 (outlined), but lacks expression in the VA5 and DC3 glomeruli which are consistently labeled in

1140 the knock-in. Additionally, it drives GFP expression in Orco+ glomeruli not seen in the knock-in

1141 (outlined). D. Ir25a-Gal4 labels some Orco+ glomeruli (outline includes DA2, DA3, DA4m, and

1142 DA4I), but is lacking expression in many glomeruli labeled by the knock-in. E. The UAS-GFP

1143 control line has weak leaky expression in the SEZ but no expression in the AL. (A) shows single

1144 slice to visualize weakly labeled glomeruli; (B-E) are maximum intensity projections of z-stacks.

1145 Scale bar $=50 \mu \mathrm{m}$. See also Figure 3-Source Data 1. 


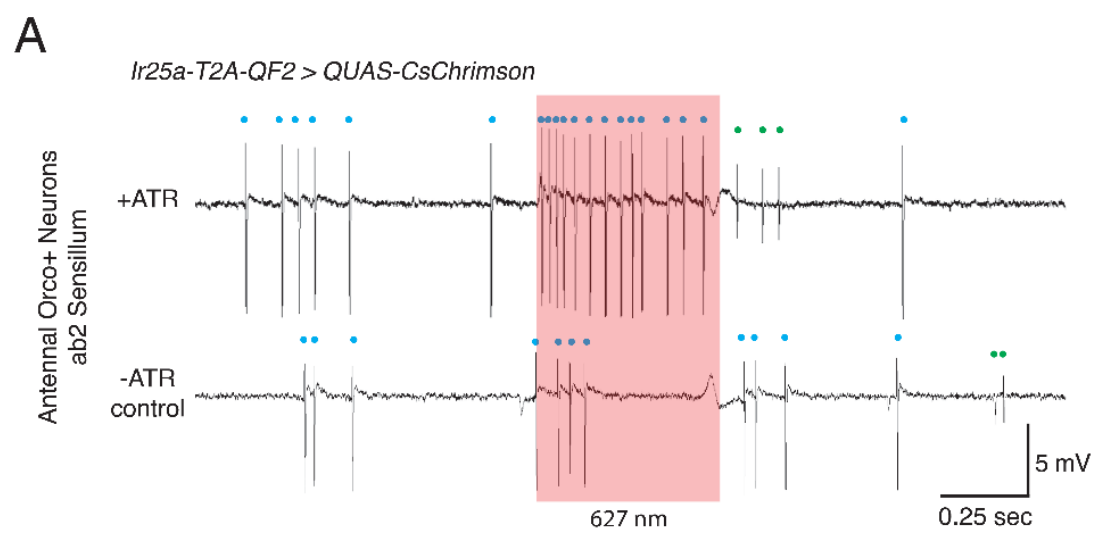

B
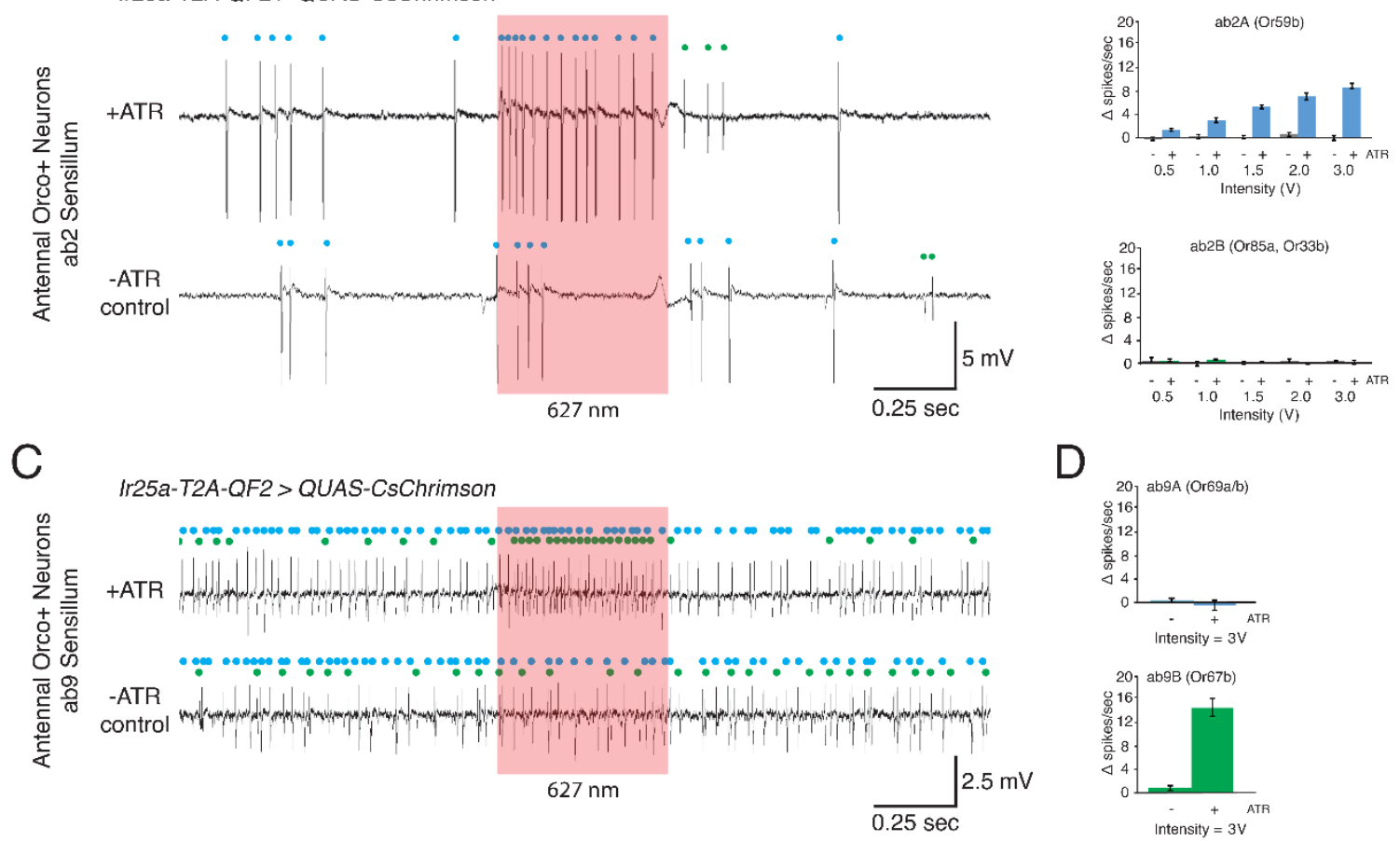

$\mathrm{D}$
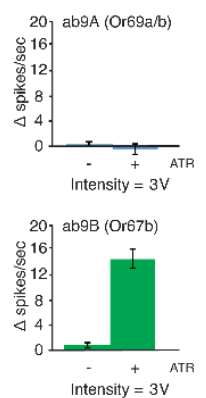

Figure 4-Figure Supplement 1. Optogenetic Experiments to Examine Ir25a Expression in

\section{Orco+ Neurons.}

1150 A-D. Confirmation of Ir25a expression in ab2 and ab9 sensilla using optogenetic stimulation.

1151 CsChrimson expression is driven by Ir25a-T2A-QF2, and recordings are performed in neurons

1152 previously determined to express only Orco. A. Representative SSR traces from ab2 using 1.5V

1153 of a $627 \mathrm{~nm}$ LED light (red box) to activate CsChrimson. Bottom trace is control animal which

1154 was not fed the necessary cofactor all-trans retinal (-ATR). ab2 has two neurons: blue dots

1155 indicate A neuron spikes, while green dots indicate B neuron spikes (genotype: Ir25a-T2A-QF2;

1156 QUAS-CsChrimson). B. Quantification of neuronal activity in response to light stimulation at

1157 various intensities ( $\mathrm{N}=7-12)$. Optogenetic experiments confirm Ir25a expression in ab2A

1158 (expressing Or59b, top; DM4 glomerulus) but not ab2B (expressing Or85a and Or33b, bottom;

1159 DM5 glomerulus). C. Combination of optogenetics and fluorescent guided SSR (Lin and Potter, 11602015 ) to examine Ir25a expression in ab9 sensilla (genotype: Ir25a-T2A-QF2; QUAS- 
1161 CsChrimson/Or67b-Gal4, 15XUAS-IVS-mcd8::GFP). Representative traces from ab9 in

1162 response to $3 \mathrm{~V}$ of $627 \mathrm{~nm}$ LED light (red box). As in (A), -ATR indicates the inactive

1163 CsChrimson control; blue dots indicate A neuron spikes, green dots indicate B neuron. D.

1164 Quantification of activity in response to light stimulation verified that Ir25a is expressed in ab9B

1165 (Or67b, bottom; VA3 glomerulus) but not in ab9A neurons (Or69aA/aB, top; D glomerulus). $\mathrm{N}=$ 11665.

1167 


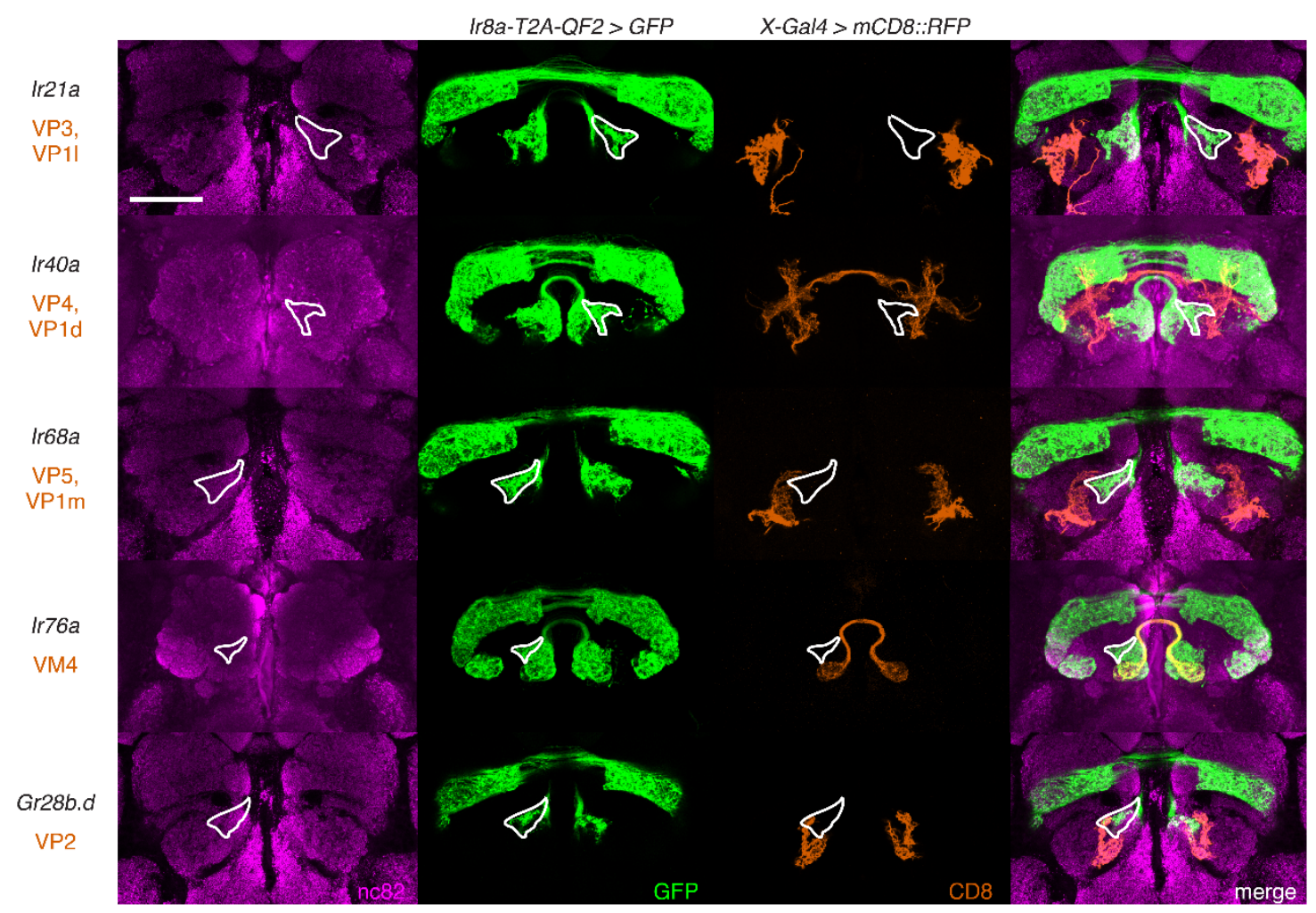

1169 Figure 5-Figure Supplement 1. The New Glomerular Region Labeled by the Ir8a Knock-in

1170 Does Not Correspond to Previously Identified Posterior Glomeruli.

1171 Co-labeling experiments with Ir8a-T2A-QF2 > GFP (green) and various Gal4 lines driving

$1172 m C D 8:: R F P$ (orange) confirm that the outlined region does not correspond to any of the VP

1173 glomeruli nor to VM4. Scale bar: $50 \mu \mathrm{m}$. 

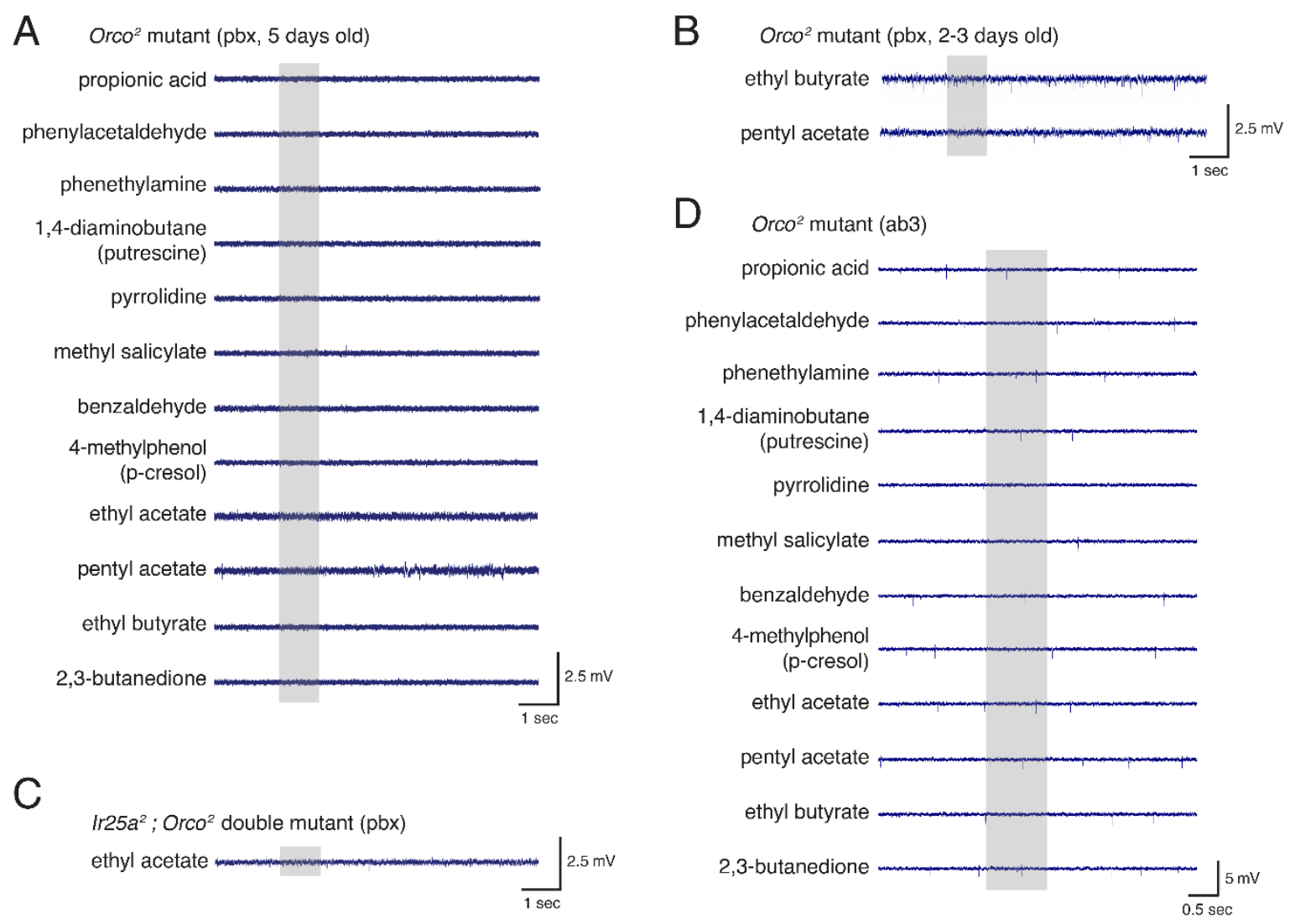

1175 Figure 6-Figure Supplement 1. Electrophysiological Experiments to Examine Ir25a

1176 Function in Orco+ Neurons.

1177 A. SSR recordings in $\operatorname{Orco}^{2}$ mutant palpal sensilla show neither baseline nor odor-evoked

1178 activity in response to a panel of odorants in 5-day old flies. $\mathrm{N}=20$ sensilla, 4 animals ( 3 male,

11791 female). B. Young Orco $^{2}$ mutant palpal neurons also do not have spontaneous or odor-evoked

1180 activity. C. Ir25a ${ }^{2}$; Orco ${ }^{2}$ double mutant palpal sensilla show neither baseline nor odor-evoked

1181 activity. $\mathrm{N}=42$ sensilla from 5 animals (3 male, 2 female). D. Antennal ab3 sensilla do

1182 occasionally show baseline activity in the $\operatorname{Orco}^{2}$ mutant, but generally do not show odor-evoked

1183 activity ( $N=5$ flies; see also Figure 6). Grey box indicates the time of stimulus delivery. See

1184 also Figure 6-Source Data 1. 
1185 Figure 6-Figure Supplement 2

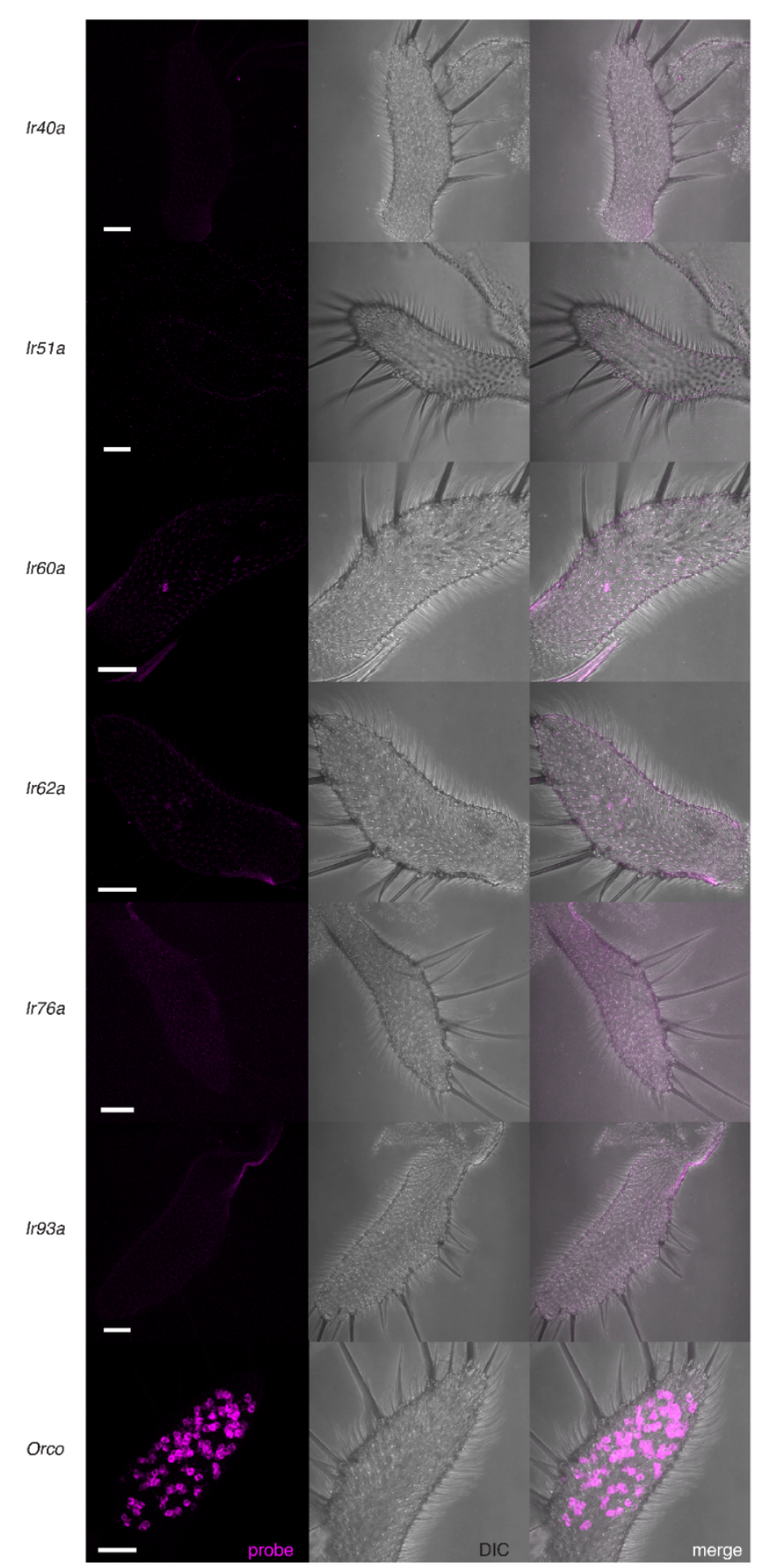

1186 Figure 6-Figure Supplement 2. No IrX Expression of the Top Candidates in the Maxillary

1187 Palps.

1188 The recent fly cell atlas (Li et al., 2021) was used to identify potential IrX tuning receptors in the 1189 palps. In situ experiments did not reveal strong expression of any of these target genes. Orco 1190 (bottom) was used as a positive control. See also Figure 6-Source Data 2. Scale bars $=25 \mu \mathrm{m}$. 


\section{Materials and Methods}

1192 Key Resources Table

\begin{tabular}{|c|c|c|}
\hline REAGENT or RESOURCE & SOURCE & IDENTIFIER \\
\hline \multicolumn{3}{|l|}{ Antibodies } \\
\hline Mouse anti-nc82 & DSHB & $\begin{array}{l}\text { Cat\# nc82; RRID: } \\
\text { AB_2314866 }\end{array}$ \\
\hline Rat anti-cd8 & Thermo Fisher Scientific & $\begin{array}{l}\text { Cat\# 14-0081-82; } \\
\text { RRID: AB 467087 }\end{array}$ \\
\hline Rat anti-elav & DSHB & $\begin{array}{l}\text { Cat\# Rat-Elav- } \\
\text { 7E8A10; RRID: } \\
\text { AB 528218 }\end{array}$ \\
\hline Rabbit anti-Orco & $\begin{array}{l}\text { Gift from Leslie Vosshall; } \\
\text { (Larsson et al., 2004) }\end{array}$ & $\mathrm{N} / \bar{A}$ \\
\hline Rabbit anti-Ir25a & $\begin{array}{l}\text { Gift from Richard Benton; } \\
\text { (Benton et al., 2009) }\end{array}$ & RRID: AB_2567027 \\
\hline Guinea pig anti-Ir8a & $\begin{array}{l}\text { Gift from Richard Benton; } \\
\text { (Abuin et al., 2011) }\end{array}$ & RRID:AB_2566833 \\
\hline Rabbit anti-Ir64a & $\begin{array}{l}\text { Gift from Greg Suh; (Ai et } \\
\text { al., 2010) }\end{array}$ & RRID:AB_2566854 \\
\hline Goat anti-guinea pig Alexa 568 & Thermo Fisher Scientific & $\begin{array}{l}\text { Cat\# A11075; RRID: } \\
\text { AB_141954 }\end{array}$ \\
\hline Goat anti-rabbit Cy3 conjugated AffiniPure 568 & Jackson ImmunoResearch & $\begin{array}{l}\text { Cat\# 111-165-144; } \\
\text { RRID: AB_2338006 }\end{array}$ \\
\hline Goat anti-mouse Cy3 & Jackson ImmunoResearch & $\begin{array}{l}\text { Cat\# 115-165-166; } \\
\text { RRID: AB 2338692 }\end{array}$ \\
\hline Goat anti-mouse Alexa 647 & Jackson ImmunoResearch & $\begin{array}{l}\text { Cat\# 115-605-166; } \\
\text { RRID: AB_2338914 }\end{array}$ \\
\hline Donkey anti-guinea pig Cy3 & Jackson ImmunoResearch & $\begin{array}{l}\text { Cat\# 706-165-148; } \\
\text { RRID: AB_2340460 }\end{array}$ \\
\hline Goat anti-rat Cy3 & Jackson ImmunoResearch & $\begin{array}{l}\text { Cat\# 112-165-167; } \\
\text { RRID: AB_2338251 }\end{array}$ \\
\hline Goat anti-rat Alexa 647 & Jackson ImmunoResearch & $\begin{array}{l}\text { Cat\# 112-605-167; } \\
\text { RRID: AB_2338404 }\end{array}$ \\
\hline Goat anti-rabbit Cy3 & Jackson ImmunoResearch & $\begin{array}{l}\text { Cat\# 111-165-144; } \\
\text { RRID: AB_2338006 }\end{array}$ \\
\hline Goat anti-rabbit Alexa 647 & Jackson ImmunoResearch & $\begin{array}{l}\text { Cat\# 111-605-144; } \\
\text { RRID: AB_2338078 }\end{array}$ \\
\hline \multicolumn{3}{|l|}{ Chemicals, Peptides, and Recombinant Proteins } \\
\hline Mineral oil & Sigma-Aldrich & CAS\# 8042-47-5 \\
\hline Ethyl acetate & Sigma-Aldrich & CAS\# 141-78-6 \\
\hline Pentyl acetate & Sigma-Aldrich & CAS\# 628-63-7 \\
\hline Benzaldehyde & Sigma-Aldrich & CAS\# 100-52-7 \\
\hline Ethyl butyrate & Sigma-Aldrich & CAS\# 105-54-4 \\
\hline Hexanol & Sigma-Aldrich & CAS\# 111-27-3 \\
\hline E2-hexenal & Sigma-Aldrich & CAS\# 6728-26-3 \\
\hline Geranyl acetate & Sigma-Aldrich & CAS\# 105-87-3 \\
\hline 2-heptanone & Sigma-Aldrich & CAS\# 110-43-0 \\
\hline 1-octen-3-ol & Sigma-Aldrich & CAS\# 3391-86-4 \\
\hline
\end{tabular}




\begin{tabular}{|c|c|c|}
\hline 2,3-butanedione & Sigma-Aldrich & CAS\# 431-03-8 \\
\hline Phenylacetaldehyde & Sigma-Aldrich & CAS\# 122-78-1 \\
\hline Phenethylamine & Sigma-Aldrich & CAS\# 64-04-0 \\
\hline Propionic acid & Sigma-Aldrich & CAS\# 79-09-4 \\
\hline 1,4-diaminobutane & Sigma-Aldrich & CAS\# 110-60-1 \\
\hline Pyrrolidine & Sigma-Aldrich & CAS\# 123-75-1 \\
\hline P-cresol & Sigma-Aldrich & CAS\# 106-44-5 \\
\hline Methyl salicylate & Sigma-Aldrich & CAS\# 119-36-8 \\
\hline Paraffin Oil & ACROS Organics & CAS\# 8012-95-1 \\
\hline Water & SIGMA Life Science & CAS\# 7732-18-5 \\
\hline Acetic acid & Fisher Scientific & CAS\# 64-19-7 \\
\hline Ammonium hydroxide & Fisher Scientific & CAS\# 7664-41-7 \\
\hline Cadaverine & Sigma-Aldrich & CAS\# 462-94-2 \\
\hline Hexanol & ACROS Organics & CAS\# 111-27-3 \\
\hline 2,3-butanedione & ACROS Organics & CAS\# 431-03-8 \\
\hline Phenethylamine & ACROS Organics & CAS\# 64-04-0 \\
\hline Propanal & ACROS Organics & CAS\# 123-38-6 \\
\hline 1,4-diaminobutane & ACROS Organics & CAS\# 110-60-1 \\
\hline All trans-retinal & Sigma-Aldrich & CAS\# 116-31-4 \\
\hline \multicolumn{3}{|l|}{ Critical Commercial Assays } \\
\hline HCR v3.0 & Molecular Instruments & $\mathrm{N} / \mathrm{A}$ \\
\hline In-Fusion Cloning & Clontech Labs & Cat\# 639645 \\
\hline DNeasy Blood and Tissue Kit & Qiagen & Cat\# 69506 \\
\hline \multicolumn{3}{|l|}{ Deposited Data } \\
\hline Raw and analyzed data & This paper & $\begin{array}{l}\text { Available upon } \\
\text { request }\end{array}$ \\
\hline snRNAseq data & (McLaughlin et al., 2021) & $\begin{array}{l}\text { GSE162121; } \\
\text { https://github.com/co } \\
\text { lleen- } \\
\text { mclaughlin/ORN se } \\
\text { q/ }\end{array}$ \\
\hline EM reconstructions & This paper & $\begin{array}{l}\text { https://flywire.ai/\#link } \\
\text { s/Task2021a/all }\end{array}$ \\
\hline \multicolumn{3}{|l|}{ Experimental Models: Organisms/Strains } \\
\hline D. melanogaster: Orco-T2A-QF2 knock-in & This paper & $\begin{array}{l}\text { BDSC: } 92400, \\
92401,92402\end{array}$ \\
\hline D. melanogaster. Ir8a-T2A-QF2 knock-in & This paper & $\begin{array}{l}\text { BDSC: } 92398, \\
92399\end{array}$ \\
\hline D. melanogaster: Ir76b-T2A-QF2 knock-in & This paper & $\begin{array}{l}\text { BDSC: } 92396, \\
92397\end{array}$ \\
\hline D. melanogaster: Ir25a-T2A-QF2 knock-in & This paper & $\begin{array}{l}\text { BDSC: } 92392, \\
\text { 92393, } 92394, \\
92395\end{array}$ \\
\hline $\begin{array}{l}\text { D. melanogaster: } y[1] w\left[{ }^{\star}\right] ; \text { Pin[1]/CyO; } \\
P(w[+m C]=10 X Q U A S-C s C h r i m s o n . m V e n u s) 11 c\end{array}$ & This paper & $\begin{array}{l}\text { BDSC: } 91996 \\
\text { FlyBase: } \\
\text { FBst0091996 }\end{array}$ \\
\hline D. melanogaster: Ir21a-T2A-Gal4 knock-in & $\begin{array}{l}\text { Gift from Paul Garrity; } \\
\text { (Marin et al., 2020) }\end{array}$ & N/A \\
\hline D. melanogaster: Ir68a-T2A-Gal4 knock-in & $\begin{array}{l}\text { Gift from Paul Garrity; } \\
\text { (Marin et al., 2020) }\end{array}$ & $\mathrm{N} / \mathrm{A}$ \\
\hline
\end{tabular}




\begin{abstract}
D. melanogaster: Ir64a-Gal4
D. melanogaster: Rh50-Gal4

D. melanogaster: Amt-Gal4

D. melanogaster: Repo-Gal80

D. melanogaster: wCS (Cantonized $w^{1118}$ )

D. melanogaster: Or35a-Gal4

D. melanogaster: $y[1] w[67 c 23]$

$P(y[+m D i n t 2]=C r e y) 1 b ;$ sna[Sco]/CyO

D. melanogaster: $\left.w^{*}\right] ;$ I(2) ${ }^{*}\left[{ }^{*}\right] / C y O$;

$P(w[+m C]=O r 10 a-G A L 4 . C) 134 t 1.3$

D. melanogaster: $w\left[^{*}\right] ; P(w[+m C]=O r 22 a-$ GAL4.7.717)14.2
\end{abstract}

D. melanogaster: $w\left[{ }^{*}\right] ; P(w[+m C]=O r 22 a-$ GAL4.7.717)14.21

D. melanogaster: $w[*] ; P(w[+m C]=0 r 33 c-$ GAL4.F)78.3

D. melanogaster: $w[*] ; P(w[+m C]=O r 35 a-$ GAL4.F)109.2A

D. melanogaster: $\left.w{ }^{\star}\right] ; P(w[+m C]=0 r 35 a-$ GAL4.F)109.3

D. melanogaster: $w[*] ; P(w[+m C]=0 r 42 a-$ GAL4.F) $48.3 B$

D. melanogaster: $\left.w{ }^{*}\right] ; P(w[+m C]=O r 42 b-$ GAL4.F)64.3

D. melanogaster: $w\left[{ }^{\star}\right] ; P(w[+m C]=O r 47 a-$ GAL4.8.239)15.4A

D. melanogaster: $w[*] ; P(w[+m C]=O r 49 b-$ GAL4.F)80.1

D. melanogaster: $w\left[^{*}\right] ; B I[1] / S M 1 ; P(w[+m C]=O r 65 a-$ GAL4.F)72.1

D. melanogaster: $w[*] ; P(w[+m C]=O r 67 b-$ GAL4.F)68.3/TM6B, $T b[1]$

D. melanogaster: $w[*] ; P(w[+m C]=O r 71 a-$ GAL4.F)30.4

D. melanogaster: $w\left[{ }^{\star}\right] ; P(w[+m C]=O r 83 c-$ GAL4.F)73.3B
Gift from Greg Suh lab; (Ai N/A et al., 2010)

(Vulpe et al., 2021a) N/A

(Menuz et al., 2014)

(Awasaki et al., 2011)

(Koh et al., 2014)

(Yao et al., 2005)

N/A

FlyBase:

FBtp0067904

FlyBase:

FBrf0226011

N/A

Bloomington Drosophila

Stock Center

Bloomington Drosophila

Stock Center

Bloomington Drosophila

Stock Center

Bloomington Drosophila

Stock Center

Bloomington Drosophila Stock Center

Bloomington Drosophila

Stock Center

Bloomington Drosophila Stock Center

Bloomington Drosophila Stock Center

Bloomington Drosophila Stock Center

Bloomington Drosophila Stock Center

Bloomington Drosophila

Stock Center

Bloomington Drosophila Stock Center

Bloomington Drosophila Stock Center

Bloomington Drosophila Stock Center

Bloomington Drosophila Stock Center
BDSC: 766;

FlyBase:

FBti0012692

BDSC: 23885;

FlyBase:

FBti0102042

BDSC: 9951;

FlyBase:

FBti0101805

BDSC: 9952;

FlyBase:

FBti0101805

BDSC: 9966;

FlyBase:

FBti0101843

BDSC: 9967;

FlyBase:

FBti0101810

BDSC: 9968;

FlyBase:

FBti0101844

BDSC: 9970;

FlyBase:

FBti0101811

BDSC: 9971;

FlyBase:

FBti0101812

BDSC: 9982;

FlyBase:

FBti0101851

BDSC: 9986;

FlyBase:

FBti0101853

BDSC: 9994;

FlyBase:

FBti0101857

BDSC: 9995;

FlyBase:

FBti0101858

BDSC: 23122;

FlyBase:

FBti0101860

BDSC: 23131;

FlyBase:

FBti0101829 
D. melanogaster: $w[*] ; P(w[+m C]=O r 85 a-$ GAL4.F)67.2

D. melanogaster: $w[*] ; P(w[+m C]=O r 92 a-$ GAL4.F)62.1

D. melanogaster: $w[*] ; P(w[+m C]=O r 98 a-$ GAL4.F)115.1

D. melanogaster: $w[1118] ; P(w[+m C]=O r 46 a-$ GAL4.G)32.1.y

D. melanogaster: $w\left[{ }^{\star}\right] ; P(w[+m C]=O r 13 a-$ GAL4.C)229t56.2/TM3, Sb[1]

D. melanogaster: $w[*] ; P(w[+m C]=O r 43 b-$ GAL4.C)110t8.1

D. melanogaster: $w[*] ; P(w[+m C]=O r 59 b-$ GAL4.C)114t2.2

D. melanogaster: $w\left[^{\star}\right] ; P(w[+m C]=O r 59 c-$ GAL4.C)129t1.1

D. melanogaster: $w[*] ; P(w[+m C]=O r 67 a-$ GAL4.C)137t3.3

D. melanogaster: $w\left[{ }^{\star}\right] ; P(w[+m C]=O r 67 c-$ GAL4.C)116t3.2/CyO

D. melanogaster: $w[*] ; P(w[+m C]=O r 85 b-$ GAL4.C) $179 t 5.1$

D. melanogaster: $w[*] ; P(w[+m C]=O r 85 d-$ GAL4.C)143t2.1

D. melanogaster: $\left.w{ }^{*}\right] ; P(w[+m C]=$ OrcoGAL4.W)11.17; TM2/TM6B, Tb[1]

D. melanogaster: $\left.w{ }^{*}\right] ; P(w[+m C]=I r 40 a-$ GAL4.3011)214.1; TM2/TM6B, Tb[1]

D. melanogaster: $w\left[^{*}\right] ; P(w[+m C]=I r 25 a-$ GAL4.A)236.1; TM2/TM6B, Tb[1]

D. melanogaster: $w\left[{ }^{*}\right] ; P(w[+m C]=I r 76 b-$ GAL4.916)226.8; TM2/TM6B, Tb[+]

D. melanogaster: $w\left[{ }^{\star}\right] ; P(w[+m C]=I r 8 a-$ GAL4.A)204.8; TM2/TM6B, Tb[1]

D. melanogaster: $w\left[{ }^{*}\right] ; P(w[+m C]=I r 64 a-$ GAL4.A)183.8; TM2/TM6B, Tb[1]
Bloomington Drosophila Stock Center

Bloomington Drosophila Stock Center

Bloomington Drosophila Stock Center

Bloomington Drosophila Stock Center

Bloomington Drosophila Stock Center

Bloomington Drosophila Stock Center

Bloomington Drosophila Stock Center

Bloomington Drosophila Stock Center

Bloomington Drosophila Stock Center

Bloomington Drosophila Stock Center

Bloomington Drosophila Stock Center

Bloomington Drosophila Stock Center

Bloomington Drosophila Stock Center

Bloomington Drosophila Stock Center

Bloomington Drosophila Stock Center

Bloomington Drosophila Stock Center

Bloomington Drosophila Stock Center

Bloomington Drosophila Stock Center
BDSC: 23133;

FlyBase:

FBti0101830

BDSC: 23139;

FlyBase:

FBti0101867

BDSC: 23141;

FlyBase:

FBti0101868

BDSC: 23291;

FlyBase:

FBti0076800

BDSC: 23886;

FlyBase:

FBti0102056

BDSC: 23894;

FlyBase:

FBti0102047

BDSC: 23897;

FlyBase:

FBti0102060

BDSC: 23899;

FlyBase:

FBti0102061

BDSC: 23904;

FlyBase:

FBti0102049

BDSC: 23905;

FlyBase:

FBti0102050

BDSC: 23911;

FlyBase:

FBti0102053

BDSC: 24148;

FlyBase:

FBti0102066

BDSC: 26818;

FlyBase:

FBti0101150

BDSC: 41727;

FlyBase:

FBst0041727

BDSC: 41728;

FlyBase:

FBti0148895

BDSC: 41730;

FlyBase:

FBti0153291

BDSC: 41731;

FlyBase:

FBti0148897

BDSC: 41732;

FlyBase:

FBti0148898 
D. melanogaster: $w\left[{ }^{\star}\right] ; P(w[+m C]=I r 76 a-$ GAL4.PB)292.3B; TM2/TM6B, Tb[1]

D. melanogaster: $w[*] ; P(y[+t 7.7] w[+m C]=I r 41 a-$ GAL4.2474)attP40; TM2/TM6B, Tb[1]

D. melanogaster: $w[*] ; P(w[+m C]=0 r 35 a-$ MmusiCd8a.GFP)3/TM3, Sb[1]

D. melanogaster: $w[*] ; P(w[+m C]=G r 21 a-$ GAL4.9.323)2/CyO; $\operatorname{Dr}[1] / T M 3, S b[+]$

D. melanogaster: $w\left[^{*}\right] ; P(w[+m C]=G r 28 b . d-$ GAL4)B27; $\operatorname{Dr}[1] / T M 3, S b[1]$

D. melanogaster: $\left.y[1] w{ }^{*}\right]$ TI(GAL4)Or7a[KIGAL4.w-]

D. melanogaster: QUAS reporter: $y$ [1] w[1118]; $P(w[+m C]=Q U A S-m C D 8:: G F P . P) 5 B / T M 6 B, T b[1]$

D. melanogaster: QUAS reporter: $y[1] w\left[{ }^{*}\right]$; $P B a c(y[+m D i n t 2] w[+m C]=10 X Q U A S-$ 6XGFP)VK00018/CyO, P(Wee-P.ph0)Bacc[WeeP20]

D. melanogaster: UAS reporter: $w[*] ; P(y[+t 7.7]$ $w[+m C]=10 X U A S-I V S-m C D 8:: G F P)$ attP40

D. melanogaster: UAS reporter: $w\left[{ }^{*}\right] ; P(y[+t 7.7]$ $w[+m C]=15 X U A S-I V S-m C D 8:: G F P)$ attP2

D. melanogaster: UAS reporter: $w\left[{ }^{*}\right] ; P(y[+t 7.7]$ $w[+m C]=10 X U A S-I V S-m C D 8:: R F P)$ att $P 2$

D. melanogaster: UAS reporter: $w[*] ; P(y[+t 7.7]$ $w[+m C]=10 X U A S-I V S-m C D 8:: R F P)$ attP40

D. melanogaster: UAS reporter: $y[1] w\left[{ }^{*}\right]$; $P B a c(y[+m D i n t 2] w[+m C]=20 X U A S-$

6XGFP)VK00018/CyO, P(Wee-P.ph0)Bacc[Wee$P 20]$

D. melanogaster: UAS reporter (sacculus experiments): UAS- $m C D 8:: G F P\left(2^{\text {nd }}\right)$

$D$. melanogaster: UAS reporter (sacculus experiments): UAS-mCD8::GFP $\left(3^{\text {rd }}\right)$

D. melanogaster: $\operatorname{Orco}^{2}$ mutant: $w\left[{ }^{\star}\right]$; $\mathrm{TI}\left(w\left[+m^{*}\right]=\mathrm{TI}\right) \operatorname{Orco}[2]$

D. melanogaster: Ir $25 a^{2}$ mutant: $\left.w{ }^{\star}\right]$; $\mathrm{TI}\left(\mathrm{w}\left[+\mathrm{m}^{*}\right]=\mathrm{TI}\right) / \mathrm{r} 25 \mathrm{a}[2] / \mathrm{CyO}$

D. melanogaster: Ir8a ${ }^{1}$ mutant: $w\left[{ }^{*}\right]$ $T I(w[+m W . h s]=T I) I r 8 a[1] ; B I[1] L[2] / C y O$
Bloomington Drosophila Stock Center

Bloomington Drosophila Stock Center

Bloomington Drosophila Stock Center

Bloomington Drosophila Stock Center

Bloomington Drosophila Stock Center

Potter lab

Bloomington Drosophila Stock Center

Bloomington Drosophila Stock Center

Bloomington Drosophila Stock Center

Bloomington Drosophila Stock Center

Bloomington Drosophila Stock Center

Bloomington Drosophila Stock Center

Bloomington Drosophila Stock Center

(Lee and Luo, 1999)

(Lee and Luo, 1999)

Bloomington Drosophila Stock Center

Bloomington Drosophila Stock Center

Bloomington Drosophila Stock Center
BDSC: 41735;

FlyBase:

FBst0041735

BDSC: 41749;

FlyBase:

FBst0041749

BDSC: 52624;

FlyBase:

FBti0156834

BDSC: 57600;

FlyBase:

FBti0162643

BDSC: 57620;

FlyBase:

FBst0057620

BDSC: 91991;

FlyBase:

FBti0214362

BDSC: 30003 ;

FlyBase:

FBti0129937

BDSC: 52264;

FlyBase:

FBti0162759

BDSC: 32186 ;

FlyBase:

FBti0131963

BDSC: 32193;

FlyBase:

FBti0131935

BDSC: 32218;

FlyBase:

FBti0131950

BDSC: 32219;

FlyBase:

FBti0131967

BDSC: 52261;

FlyBase:

FBti0162758

FlyBase:

FBti0012685

FlyBase:

FBti0012686

BDSC: 23130;

FlyBase:

FBti0168777

BDSC: 41737;

FlyBase:

FBti0168524

BDSC: 41744;

FlyBase:

FBst0041744 


\author{
D. melanogaster: Ir76b ${ }^{1}$ mutant: $w\left[{ }^{\star}\right]$; Ir76b[1] \\ D. melanogaster: germline Cas9: $y[1]$ \\ $M(R F P[3 \times P 3 . P B]$ GFP[E.3xP3]=vas-Cas9)ZH-2A \\ $w[1118] / F M 7 c$ \\ D. melanogaster: germline Cas9: w[1118]; \\ PBac(y[+mDint2]=vas-Cas9)VK00027 \\ D. melanogaster: germline Cas9: $y[1]$ \\ $\left.M(w[+m C]=A c t 5 C-C a s 9 . P) Z H-2 A w{ }^{*}\right]$ \\ D. melanogaster: double balancer: 19ADrok/FM7c; \\ $\mathrm{Pin} / \mathrm{CyO}$ \\ D. melanogaster: double balancer: $y, w ; P i n / C y O$; \\ Dh/TM6B
}

D. melanogaster: double balancer: $y, w ; S / C y O ;$ Pr/TM6B

D. melanogaster: single balancer: $y, w ;+/+; \operatorname{Pr} / T M 6 B$

D. melanogaster: single balancer: $y, w ; S / C y O ;+/+$

D. melanogaster: wildtype: $w^{1118} / s o D 1$

\begin{tabular}{l} 
Oligonucleotides \\
\hline Orco_gRNA_FOR: \\
TCCGGGTGACTTCGCACAGTGCGGAGGGGGC \\
AAGTTTTAGAGCTAGAAATAGCAAGTTA \\
OrCo_gRNA_REV: \\
TTCTAGCTCTAAACACTTTATGGTGCTGGTGCA \\
GCGACGTTAAATTGAAATAGGTC \\
OrCO_5HA_FOR: \\
CCCTTACGTAACGCGTCAGCTTGTTTGACTTACT \\
TGATTAC \\
OrCO_5HA_REV: \\
CGCGGCCCTCACGCGTCTTGAGCTGTACAAGTA \\
CCATAAAGT \\
OrCO_3HA_FOR: \\
GTTATAGATCACTAGTCTCAGTACTATGCAACCA \\
GCAATA
\end{tabular}

Bloomington Drosophila Stock Center

Bloomington Drosophila Stock Center

Bloomington Drosophila Stock Center

Bloomington Drosophila Stock Center

N/A (Potter lab stock)

N/A (Potter lab stock)

N/A (Potter lab stock)

N/A (Potter lab stock)

N/A (Potter lab stock)

Gift from Thomas R. Clandinin

BDSC: 51309;

FlyBase:

FBst0051309

BDSC: 51323;

FlyBase:

FBti0154823

BDSC: 51324;

FlyBase:

FBti0154822

BDSC: 54590;

FlyBase:

FBti0159182

Derived from BDSC

6666, FBba0000009,

FBal0013831,

FBba0000025

Derived from

FBal0013831,

FBba0000025,

FBti0004009,

FBba0000057,

FBal0016730

Derived from

FBal0015108,

FBba0000025,

FBal0013944,

FBba0000057,

FBal0016730

Derived from

FBal0013944,

FBba0000057,

FBal0016730

Derived from

FBal0015108,

FBba0000025

Derived from

FBal0018186

Integrated DNA N/A

Technologies; This paper

Integrated DNA

N/A

Technologies; This paper

Integrated DNA

N/A

Technologies; This paper

Integrated DNA

N/A

Technologies; This paper

Integrated DNA

N/A

Technologies; This paper
$\mathrm{N} / \mathrm{A}$ 


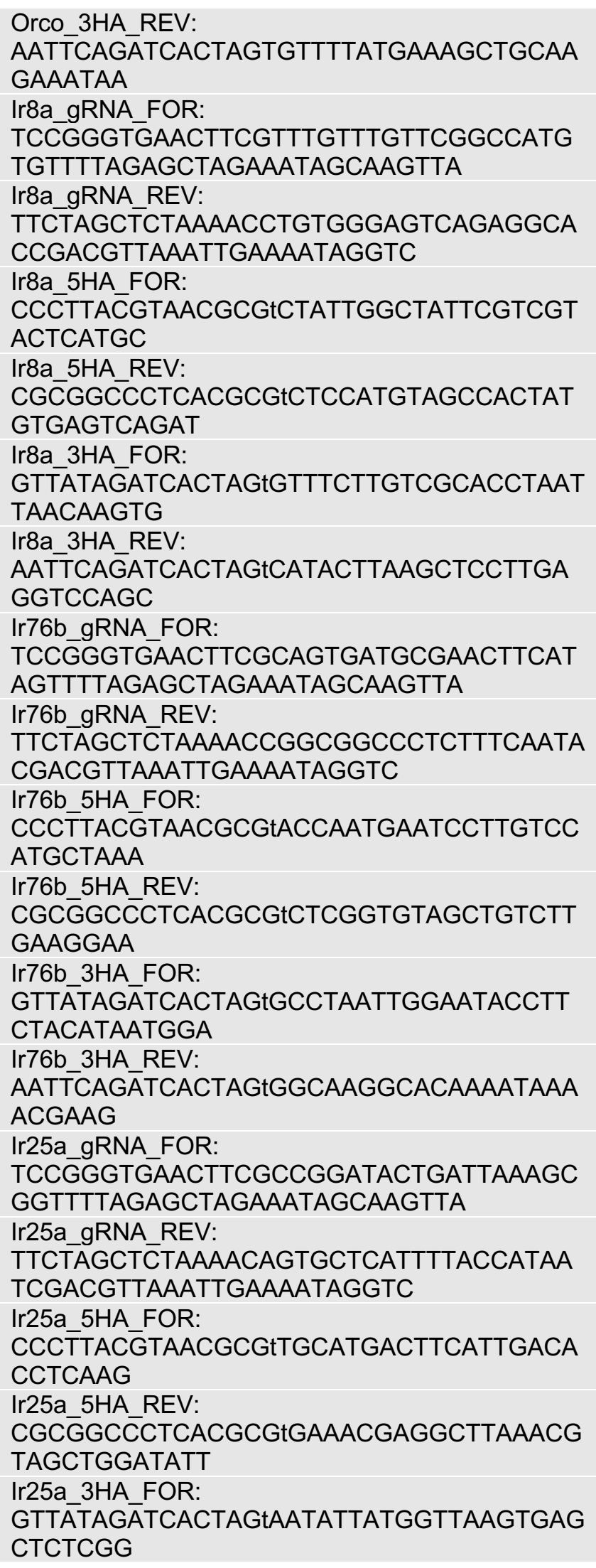

Integrated DNA

Technologies; This paper

Integrated DNA

N/A

Technologies; This paper

Integrated DNA

$\mathrm{N} / \mathrm{A}$

Technologies; This paper

Integrated DNA

N/A

Technologies; This paper

Integrated DNA

N/A

Technologies; This paper

Integrated DNA

N/A

Technologies; This paper

Integrated DNA

N/A

Technologies; This paper

Integrated DNA

N/A

Technologies; This paper

Integrated DNA

N/A

Technologies; This paper

Integrated DNA

N/A

Technologies; This paper

Integrated DNA

N/A

Technologies; This paper

Integrated DNA

N/A

Technologies; This paper

Integrated DNA

N/A

Technologies; This paper

Integrated DNA

$\mathrm{N} / \mathrm{A}$

Technologies; This paper

Integrated DNA

N/A

Technologies; This paper

Integrated DNA

N/A

Technologies; This paper

Integrated DNA

N/A

Technologies; This paper

Integrated DNA

$\mathrm{N} / \mathrm{A}$

Technologies; This paper

\section{/A}

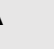




\begin{tabular}{|c|c|c|}
\hline $\begin{array}{l}\text { Ir25a_3HA_REV: } \\
\text { AATTCAGATCACTAGtCAAAGCTAAGTTCATCGT } \\
\text { CATAGAGAC }\end{array}$ & $\begin{array}{l}\text { Integrated DNA } \\
\text { Technologies; This paper }\end{array}$ & $N / A$ \\
\hline $\begin{array}{l}\text { Orco_Seq_FOR: } \\
\text { GATGTTCTGCTCTTGGCTGATATTC }\end{array}$ & $\begin{array}{l}\text { Integrated DNA } \\
\text { Technologies; This paper }\end{array}$ & $\mathrm{N} / \mathrm{A}$ \\
\hline $\begin{array}{l}\text { Ir8a_Seq_FOR: } \\
\text { CATCGACTTCATCATCAGGCTTTCG }\end{array}$ & $\begin{array}{l}\text { Integrated DNA } \\
\text { Technologies; This paper }\end{array}$ & $\mathrm{N} / \mathrm{A}$ \\
\hline $\begin{array}{l}\text { Ir76b_Seq_FOR: } \\
\text { CAACGATATCCTCACGAAGAACAAGC }\end{array}$ & $\begin{array}{l}\text { Integrated DNA } \\
\text { Technologies; This paper }\end{array}$ & $\mathrm{N} / \mathrm{A}$ \\
\hline $\begin{array}{l}\text { Ir25a_Seq_FOR: } \\
\text { CGAAAGGATACAAAGGATACTGCAT }\end{array}$ & $\begin{array}{l}\text { Integrated DNA } \\
\text { Technologies; This paper }\end{array}$ & $\mathrm{N} / \mathrm{A}$ \\
\hline $\begin{array}{l}\text { HACK_Seq_REV: } \\
\text { TGTATTCCGTCGCATTTCTCTC }\end{array}$ & $\begin{array}{l}\text { Integrated DNA } \\
\text { Technologies; This paper }\end{array}$ & $N / A$ \\
\hline $\begin{array}{l}\text { IVS-FOR: } \\
\text { TGGGTTGGACTCAGGGAATAGATCTAAAAGGTA } \\
\text { GGTTCAACCACT }\end{array}$ & $\begin{array}{l}\text { Integrated DNA } \\
\text { Technologies; This paper }\end{array}$ & $N / A$ \\
\hline $\begin{array}{l}\text { EcoRI-SV40-REV: } \\
\text { GCTTACGTCAGAATTCAGATCGATCCAGACATG } \\
\text { ATAAGA }\end{array}$ & $\begin{array}{l}\text { Integrated DNA } \\
\text { Technologies; This paper }\end{array}$ & $N / A$ \\
\hline $\begin{array}{l}\text { Tsp42Ej_FOR: } \\
\text { GAGAAGTCGTTTCCCATAACACCCT }\end{array}$ & $\begin{array}{l}\text { Integrated DNA } \\
\text { Technologies; This paper }\end{array}$ & $N / A$ \\
\hline $\begin{array}{l}\text { Tsp42Ej_REV: } \\
\text { GAGGAGCAGTTTTCGGAGTCGCCTTC }\end{array}$ & $\begin{array}{l}\text { Integrated DNA } \\
\text { Technologies; This paper }\end{array}$ & $N / A$ \\
\hline $\begin{array}{l}\text { HCR probe sets: Ir76b, Ir40a, Ir51a, Ir60a, Ir62a, } \\
\text { Ir76a, Ir93a, Orco (see Figure 6-Source Data } 2 \text { for } \\
\text { sequences) }\end{array}$ & $\begin{array}{l}\text { Molecular Instruments; } \\
\text { This paper }\end{array}$ & $\mathrm{N} / \mathrm{A}$ \\
\hline \multicolumn{3}{|l|}{ Recombinant DNA } \\
\hline$p H A C K-Q F 2$ & $\begin{array}{l}\text { Addgene; (Lin and Potter, } \\
\text { 2016a) }\end{array}$ & $\begin{array}{l}\text { Plasmid\# 80274; } \\
\text { RRID: } \\
\text { Addgene_80274 }\end{array}$ \\
\hline p10XQUAS-CsChrimson-SV40 & This paper & $\begin{array}{l}\text { Plasmid\# } 163629 \\
\text { RRID: } \\
\text { Addgene } 163629\end{array}$ \\
\hline pHACK-QF2 Orco & This paper & N/A \\
\hline pHACK-QF2Ir8a & This paper & $\mathrm{N} / \mathrm{A}$ \\
\hline$p H A C K-Q F 2^{\mid r 76 b}$ & This paper & $N / A$ \\
\hline pHACK-QF2 $2^{1 / 25 a}$ & This paper & $\mathrm{N} / \mathrm{A}$ \\
\hline \multicolumn{3}{|l|}{ Software and Algorithms } \\
\hline Fiji (ImageJ) & (Schindelin et al., 2012) & https://imagej.net/Fiji \\
\hline GraphPad Prism 8.0 & GraphPad Software & www.graphpad.com \\
\hline AutoSpike & Syntech & $\begin{array}{l}\text { http://www.ockenfels } \\
= \\
\text { syntech.com/product } \\
\text { s/signal-acquisition- } \\
\text { systems-2/ }\end{array}$ \\
\hline LabChart Pro v8 & ADInstruments & $\begin{array}{l}\text { https://www.adinstru } \\
\text { ments.com/support/d } \\
\text { ownloads/windows/l } \\
\text { abchart }\end{array}$ \\
\hline Adobe Illustrator CS6 & Adobe, Inc. & $\begin{array}{l}\text { https://www.adobe.c } \\
\text { om/products/illustrat } \\
\text { or.html }\end{array}$ \\
\hline
\end{tabular}




\begin{tabular}{|c|c|c|}
\hline Adobe Photoshop CS6 & Adobe, Inc. & $\begin{array}{l}\text { https://www.adobe.c } \\
\text { om/products/photosh } \\
\text { op.html }\end{array}$ \\
\hline MacVector 16.0 & MacVector, Inc. & $\begin{array}{l}\text { https://macvector.co } \\
\underline{\mathrm{m} /}\end{array}$ \\
\hline Zen Black & Carl Zeiss Microscopy & $\begin{array}{l}\frac{\overline{\mathrm{htt}} \mathrm{ps}: / / \text { www.zeiss.co }}{\mathrm{m} / \mathrm{microscopy} / \mathrm{us} / \mathrm{pro}} \\
\text { ducts/microscope- } \\
\text { software/zen.html }\end{array}$ \\
\hline Venn Diagram web tool & $\begin{array}{l}\text { VIB / UGent } \\
\text { Bioinformatics \& } \\
\text { Evolutionary Genomics }\end{array}$ & $\begin{array}{l}\text { http://bioinformatics. } \\
\text { psb.ugent.be/webtoo } \\
\text { Is/Venn/ }\end{array}$ \\
\hline Amira & Thermo Fisher Scientific & 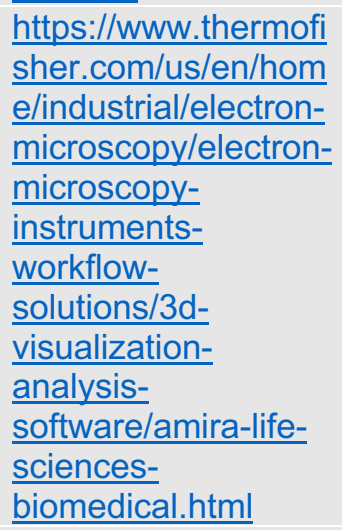 \\
\hline Python packages & (Schlegel et al., 2021) & $\begin{array}{l}\text { https://github.com/sc } \\
\text { hlegelp/navis; } \\
\text { https://github.com/fly } \\
\text { connectome/fafbseg- } \\
\text { py }\end{array}$ \\
\hline Q-Capture Pro 7 & Teledyne QImaging & $\begin{array}{l}\text { https://www.qimagin } \\
\text { g.com/home }\end{array}$ \\
\hline
\end{tabular}

1194 Resource Availability

1195 Lead Contact

1196 Further information and requests for resources and reagents should be directed to and will be

1197 fulfilled by the Lead Contact, Christopher J. Potter (cpotter@jhmi.edu).

1198 Materials Availability

1199 Fly lines generated in this study have been deposited to Bloomington Drosophila Stock Center.

1200 Data and Code Availability

1201 snRNAseq dataset analyzed in this paper is published in (McLaughlin et al., 2021). Sequencing 1202 reads and pre-processed sequencing data are available in the NCBI Gene Expression Omnibus 
1203 (GSE162121). Python code for generating figures is publicly available on GitHub

1204 (https://github.com/colleen- mclaughlin/ORN seq/). VM6 reconstructions using FlyWire can be

1205 viewed at https://flywire.ai/\#links/Task2021a/all. All other data are available upon request.

\section{Experimental Model and Subject Details}

1207 Fly Husbandry

1208 Fly stocks were maintained at $20-25^{\circ} \mathrm{C}$ on standard cornmeal-agar food. Male and female flies

1209 used for experiments were 3 - 11 days old, unless otherwise noted.

1210 Fly Stocks

1211 Fly lines used in this paper can be found in the Key Resources Table.

1212 Generation of QUAS-CsChrimson: the sequence of CsChrimson.Venus was PCR

1213 amplified from the genomic DNA of UAS-CsChrimson.mVenus flies (Klapoetke et al., 2014) and

1214 cloned into the 10XQUAS vector (Addgene \#163629). Fly line was established through random

1215 P-element insertion. Cloning was confirmed with Sanger sequencing (Genewiz) before being

1216 sent for injection (Rainbow Transgenic Flies, Inc). Primers used for PCR amplification and In-

1217 Fusion cloning:

1218 IVS-FOR:

1219 TGGGTTGGACTCAGGGAATAGATCTAAAAGGTAGGTTCAACCACT

1220 EcoRI-SV40-REV:

1221 GCTTACGTCAGAATTCAGATCGATCCAGACATGATAAGA

1222 While performing co-labeling experiments, we discovered that several OrX-Gal4 lines

1223 label multiple glomeruli, and thus do not accurately represent single OSN classes. These lines

1224 were excluded from analyses and should be used with caution: Or33c-Gal4 (BDSC\# 9966),

1225 Or42a-Gal4 (BDSC\# 9970), Or43b-Gal4 (BDSC\# 23894), Or59b-Gal4 (BDSC\# 23897), Or65a-

1226 Gal4 (BDSC\# 9994), Or85a-Gal4 (BDSC\# 23133), Or85b-Gal4 (BDSC\# 23911). We also found

1227 that the following Or35a lines label the newly identified VM6I glomerulus in addition to VC3: 
Or35a-Gal4 (BDSC\# 9967), Or35a-Gal4 (BDSC\# 9968), Or35a-mCD8.GFP (BDSC\# 52624), as

1229 well as an Or35a-Gal4 line from the Carlson lab (Yao et al., 2005).

\section{Generation of HACK Knock-in Lines}

1231 The HACK knock-in approach requires two components: a donor construct and Cas9 (Lin and

1232 Potter, 2016a). The donor includes gRNAs specific to the target gene, as well as the template

1233 for HDR-mediated insertion of T2A-QF2 into the genome (Figure 2A, middle row). This template

1234 includes $\sim 1 \mathrm{~kb}$ homology arms directly up and downstream of the gene's stop codon flanking a cassette containing T2A-QF2 and a 3XP3-mCherry fluorescent eye marker (see Figure 2-Figure Supplement 1D-E and Figure 2-Figure Supplement 3A-B). Outside of these homology arms, the construct has two RNA polymerase III U6 promoters driving independent expression of two gRNAs specific to the region around the target gene's stop codon (Port et al., 2014). Two gRNAs were used to increase the probability of successfully inducing double stranded breaks in

1240 the target (Port et al., 2014). The knock-in construct replaces the target gene's stop codon

1241 (Figure 2A, bottom row), and introduces a transcriptional stop at the end of QF2. The donor construct can be supplied in one of two ways (Figure 2-Figure Supplement 1).

1243 The first is to inject the HACK construct directly into embryos expressing Cas9 in their germline 1244 (direct injection method) (Figure 2-Figure Supplement 1A-B). The second approach is to 1245 establish transgenic donor lines through random $P$-element insertion or $\Phi C 31$ integration 1246 (Bischof et al., 2007; Gloor et al., 1991; Groth et al., 2004) of the construct into the genome, 1247 followed by genetic crosses with germline Cas9 flies for generation of the knock-in (cross 1248 method)(Figure 2-Figure Supplement 1C). Only one (direct injection method) or two (cross 1249 method) generations of crosses are required for the creation of a knock-in line (Figure 2-Figure 1250 Supplement 1B-C). The HACK 3XP3-mCherry selection marker is bright but shows positional 1251 effects (Figure 2-Figure Supplement 3A). Potential knock-in flies can be screened at the adult 1252 stage (Figure 2-Figure Supplement 3A), or at the larval or pupal stages (Figure 2-Figure

1253 Supplement 1D). We generated T2A-QF2 knock-in lines for all four co-receptor genes using the 
1254 direct injection method. Additionally, we tested the feasibility of the cross approach with two 1255 genes: Orco and Ir25a. Knock-ins were confirmed by PCR genotyping and sequencing (Figure

1256 2-Figure Supplement 3B-D), and by crosses to a QUAS-GFP reporter to check for expression in 1257 the brain (QUAS-mCD8::GFP was used only to establish the Orco-T2A-QF2 knock-in line, after 1258 which the reporter was removed via genetic crosses. For all antennal lobe analyses, we used 1259 the 10XQUAS-6XGFP reporter line). We found no difference in expression pattern in the brain 1260 between these two approaches (Figure 2-Figure Supplement 1G). After establishing a knock-in 1261 line, the 3XP3-mCherry marker can be removed via Cre recombination (Siegal and Hartl, 1996).

1262 This can be useful as 3XP3-mCherry is expressed broadly throughout the fly nervous system 1263 and can interfere with red fluorescent reporters (Figure 2-Figure Supplement 1E). We produced 1264 two unmarked knock-in lines (for Orco and Ir25a) and confirmed no difference in brain GFP expression between marked and unmarked lines (Figure 2-Figure Supplement 1F).

1267 as the number of potentially HACKed knock-in flies (mCherry+), divided by the total number of 1268 flies from the given cross ( $G_{1}$ or $F_{2}$ progeny; see Figure 2-Figure Supplement $\left.1 A-C\right)$. We further 1269 calculated the percentage of founders producing knock-in lines, as this gives an indication of 1270 effort (how many initial crosses need to be set up to produce a knock-in). The aggregate 1271 efficiency rates for a given target locus ranged from $8 \%$ for Ir8a to $33 \%$ for Orco (Table 1); 1272 however, for individual crosses, efficiency rates were as high as 100\% (see Table 1-Source 1273 Data 1), meaning that all progeny were potential mCherry+ knock-ins. For the two genes for 1274 which we created knock-in lines via both direct injection and genetic cross (Orco and Ir25a), we 1275 found efficiency rates comparable between approaches (Orco: 33\% for direct injection, 28\% for 1276 cross; Ir25a: $23 \%$ for direct injection, $24 \%$ for cross). For the direct injection approach, we 1277 tested 51 independent knock-in lines across the four target genes and found $100 \%$ to be 1278 correctly targeted events (Table 1). However, for the genetic cross approach, of the 32 
1279 independent knock-in lines tested for the two target genes, 6 ( 19\%) had the HACK mCherry 1280 eye marker but did not have QF2-driven GFP expression in the brain.

1281 Information on plasmid construction can be found in Method Details below. All

1282 Drosophila embryo injections were performed by Rainbow Transgenic Flies, Inc. (Camarillo,

1283 CA). For HACKing via genetic cross, Orco-T2A-QF2 and Ir25a-T2A-QF2 constructs were

1284 injected into $w^{1118}$ flies for $P$-element insertion, and donor lines were established on the second

1285 or third chromosomes by crossing to double balancers (see Key Resources Table). Donor lines

1286 were then crossed to Vas-Cas9 (BDSC\# 51323). Knock-in lines were established from cis-

1287 chromosomal HACK (donor line on same chromosome as target gene) (Lin and Potter, 2016a).

1288 For HACKing via direct injection, knock-in constructs were injected into the following lines: Vas-

1289 Cas9 (BDSC\# 51324) for Ir8a; Act5C-Cas9 (BDSC\# 54590) for Orco, Ir76b, and Ir25a. The

1290 following lines were used to verify knock-in expression: QUAS-mCD8::GFP (BDSC\# 30003),

1291 10XQUAS-6XGFP (BDSC\# 52264). Knock-in lines were confirmed by PCR genotyping

1292 (Phusion, NEB) and Sanger sequencing (Genewiz). Unmarked Orco-T2A-QF2 and Ir25a-T2A-

1293 QF2 knock-in lines were generated by crossing mCherry+ knock-in flies to the Crey+1B fly line

1294 (see Key Resources Table) (Siegal and Hartl, 1996).

1295

To investigate the effect of T2A-QF2 knock-in on gene function, we performed single

1296 sensillum recordings (SSR) on homozygous flies for each co-receptor knock-in (Figure 2-Figure

1297 Supplement 2), comparing their responses to wildtype flies to panels of Orco- and Ir-dependent

1298 odorants (Abuin et al., 2011; de Bruyne et al., 2001; Lin and Potter, 2015). In ab2 basiconic

1299 sensilla, Orco-T2A-QF2 knock-in flies had slightly lower baseline activity as compared to

1300 wildtype (Figure 2-Figure Supplement 2A); however, there were no significant differences in

1301 odor-evoked activity between these two genotypes across all stimuli tested (Figure 2-Figure

1302 Supplement 2B). In ac2 coeloconic sensilla, responses of Ir8a-T2A-QF2 knock-in flies to

1303 hexanol and cadaverine were slightly lower than wildtype (Figure 2-Figure Supplement 2D);

1304 however, these are not typically considered Ir8a-dependent odorants. Responses of the Ir8a- 
T2A-QF2 knock-in to Ir8a-dependent odorants (Abuin et al., 2011) were similar to wildtype

1306

1307

1308

1309

1310

1311

1312

1313

1314

1315

1316

1317

1318

1319

1320

1321

1322

1323

1324

1325

1326

1327

1328

1329 controls (example trace in Figure 2-Figure Supplement 2C, quantification in Figure 2-Figure Supplement 2D). Responses of Ir76b-T2A-QF2 knock-in ac2 neurons to phenethylamine and acetic acid differed slightly from wildtype controls (Figure 2-Figure Supplement 2E-F). The reasons for this are unclear. The largest difference in responses between a knock-in and wildtype were for Ir25a-T2A-QF2 (Figure 2-Figure Supplement 2G-H); the knock-in has significantly reduced or abolished responses to Ir25a-dependent odorants, recapitulating an Ir25a mutant phenotype (Abuin et al., 2011; Silbering et al., 2011). See also Figure 2-Source

\section{Data 1.}

\section{Method Details}

\section{Plasmid Construction}

The construction of $Q F 2^{X-H A C K}$ knock-in plasmids requires three steps of cloning, as previously described (Lin and Potter, 2016a). All knock-in constructs were created using the pHACK-QF2 plasmid (Addgene \#80274) as the backbone. The backbone was digested with the following enzymes for cloning: Mlul for the 5' homology arms; Spel for the 3' homology arms; and Bbs/ for the gRNAs. All cloning was performed using In-Fusion Cloning (Clontech \#639645). The homology arms were PCR amplified from genomic DNA extracted from wildtype flies using the DNeasy Blood and Tissue Kit (Qiagen \#69506), while the gRNAs were PCR amplified using the pHACK-QF2 backbone as a template, with the primers themselves containing the gRNA target sequences. All homology arms were approximately 1 kb (Orco: 5HA = 1012 bp, 3HA = 1027 bp; Ir8a: 5HA = 1027 bp, 3HA = 1079 bp; Ir76b: 5HA = 997 bp, 3HA = 956 bp; Ir25a: 5HA = 1119 bp, 3HA = 990 bp). gRNAs were selected by analyzing the region around the stop codon of each gene using an online tool (https://flycrispr.org/) (Gratz et al., 2014). When possible, gRNAs were chosen to minimize potential off-target cleavage sites (zero predicted for Orco, Ir8a, and Ir76b; one predicted for Ir25a, discussed below). They were selected such that: one gRNA 
1330 targeted upstream of the stop codon, within the last exon of the gene; the second gRNA

1331 targeted downstream of the stop codon, within the 3'UTR; and the two gRNAs were $<100$ bp

1332 apart. In order to prevent the gRNAs from targeting the homology arms, three synonymous

1333 nucleotide substitutions were made in each homology arm. The final knock-in lines did not

1334 always have all three substitutions (see Figure 2-Figure Supplement 3D), possibly due to PCR

1335 or HDR error. Note that due to the way the primers are designed, each targeted gene loses a

1336 small portion of its native 3'UTR (Orco = $72 \mathrm{bp}, \operatorname{Ir} 8 \mathrm{a}=31 \mathrm{bp}, \operatorname{Ir} 76 \mathrm{~b}=27 \mathrm{bp}, \operatorname{Ir} 25 \mathrm{a}=24 \mathrm{bp}$ ).

1337 Cloning was confirmed with Sanger sequencing (Genewiz) before being sent for injection

1338 (Rainbow Transgenic Flies, Inc). Below are the gRNAs used for each gene, with the PAM

1339 sequence in parentheses.

1340

1341 Orco:

1342 CTGCACCAGCACCATAAAGT (AGG)

1343 GCACAGTGCGGAGGGGGCAA (GGG)

1344

1345 Ir8a:

1346 GTTTGTTTGTTCGGCCATGT (TGG)

1347 GGTGCCTCTGACTCCCACAG (TGG)

1348

1349 Ir76b:

1350 GCAGTGATGCGAACTTCATA (TGG)

1351 GTATTGAAAGAGGGCCGCCG (AGG)

1352

1353 Ir25a:

1354 GCCGGATACTGATTAAAGCG (CGG)

1355 ATTATGGTAAAATGAGCACT (CGG) 
1357 PRIMERS

1358 Italics $=$ In-Fusion cloning 15 b.p. overhang; bold $=$ gRNA; lowercase $=$ adding back restriction

1359 site; $\underline{\text { underline }}=$ synonymous substitution to prevent Cas9 targeting of donor construct.

1361 PCR Primers for Cloning:

1362 Orco_gRNA_FOR:

1363 TCCGGGTGAACTTCGCACAGTGCGGAGGGGGCAAGTTTTAGAGCTAGAAATAGCAAGTTA

1364 Orco_gRNA_REV:

1365 TTCTAGCTCTAAAACACTTTATGGTGCTGGTGCAGCGACGTTAAATTGAAAATAGGTC

1366 Orco_5HA_FOR:

1367 CCCTTACGTAACGCGICAGCTTGTTTGACTTACTTGATTAC

1368 OrCo_5HA_REV:

1369 CGCGGCCCTCACGCGtCTTGAGCTGIACAAGIACCATAAAGT

1370 Orco_3HA_FOR:

1371 GTTATAGATCACTAGtCTCÁGIACTㅁTGCAACCAGCAATA

1372 OrCo_3HA_REV:

1373 AATTCAGATCACTAGtGTTTTATGAAAGCTGCAAGAAATAA

1374

1375 Ir8a_gRNA_FOR:

1376 TCCGGGTGAACTTCGTTTGTTTGTTCGGCCATGTGTTTTAGAGCTAGAAATAGCAAGTTA

1377 Ir8a_gRNA_REV:

1378 TTCTAGCTCTAAAACCTGTGGGAGTCAGAGGCACCGACGTTAAATTGAAAATAGGTC

1379 Ir8a_5HA_FOR:

1380 CCCTTACGTAACGCGtCTATTGGCTATTCGTCGTACTCATGC

1381 Ir8a_5HA_REV: 
1383 Ir8a_3HA_FOR:

1384 GTTATAGATCACTAGtGTTTCTTGTCGCACCTAATTAACAAGTG

1385 Ir8a_3HA_REV:

1386 AATTCAGATCACTAGtCATACTTAAGCTCCTTGAGGTCCAGC

1388 Ir76b_gRNA_FOR:

1389 TCCGGGTGAACTTCGCAGTGATGCGAACTTCATAGTTTTAGAGCTAGAAATAGCAAGTTA 1390 Ir76b_gRNA_REV:

1391 TTCTAGCTCTAAAACCGGCGGCCCTCTTTCAATACGACGTTAAATTGAAAATAGGTC 1392 Ir76b_5HA_FOR:

1393 CCCTTACGTAACGCGIACCAATGAATCCTTGTCCATGCTAAA

1394 Ir76b_5HA_REV:

1395 CGCGGCCCTCACGCGtCTCGGTGTAGCTGTㅡTTGAAGGA프

1396 Ir76b_3HA_FOR:

1397 GTTATAGATCACTAGtGCCTAATTGGAATACCTTCTACATAATGGA

1398 Ir76b_3HA_REV:

1399 AATTCAGATCACTAGtGGCAAGGCACAAAATAAAACGAAG

1400

1401 Ir25a_gRNA_FOR:

1402 TCCGGGTGAACTTCGCCGgATACTGATTAAAGCGGTTTTAGAGCTAGAAATAGCAAGTTA 1403 Ir25a_gRNA_REV:

1404 TTCTAGCTCTAAAACAGTGCTCATTTTACCATAATCGACGTTAAATTGAAAATAGGTC 1405 Ir25a_5HA_FOR:

1406 CCCTTACGTAACGCGtTGCATGACTTCATTGACACCTCAAG

1407 Ir25a_5HA_REV: 
1409 Ir25a_3HA_FOR:

1410 GTTATAGATCACTAGtAATATTATGGTIAAGTGAGCICTCGG

1411 Ir25a_3HA_REV:

1412 AATTCAGATCACTAGtCAAAGCTAAGTTCATCGTCATAGAGAC

1414 Genotyping and Sequencing Primers (PCR fragment size):

1415 Orco_Seq_FOR ( 2kb):

1416 GATGTTCTGCTCTTGGCTGATATTC

1417 Ir8a_Seq_FOR ( 1.9kb):

1418 CATCGACTTCATCATCAGGCTTTCG

1419 Ir76b_Seq_FOR ( 1.9kb):

1420 CAACGATATCCTCACGAAGAACAAGC

1421 Ir25a_Seq_FOR ( 1.9kb):

1422 CGAAAGGATACAAAGGATACTGCAT

1423 HACK_Seq_REV (same for all):

1424 TGTATTCCGTCGCATTTCTCTC

1425 Checking for Off-target Effects

1426 One of the gRNAs for QF2 Ir25a-HACK had one predicted potential off-target cut site in the genome,

1427 in the tetraspanin 42ej (Tsp42Ej) gene. We sequenced this locus in the Ir25a-T2A-QF2 knock-in

1428 line and compared the sequence to our wildtype lab stock. We found no evidence of indels in

1429 the knock-in line. Primers used:

1430 Tsp42Ej_FOR:

1431 GAGAAGTCGTTTCCCATAACACCCT

1432 Tsp42Ej_REV:

1433 GAGGAGCAGTTTTCGGAGTCGCCTTC 


\section{HACK Marker Screening}

1435 Adult flies were anaesthetized on $\mathrm{a} \mathrm{CO}_{2}$ pad and screened in one of two ways: either with a

1436 Nightsea Stereo Microscope Fluorescence Adapter with the green SFA-GR LED light source

1437 (Nightsea LLC, Lexington, MA) and viewed with a Zeiss Stemi SV6 stereo microscope; or

1438 illuminated with an X-Cite $120 \mathrm{Q}$ excitation light source and viewed with a Zeiss SteREO

1439 Discovery V8 microscope equipped with a ds-Red filter.

Whole Animal Imaging

1441 Whole adults were anaesthetized on ice before imaging. Whole larvae, pupae, or freshly

1442 dissected adult heads were affixed to slides with clear nail polish before imaging. All animals

1443 were imaged on an Olympus SZX7 microscope equipped with GFP and RFP filters. Animals

1444 were illuminated with an X-Cite Series 120Q light source. Images were acquired using a

1445 QImaging QIClick Cooled digital CCD camera and Q-Capture Pro 7 software. Multiple images

1446 were taken at different focal planes and then merged in Photoshop (CS6). Gain was adjusted in

1447 Fiji. Images appear in the following figures/panels: Figure 2B, D, F, H; Figure 2-Figure

1448 Supplement 1D; Figure 2-Figure Supplement 3A; Figure 2-Figure Supplement 4.

1449 Immunohistochemistry

1450 All flies were used at 3- to 11-days old. Apart from the cryosection protocols and portions of the

1451 antennal whole-mount protocol, all immunostaining steps were done on a nutator. All steps

1452 involving or following the addition of fluorescently conjugated secondary antibodies were done

1453 in the dark.

$1454 \quad$ Brain and ventral nerve cord (VNC) staining was performed as in (Xie et al., 2018).

1455 Tissue was dissected in PBS and then fixed for 20 minutes at room temperature (RT) in 4\%

1456 paraformaldehyde in PBT (1XPBS with 0.3\% Triton X-100). After fixation, tissue was quickly

1457 rinsed three times with PBT, then put through three longer washes in PBT at RT (at least 15

1458 minutes each). Tissue was blocked in PBT $+5 \%$ normal goat serum (NGS) at RT for at least 30 
1459 minutes, then transferred to block + primary antibody solution, and incubated at $4^{\circ} \mathrm{C}$ in primary

1460 antibodies for 1-2 days. Tissue was then washed three times with PBT at RT (at least 15

1461 minutes per wash) and incubated in secondary antibody solution in block at $4^{\circ} \mathrm{C}$ for 1 day.

1462 Tissue was washed three final times with PBT at RT for 15 minutes each, and then mounted in

1463 SlowFade gold (Thermo Fisher Scientific \#S36936). For experiments in which the 10XQUAS-

1464 6XGFP or 20XUAS-6XGFP reporters were used, the endogenous, unstained GFP signal was

1465 visualized, and no secondary green antibodies were used. Primary antibodies used: mouse anti-

1466 nc82 (DSHB, 1:25), rat anti-mCD8 (Thermo Fisher \#14-0081-82, 1:100 or 1:200). Secondary

1467 antibodies used: Cy3 goat anti-rat (Jackson ImmunoResearch \#112-165-167, 1:200), Cy3 goat

1468 anti-mouse (Jackson ImmunoResearch \#115-165-166, 1:200), Alexa 647 goat anti-rat (Jackson

1469 ImmunoResearch \#112-605-167, 1:200), Alexa 647 goat anti-mouse (Jackson

1470 ImmunoResearch \#115-605-166, 1:200).

Whole-mount staining of maxillary palps was performed according to the brain staining

1472 protocol above, with the exception of a shorter fixation step (15 minutes). Primary antibodies

1473 used: rabbit anti-Ir25a (gift from Richard Benton, University of Lausanne, 1:100), rabbit anti-

1474 Orco (gift from Leslie Vosshall, Rockefeller University, 1:100), rat anti-elav (DSHB, 1:100).

1475 Secondary antibodies used: Cy3 goat anti-rabbit (Jackson ImmunoResearch \#111-165-144,

1476 1:200), Alexa 647 goat anti-rabbit (Jackson ImmunoResearch \#111-605-144, 1:200), Alexa 647

1477 goat anti-rat (Jackson ImmunoResearch \#112-605-167, 1:200). For whole-mount staining of

$1478 \mathrm{Orco}^{2}$ mutant palps, 3-day-old flies were used to check for Orco expression before neurons

1479 degenerate (Task and Potter, 2021).

1480 The protocol for whole-mount staining of antennae was adapted from (Karim et al., 2014;

1481 Saina and Benton, 2013; Younger et al., 2020). Fly heads were dissected into CCD buffer (50

1482 units chitinase, 1000 units chymotrypsin (25 mg of 40 units/mg), $10 \mathrm{~mL}$ HEPES larval buffer

1483 (119 mM NaCl, 48 mM KCl, 2 mM CaCl 2,2 mM MgCl 2,25 mM HEPES), $100 \mu$ l DMSO) on ice,

1484 then warmed on a $37^{\circ} \mathrm{C}$ heat block for $10 \mathrm{~min}$. Heads were incubated in CCD buffer at $37^{\circ} \mathrm{C}$ 
while rotating for 1 hour 20 minutes. Antennae were subsequently dissected off heads into

1486 fixative solution (4\% PFA in PBT). All subsequent steps were done without rotation to prevent

1487 antennae from sticking to the walls or lids of the tubes. Antennae were fixed at room

1488 temperature for $40 \mathrm{~min}$, then washed with PBT three times at room temperature, at least 15

1489 minutes each time, and blocked in PBT plus 5\% NGS for at least 1 hour at room temperature.

1490 Antennae were incubated in primary antibodies in blocking solution at $4^{\circ} \mathrm{C}$ for four days, washed

1491 three times for 15 minutes each at room temperature, and incubated in secondary antibody

1492 solution at $4^{\circ} \mathrm{C}$ for three days. Antennae were then washed three times for 15 minutes each

1493 time at room temperature and mounted in SlowFade Gold. Primary antibody: rabbit anti-Orco

1494 (gift from Leslie Vosshall, 1:100); secondary antibody: Cy3 goat anti-rabbit (Jackson

1495 ImmunoResearch \#111-165-144, 1:200). The endogenous GFP signal was visualized.

1497 dissected and lined up in cryomolds (Tissue-Tek \#4565), covered with OCT compound (Tissue-

1498 Tek \#4583), and frozen at $-80^{\circ} \mathrm{C}$. Samples were sectioned at $\sim 12 \mu \mathrm{m}$ on a Microm HM 500

1499 cryostat (Microm International GmbH, Walldorf, Germany) and collected on SuperFrost Plus

1500 slides (Fisher \#12-550-15). Slides were stored at $-80^{\circ} \mathrm{C}$ until further processing. Slides were

1501 fixed at RT for 15 minutes in 4\% paraformaldehyde in PBT (1XPBS with 0.3\% Triton X-100),

1502 washed three times in PBT at RT (15 minutes each), blocked at RT for at least 30 minutes in

1503 PBT $+2.5 \%$ NGS $+2.5 \%$ normal donkey serum (NDS), then incubated overnight at $4^{\circ} \mathrm{C}$ in

1504 primary antibodies in fresh block solution in a special humidified chamber. On the next day,

1505 slides were washed three times (15 minutes each) with PBT at RT, and then incubated in

1506 secondary antibodies in block at $4^{\circ} \mathrm{C}$ overnight in the same humidified chamber covered in foil.

1507 Finally, slides were washed three times (15 minutes each) with PBT at RT. DAPI $(1: 10,000)$ was

1508 included in the first wash as a nuclear counterstain. After washes, slides were mounted in

1509 SlowFade gold (Thermo Fisher Scientific \#S36936). Primary antibody used: guinea pig anti-Ir8a 
1510 (gift from Richard Benton, University of Lausanne, 1:1,000). Secondary antibody used: Cy3 1511 donkey anti-guinea pig (Jackson ImmunoResearch \#706-165-148, 1:200).

1512 For sacculus staining, 7-10 day old flies were placed in an alignment collar. Their heads 1513 were encased in OCT (Tissue-Plus Fisher) in a silicone mold, frozen on dry ice, and snapped 1514 off. The head blocks were stored in centrifuge tubes at $-80^{\circ} \mathrm{C}$. A Leica cryostat was used to 1515 collect $20 \mu \mathrm{m}$ sections of antennae. Immunohistochemical staining was carried out by fixing 1516 tissue in $4 \%$ paraformaldehyde for 10 minutes, followed by three 5 minute washes in 1 XPBS.

1517 Tissue was washed in 1XPBS containing $0.2 \%$ Triton-X (PBST) for 30 minutes to permeabilize 1518 the cuticle. Lastly, tissue was washed in PBST containing 1\% Bovine Serum Albumin (BSA) to 1519 block non-specific antibody binding. Primary antibody solution was made in PBST+1\%BSA, 200 $1520 \mu \mathrm{l}$ was pipetted onto each slide under bridged coverslips, and slides were placed at $4^{\circ} \mathrm{C}$ 1521 overnight to incubate. The following day, primary antibody was removed, and slides were 1522 washed three times for 10 minutes each in PBST. Secondary antibody solution was made in 1523 PBST+1\%BSA, 200uL was pipetted onto each slide under bridged coverslips, and left at room 1524 temperature in a dark box to incubate for 2 hours. After the 2 hour incubation, slides were 1525 washed three times for 5 minutes each in PBST. After the last wash, the slides were allowed to 1526 dry in the dark staining box for $\sim 30$ minutes before being mounted in Vectashield, coverslipped, 1527 and stored at $4^{\circ} \mathrm{C}$. Primary antibodies: rabbit anti-Ir25a (gift from Richard Benton, University of 1528 Lausanne, 1:100), guinea pig anti-Ir8a (gift from Richard Benton, University of Lausanne, 1529 1:100), rabbit anti-Ir64a (gift from Greg Suh, NYU/KAIST, 1:100). Secondary antibodies: 1530 Jackson Immuno Cy3 conjugated AffiniPure 568 goat anti-rabbit (111-165-144), 1:500;

1531 AlexaFluor 568 goat anti-guinea pig (A11075), 1:500.

1532 In situ HCR

1533 Cryosectioning for antennal in situs was performed as described above. The HCR protocol was 1534 adapted from Molecular Instruments HCR v3.0 protocol for fresh frozen or fixed frozen tissue 1535 sections (Choi et al., 2018). Slides were fixed in ice-cold 4\% PFA in PBT for 15 minutes at $4^{\circ} \mathrm{C}$, 
dehydrated in an ethanol series $(50 \% \mathrm{EtOH}, 70 \% \mathrm{EtOH}, 100 \% \mathrm{EtOH}, 100 \% \mathrm{EtOH}, 5$ minutes

1537 each step), and air dried for 5 minutes at RT. Slides were then incubated in proteinase K

1538 solution in a humidified chamber for 10 minutes at $37^{\circ} \mathrm{C}$, rinsed twice with PBS and dried, then

1539 pre-hybridized for 10 minutes at $37^{\circ} \mathrm{C}$ in a humidified chamber. Slides were incubated in probe

1540 solution $\left(0.4 \mathrm{pmol} \operatorname{Ir} 76 \mathrm{~b}\right.$ probe) overnight in the $37^{\circ} \mathrm{C}$ humidified chamber. On day two, slides

1541 were washed with a probe wash buffer/SSCT series (75\% buffer/25\% SSCT, 50\% buffer/50\%

1542 SSCT, $25 \%$ buffer $/ 75 \%$ SSCT, $100 \%$ SSCT, 15 minutes each) at $37^{\circ} \mathrm{C}$, then washed for 5 min

1543 at RT with SSCT and dried. Slides were pre-amplified for $30 \mathrm{~min}$ at RT in the humidified

1544 chamber while hairpins were snap cooled (6 pmol concentration). Slides were incubated in fresh

1545 amplification buffer with hairpins overnight in a dark humidified chamber at RT. On day three,

1546 slides were rinsed in SSCT at RT $(2 \times 30$ minutes, $1 \times 10$ minutes with 1:10,000 DAPI, $1 \times 5$

1547 minutes) and mounted in SlowFade Diamond (Thermo Fisher S36972). For the overnight steps,

1548 slides were covered with HybriSlips (Electron Microscopy Sciences 70329-62) to prevent

1549 solution evaporation.

1550

The whole-mount palp in situ protocol was adapted from a combination of (Prieto-Godino

1551 et al., 2020; Saina and Benton, 2013; Younger et al., 2020) and the Drosophila whole-mount

1552 embryo protocol from Molecular Instruments (Choi et al., 2016). All steps after dissection were

1553 performed while rotating unless otherwise noted. Fly mouthparts (palps and proboscises) were

1554 dissected into CCD buffer (same as for whole-mount IHC on antennae above), incubated for 20

1555 minutes in $\mathrm{CCD}$ at $37^{\circ} \mathrm{C}$ (5 minutes on heat block, 15 minutes rotating), then pre-fixed in $4 \%$

1556 PFA in PBT for 20 minutes at RT. Tissue was washed with $0.1 \%$ PBS-Tween on ice $(4 \times 5$

1557 minutes), incubated for 1 hour at RT in $80 \%$ methanol/20\% DMSO, and washed for 10 minutes

1558 in PBS-Tween at RT. Tissue was then incubated in Proteinase K solution $(1: 1,000)$ in PBS-

1559 Tween at RT for 30 minutes, then washed in PBS-Tween at RT ( 2 × 10 minutes) and post-fixed

1560 in 4\% PFA in PBS-Tween at RT for 20 minutes. After post-fixation, tissue was washed in PBS-

1561 Tween at RT ( $3 \times 15$ minutes), then pre-hybridized in pre-heated probe hybridization buffer at 
$156237^{\circ} \mathrm{C}$ for 30 minutes. Tissue was incubated in probe solution ( $2-4$ pmol in hybridization buffer $)$

1563 at $37^{\circ} \mathrm{C}$ for two nights. On day three, tissue was washed in pre-heated probe wash buffer at

$156437^{\circ} \mathrm{C}(5 \times 10$ minutes), then washed in SSCT (1XSSC plus $1 \%$ Tween) at RT ( $2 \times 5$ minutes).

1565 Tissue was pre-amplified with RT-equilibrated amplification buffer at RT for 10 minutes, then

1566 incubated in hairpin mixture (6 pmol snap-cooled hairpins in amplification buffer) in the dark at

1567 RT overnight. On day four, tissue was washed at RT with SSCT $(2 \times 5$ minutes, $2 \times 30$ minutes,

$15681 \times 5$ minutes), then mounted in SlowFade Diamond (Thermo Fisher S36972). Sequences for all

1569 in situ probes can be found in Figure 6-Source Data 2. Example images for palp in situs can be

1570 found in Figure 6-Figure Supplement 2.

\section{Confocal Imaging and Analysis}

1572 Brains, ventral nerve cords, maxillary palps, and antennal cryosections were imaged on a Zeiss

1573 LSM 700 confocal microscope equipped with Fluar 10x/0.50 air M27, LCI Plan-Neofluar 25x/0.8

1574 water Korr DIC M27, and C-Apochromat 63x/1.2 water Korr M27 objectives. Images were

1575 acquired at $512 \times 512$-pixel resolution with $0.58,2.37$ or $6.54 \mu \mathrm{m}$ z-steps for each objective,

1576 respectively. For illustration purposes, confocal images were processed in Fiji/lmageJ to

1577 collapse Z-stacks into a single image using maximum intensity projection. Where noted, single

1578 slices or partial z projections were used as opposed to full stacks. For co-labeling experiments,

1579 Fiji was used to convert red LUTs to orange for a colorblind-friendly palette. Similarly, in Figure

1580 2-Figure Supplement 1E, Fiji was used to convert magenta LUT to blue for clarity. For Figure

1581 4B, Fiji was used to convert the two-channel maximum intensity projection to a grey LUT, and

1582 the cell-counting plug-in was used in separate channels to identify single- and double-labeled

1583 cells. Fiji was also used to adjust the gain in separate channels in all figures/images; no other

1584 image processing was performed on the confocal data. For Figure 7A, glomeruli were assigned

1585 to the categories strong, intermediate, and weak by visual inspection of the strength of their

1586 innervation compared to the previously reported glomeruli for each respective knock-in line. 
1587 Strong glomeruli generally have similar brightness/intensity of GFP signal as most of the 1588 originally reported glomeruli for the given knock-in line. For sacculus staining (Figure 5C-E), slides were imaged on a Nikon A1R confocal

1590 microscope in the UConn Advanced Light Microscopy Facility with a 40X oil immersion objective 1591 at $1024 \times 1024-$ pixel resolution. Stacks of images $(0.5 \mu \mathrm{m}$ z-step size $)$ were gathered and 1592 analyzed with ImageJ/Fiji software. Image processing was performed as described above.

1593 Magnification used:

1594 10X: Figure 1D (Brain), Figure 2-Figure Supplement 1E

1595 25X: Figure 2-Figure Supplement 1F-G, Figure 3-Figure Supplement 1A-B

1596 40X: Figure 5C-E

1597 63X: Figure 2C, E, G, I; Figure 3A-D, Figure 4B-C, Figure 5A-B, Figure 2-Figure Supplement

1598 3E-H, Figure 3-Figure Supplement 2A-E, Figure 5-Figure Supplement 1, Figure 6-Figure

1599 Supplement 2

1600 Basiconic Single Sensillum Recordings

1601 Flies were immobilized and visualized as previously described (Lin and Potter, 2015). Basiconic 1602 sensilla were identified either using fluorescent-guided SSR (for ab3 sensilla) or using reference 1603 odorants (for ab2 and pb1-3 sensilla) (de Bruyne et al., 1999; Lin and Potter, 2015). The glass 1604 recording electrode was filled with Beadle-Ephrussi Ringer's solution $(7.5 \mathrm{~g}$ of $\mathrm{NaCl}+0.35 \mathrm{~g}$ of $1605 \mathrm{KCl}+0.279 \mathrm{~g}$ of $\mathrm{CaCl}_{2}-2 \mathrm{H}_{2} \mathrm{O}$ in $1 \mathrm{~L} \mathrm{H}_{2} \mathrm{O}$ ). Extracellular activity was recorded by inserting the 1606 glass electrode into the shaft or base of the sensillum of 3- to 10-day-old flies (unless otherwise 1607 specified in the young $\operatorname{Orco}^{2}$ mutant experiments). A tungsten reference electrode was inserted 1608 into the fly eye. Signals were amplified 100X (USB-IDAC System; Syntech, Hilversum, the 1609 Netherlands), input into a computer via a 16-bit analog-digital converter and analyzed off-line 1610 with AUTOSPIKE software (USB-IDAC System; Syntech). The low cutoff filter setting was 50 $1611 \mathrm{~Hz}$, and the high cutoff was $5 \mathrm{kHz}$. Stimuli consisted of $1000 \mathrm{~ms}$ air pulses passed over odorant 1612 sources. The $\Delta$ spikes/second was calculated by counting the spikes in a $1000 \mathrm{~ms}$ window from 
$1613 \sim 500 \mathrm{~ms}$ after odorant stimuli were triggered, subtracting the spikes in a $1000 \mathrm{~ms}$ window prior

1614 to each stimulation. For ab3 recordings from wildtype, Orco ${ }^{2}$ mutant, and $\operatorname{Ir} 25 \mathrm{a}^{2}$ mutant flies,

1615 spikes were counted in a $500 \mathrm{~ms}$ window from the start of the response and multiplied by 2.

1616 Then, the spikes in the $1000 \mathrm{~ms}$ window prior to stimulation was subtracted from this to

1617 calculate the $\Delta$ spikes/second. Stimuli used: mineral oil (Sigma CAS \#8042-47-5), ethyl acetate

1618 (Sigma CAS \#141-78-6), pentyl acetate (Sigma CAS \#628-63-7), benzaldehyde (Sigma CAS

1619 \#100-52-7), ethyl butyrate (Sigma CAS \#105-54-4), hexanol (Sigma CAS \#111-27-3), e2-

1620 hexenal (Sigma CAS \#6728-26-3), geranyl acetate (Sigma CAS \# 105-87-3), 2-heptanone

1621 (Sigma CAS \# 110-43-0), 1-octen-3-ol (Sigma CAS \#3391-86-4), 2,3-butanedione (Sigma CAS

1622 \#431-03-8), phenylacetaldehyde (Sigma CAS \# 122-78-1), phenethylamine (Sigma CAS \# 64-

1623 04-0), propionic acid (Sigma CAS \#79-09-4), 1,4-diaminobutane (Sigma CAS \#110-60-1),

1624 pyrrolidine (Sigma CAS \#123-75-1), p-cresol (Sigma CAS \#106-44-5), and methyl salicylate

1625 (Sigma CAS \#119-36-8). Odorants were dissolved in mineral oil at a concentration of 1\%, and

$162620 \mu \mathrm{l}$ of solution was pipetted onto filter paper in a glass Pasteur pipette. Stimuli were delivered

1627 by placing the tip of the Pasteur pipette through a hole in a plastic pipette (Denville Scientific

$1628 \mathrm{Inc}, 10 \mathrm{ml}$ pipette) that carried a purified continuous air stream $(8.3 \mathrm{ml} / \mathrm{s})$ directed at the antenna

1629 or maxillary palp. A solenoid valve (Syntech) diverted delivery of a $1000 \mathrm{~ms}$ pulse of charcoal-

1630 filtered air $(5 \mathrm{ml} / \mathrm{s})$ to the Pasteur pipette containing the odorant dissolved on filter paper. Fresh

1631 odorant pipettes were used for no more than 5 odorant presentations. $\operatorname{Ir} 25 \mathrm{a}^{2}$ and Orco $^{2}$ mutant

1632 fly lines were outcrossed into the $w^{1118}$ wildtype genetic background. Full genotypes for ab3

1633 fgSSR were: Pin/CyO;Or22a-Gal4,15XUAS-IVS-mcd8GFP/TM6B (wildtype), Ir25a²;Or22a-

1634 Gal4,15XUAS-IVS-mcd8GFP/TM6B (Ir25a² mutant), Or22a-Gal4/10XUAS-IVS-mcd8GFP

1635 (attp40); $\operatorname{Orco}^{2}\left(\operatorname{Orco}^{2}\right.$ mutant). These stocks were made from the following Bloomington Stocks

1636 (outcrossed to the Potter lab $w^{1118}$ genetic background): BDSC\# 9951, 9952, 23130, 32186,

$163732193,41737$. 


\section{Coeloconic Single Sensillum Recordings}

Coeloconic SSR was performed similarly as for basiconic sensilla. 3-5-day old female flies were wedged in the tip of a $200 \mu$ l pipette, with the antennae and half the head exposed. A tapered glass electrode was used to stabilize the antenna against a coverslip. A BX51WI microscope (Olympus) was used to visualize the prep, which was kept under a 2,000 $\mathrm{mL} / \mathrm{min}$ humidified and purified air stream. A borosilicate glass electrode was filled with sensillum recording solution (Kaissling and Thorson, 1980) and inserted into the eye as a reference electrode. An aluminosilicate glass electrode was filled with the same recording solution and inserted into individual sensilla. Different classes of coeloconic sensilla were identified by their known location on the antenna and confirmed with their responses to a small panel of diagnostic odors. No more than 4 sensilla per fly were recorded. Each sensillum was tested with multiple odorants, with a rest time of at least 10 s between applications. The odorants used were acetic acid (Fisher, 1\%, CAS\# 64-19-7), ammonium hydroxide (Fisher, 0.1\%, CAS\# 7664-41-7), cadaverine (Sigma-Aldrich, 1\%, CAS\# 462-94-2), hexanol (ACROS Organics, 0.001\%, CAS\# 111-27-3), 2,3-butanedione (ACROS Organics, 1\%, CAS\# 431-03-8), phenethylamine (ACROS Organics, 1\%, CAS\# 64-04-0), propanal (ACROS Organics, 1\%, CAS\# 123-38-6), and 1,4diaminobutane (ACROS Organics, 1\%, CAS\# 110-60-1). Odorants were diluted in water or paraffin oil. Odorant cartridges were made by placing a $13 \mathrm{~mm}$ antibiotic assay disc (Whatman) into a Pasteur pipette, pipetting $50 \mu$ odorant onto the disc, and closing the end with a $1 \mathrm{~mL}$ plastic pipette tip. Each odorant cartridge was used a maximum of four times. The tip of the cartridge was inserted into a hole in the main airflow tube, and odorants were applied at $500 \mathrm{~mL} / \mathrm{min}$ for $500 \mathrm{~ms}$. Delivery was controlled via LabChart Pro v8 software (ADInstruments), which directed the opening and closing of a Lee valve (02-21-08i) linked to a ValveBank 4 controller (AutoMate Scientific). Extracellular action potentials were collected with an EXT-02F amplifier (NPI) with a custom 10X head stage. Data were acquired and AC filtered (300-1,700 $\mathrm{Hz}$ ) at $10 \mathrm{kHz}$ with a PowerLab 4/35 digitizer and LabChart Pro v8 software. Spikes were 
1664

1665

1666

1667

1668

1669

1670

1671

1672

1673

1674

1675

1676

1677

1678

1679

1680

1681

1682

1683

1684

1685

1686

1687

1688

summed in coeloconic recordings due to their similar sizes, and they were counted over a 500 ms window, starting at $100 \mathrm{~ms}$ after stimulus onset.

\section{Optogenetics}

Ir25a-T2A-QF2 was crossed to QUAS-CsChrimson \#11C and double balanced to establish a stable stock (Ir25a-T2A-QF2/CyO; QUAS-CsChrimson \#11C/TM6B). Newly eclosed flies (age < 1 day old) were transferred to fly vials containing $0.4 \mathrm{mM}$ all trans-retinal in fly food (SigmaAldrich \#R2500, dissolved in pure DMSO with stock concentration of $0.4 \mathrm{M}$ ). Vials with flies were kept in the dark for at least 4 days before experiments. $627 \mathrm{~nm}$ LED light source (1-up LED Lighting Kit, PART \#: ALK-1UP-EH-KIT) powered by an Arduino Uno

(www.arduino.cc/en/Main/ArduinoBoardUno) was used to activate CsChrimson. By setting the voltage to $2 \mathrm{~V}$ and the distance of the light source to $20 \mathrm{~cm}$ between the LED and the fly antenna, the light intensity was equivalent to $1.13 \mathrm{~W} / \mathrm{m}^{2}$. The antenna was stimulated for 500 ms followed by $5 \mathrm{~s}$ of recovery period for the total recording length of $20 \mathrm{~s}$ ( 3 stimulations). The identity of ab2 and ab3 sensilla were first verified with 1\% ethyl acetate (Sigma \#270989) and 1\% pentyl acetate (Sigma \#109584) before optogenetic experiments. Identification of ab9 sensilla was assisted by fluorescence-guided Single Sensillum Recording (fgSSR) (Lin and Potter, 2015) using Or67b-Gal4 (BDSC \#9995) recombined with 15XUAS-IVS-mCD8::GFP (BDSC \#32193). The $\Delta$ spikes/second was calculated as for other basiconic SSR recordings.

\section{Single-nucleus RNA-sequencing Analyses}

Dataset analyzed in this paper was published in (McLaughlin et al., 2021). The expression levels for the Ir co-receptors across all OSNs were lower than for Orco, even for their corresponding 'canonical' glomeruli. To account for these differences and facilitate comparisons, we performed within-gene normalization in Figure 4-Source Data 1 and used the normalized values to generate the AL maps in Figure 4A. The normalization was performed as follows: first, we determined the fraction of cells within each cluster expressing the given co- 
1689

1690

1691

1692

1693

1694

1695

1696

1697

1698

1699

1700

1701

1702

1703

1704

1705

1706

1707

1708

1709

1710

1711

1712

receptor (read Counts Per Million, CPM threshold $\geq 3$ ). The cluster with the highest fraction value was taken as the maximum. Then, the fraction for each cluster was divided by this maximum value. The normalized value shows the relative strength of expression within each cluster for the given co-receptor gene.

\section{EM Neuron Reconstruction}

VM6 OSNs (Figures 5F-G) were reconstructed in the FAFB EM volume (Zheng et al., 2018) using FlyWire (https://flywire.ai/) (Dorkenwald et al., 2020). Initial candidates were selected based on either being upstream of the VM6 (previously called VC5 in Bates et al., 2020) projection neurons or based on co-fasciculation with already identified VM6 OSNs. Analyses were performed in Python using the open-source packages navis (https://github.com/schlegelp/navis) and fafbseq (https://github.com/flyconnectome/fafbseg-py). OSNs were clustered using FAFB synapse predictions (Buhmann et al., 2021) for a synapsebased NBLAST ("SyNBLAST", implemented in navis). The reconstructions can be viewed at https://flywire.ai/\#links/Task2021a/all.

\section{Quantification and Statistical Analysis}

\section{Cell Counting}

To quantify knock-in co-expression with the corresponding antibodies (Figure 2), the 3D reconstruction software Amira (FEI, Oregon, USA) was used to manually mark individual cell bodies throughout the z-stack in each channel (antibody in far red channel, knock-in in green channel), and the cell markers between channels were compared.

For sacculus cell counts (Figure 5C-E), cells were counted within ImageJ/Fiji using the cell counter tool. Counts were done manually by going through each stack within an image and using different colored markers for each cell type. Cell count data was gathered into an Excel spreadsheet, and analyzed for percent colocalization with $n=9$ antennal sections. 


\section{Statistics}

1714 Statistical analyses on SSR recordings were done in GraphPad Prism (version 8), except for

1715

1716

1717

1718

1719

1720

1721

1722

1723

1724

1725

1726

1727

1728

1729

1730

1731

1732

1733

1734

1735

1736

1737 optogenetic experiments, which were analyzed in Microsoft Excel. Box plots were made using GraphPad Prism; bar graphs were made in Excel. For all analyses, significance level $\alpha=0.05$. The following analyses were performed on all SSR data (excluding optogenetics): within genotype, Kruskal-Wallis test with uncorrected Dunn's to determine which odorant responses were significantly different from mineral oil or paraffin oil control; between genotype, MannWhitney $U$ to compare responses of two genotypes to the same odorant (e.g. wildtype vs Ir25a ${ }^{2}$ mutant, or wildtype versus Orco-T2A-QF2 knock-in). Summary tables in Figure 6 are filled in based on the following criteria: no response means neither wildtype nor $\operatorname{lr} 25 \mathrm{a}^{2}$ mutant odorevoked activity for given odorant was significantly different from its respective mineral oil control, nor was the difference between the genotypes statistically significant; no difference means that either wildtype or mutant or both had a significantly different odor-evoked response to the stimulus compared to mineral oil control, but the difference between the two genotypes was not statistically significant; higher response (in either wildtype or mutant) means that there was a statistically significant difference between genotypes for the given odorant. This could mean that: a) one genotype did not have a response, while the other did; b) both genotypes had a response, and one was higher; c) responses are different from each other, but not from their respective mineral oil controls; or d) neural activity was inhibited by the odorant in one genotype compared to mineral oil control, and either not inhibited in the other genotype or inhibited to a lesser degree. Non-parametric tests were chosen due to small sample sizes and/or data that were not normally distributed.

In Figure 6I, stimulus responses which were statistically significantly different from mineral oil control were those whose $\Delta$ spike values were zero, due to the fact that the mineral oil control $\Delta$ spike value was non-zero (median $=1.2$, range $=0$ to 2$)$. Because of this, we did 
bioRxiv preprint doi: https://doi.org/10.1101/2020.11.07.355651; this version posted July 27, 2021. The copyright holder for this preprint (which was not certified by peer review) is the author/funder. All rights reserved. No reuse allowed without permission.

1738 not deem these differences as biologically relevant. Nevertheless, $p$ values are reported in the 1739 figure legend of Figure 6, and detailed information can be found in Figure 6-Source Data 1. 1740 


\section{References}

1742

1743

1744

1745

1746

1747

1748

1749

1750

1751

1752

1753

1754

1755

1756

1757

1758

1759

1760

1761

1762

1763

1764

1765

1766

1767

1768

1769

1770

1771

1772

1773

1774

1775

1776

1777

1778

1779

1780

1781

1782

Abuin, L., Bargeton, B., Ulbrich, M.H., Isacoff, E.Y., Kellenberger, S., and Benton, R. (2011). Functional architecture of olfactory ionotropic glutamate receptors. Neuron 69, 44-60.

Abuin, L., Prieto-Godino, L.L., Pan, H., Gutierrez, C., Huang, L., Jin, R., and Benton, R. (2019). In vivo assembly and trafficking of olfactory lonotropic Receptors. BMC Biol 17, 34.

Ache, B.W., and Young, J.M. (2005). Olfaction: diverse species, conserved principles. Neuron 48, 417430.

Ai, M., Blais, S., Park, J.Y., Min, S., Neubert, T.A., and Suh, G.S. (2013). lonotropic glutamate receptors IR64a and IR8a form a functional odorant receptor complex in vivo in Drosophila. J Neurosci 33, 10741-10749.

Ai, M., Min, S., Grosjean, Y., Leblanc, C., Bell, R., Benton, R., and Suh, G.S. (2010). Acid sensing by the Drosophila olfactory system. Nature 468, 691-695.

Auer, T.O., Khallaf, M.A., Silbering, A.F., Zappia, G., Ellis, K., Alvarez-Ocana, R., Arguello, J.R., Hansson, B.S., Jefferis, G., Caron, S.J.C., et al. (2020). Olfactory receptor and circuit evolution promote host specialization. Nature 579, 402-408.

Awasaki, T., Huang, Y., O'Connor, M.B., and Lee, T. (2011). Glia instruct developmental neuronal remodeling through TGF- $\beta$ signaling. Nat Neurosci 14, 821-823.

Baena-Lopez, L.A., Alexandre, C., Mitchell, A., Pasakarnis, L., and Vincent, J.P. (2013). Accelerated homologous recombination and subsequent genome modification in Drosophila. Development 140, 4818-4825.

Bates, A.S., Schlegel, P., Roberts, R.J.V., Drummond, N., Tamimi, I.F.M., Turnbull, R., Zhao, X., Marin, E.C., Popovici, P.D., Dhawan, S., et al. (2020). Complete Connectomic Reconstruction of Olfactory Projection Neurons in the Fly Brain. Curr Biol 30, 3183-3199.e3186.

Benton, R., Sachse, S., Michnick, S.W., and Vosshall, L.B. (2006). Atypical membrane topology and heteromeric function of Drosophila odorant receptors in vivo. PLoS Biol 4, e20.

Benton, R., Vannice, K.S., Gomez-Diaz, C., and Vosshall, L.B. (2009). Variant ionotropic glutamate receptors as chemosensory receptors in Drosophila. Cell 136, 149-162.

Berck, M.E., Khandelwal, A., Claus, L., Hernandez-Nunez, L., Si, G., Tabone, C.J., Li, F., Truman, J.W., Fetter, R.D., Louis, M., et al. (2016). The wiring diagram of a glomerular olfactory system. elife 5.

Bhalerao, S., Sen, A., Stocker, R., and Rodrigues, V. (2003). Olfactory neurons expressing identified receptor genes project to subsets of glomeruli within the antennal lobe of Drosophila melanogaster. J Neurobiol 54, 577-592.

Bischof, J., Maeda, R.K., Hediger, M., Karch, F., and Basler, K. (2007). An optimized transgenesis system for Drosophila using germ-line-specific phiC31 integrases. PNAS 104, 3312-3317.

Bosch, J.A., Knight, S., Kanca, O., Zirin, J., Yang-Zhou, D., Hu, Y., Rodiger, J., Amador, G., Bellen, H.J., Perrimon, N., et al. (2020). Use of the CRISPR-Cas9 System in Drosophila Cultured Cells to Introduce Fluorescent Tags into Endogenous Genes. Curr Protoc Mol Biol 130, e112.

Brand, A.H., and Perrimon, N. (1993). Targeted gene expression as a means of altering cell fates and generating dominant phenotypes. Development 118, 401-415.

Budelli, G., Ni, L., Berciu, C., van Giesen, L., Knecht, Z.A., Chang, E.C., Kaminski, B., Silbering, A.F., Samuel, A., Klein, M., et al. (2019). Ionotropic Receptors Specify the Morphogenesis of Phasic Sensors Controlling Rapid Thermal Preference in Drosophila. Neuron 101, 738-747 e733. 
Buhmann, J., Sheridan, A., Malin-Mayor, C., Schlegel, P., Gerhard, S., Kazimiers, T., Krause, R., Nguyen, T.M., Heinrich, L., Lee, W.-C.A., et al. (2021). Automatic detection of synaptic partners in a whole-brain Drosophila electron microscopy data set. Nat Methods 18, 771-774.

Butterwick, J.A., Del Marmol, J., Kim, K.H., Kahlson, M.A., Rogow, J.A., Walz, T., and Ruta, V. (2018). Cryo-EM structure of the insect olfactory receptor Orco. Nature 560, 447-452.

Carey, A.F., and Carlson, J.R. (2011). Insect olfaction from model systems to disease control. PNAS 108, 12987-12995.

Chai, P.C., Cruchet, S., Wigger, L., and Benton, R. (2019). Sensory neuron lineage mapping and manipulation in the Drosophila olfactory system. Nat Commun 10, 643.

Chen, C., Buhl, E., Xu, M., Croset, V., Rees, J.S., Lilley, K.S., Benton, R., Hodge, J.J., and Stanewsky, R. (2015). Drosophila lonotropic Receptor 25a mediates circadian clock resetting by temperature. Nature 527, 516-520.

Chen, H.-M., Yao, X., Ren, Q., Chang, C.-C., Liu, L.-Y., Miyares, R.L., and Lee, T. (2020). Enhanced Golic+: highly effective CRISPR gene targeting and transgene HACKing in Drosophila. Development 147.

Chen, Y., and Amrein, H. (2017). Ionotropic Receptors Mediate Drosophila Oviposition Preference Through Sour Gustatory Receptor Neurons. Curr Biol 27, 2741-2750 e2744.

Chen, Y.D., and Dahanukar, A. (2017). Molecular and Cellular Organization of Taste Neurons in Adult Drosophila Pharynx. Cell Rep 21, 2978-2991.

Choi, H.M.T., Calvert, C.R., Husain, N., Huss, D., Barsi, J.C., Deverman, B.E., Hunter, R.C., Kato, M., Lee, S.M., Abelin, A.C.T., et al. (2016). Mapping a multiplexed zoo of mRNA expression. Development 143, 3632-3637.

Choi, H.M.T., Schwarzkopf, M., Fornace, M.E., Acharya, A., Artavanis, G., Stegmaier, J., Cunha, A., and Pierce, N.A. (2018). Third-generation in situ hybridization chain reaction: multiplexed, quantitative, sensitive, versatile, robust. Development 145.

Clyne, P.J., Warr, C.G., Freeman, M.R., Lessing, D., Kim, J., and Carlson, J.R. (1999). A novel family of divergent seven-transmembrane proteins: candidate odorant receptors in Drosophila. Neuron 22, 327-338.

Couto, A., Alenius, M., and Dickson, B.J. (2005). Molecular, anatomical, and functional organization of the Drosophila olfactory system. Curr Biol 15, 1535-1547.

Croset, V., Rytz, R., Cummins, S.F., Budd, A., Brawand, D., Kaessmann, H., Gibson, T.J., and Benton, R. (2010). Ancient protostome origin of chemosensory ionotropic glutamate receptors and the evolution of insect taste and olfaction. PLoS Genet 6, e1001064.

de Bruyne, M., Clyne, P.J., and Carlson, J.R. (1999). Odor Coding in a Model Olfactory Organ: The Drosophila Maxillary Palp. J Neurosci 19, 4520-4532.

de Bruyne, M., Foster, K., and Carlson, J.R. (2001). Odor Coding in the Drosophila Antenna. Neuron 30, 537-552.

DeGennaro, M., McBride, C.S., Seeholzer, L., Nakagawa, T., Dennis, E.J., Goldman, C., Jasinskiene, N., James, A.A., and Vosshall, L.B. (2013). orco mutant mosquitoes lose strong preference for humans and are not repelled by volatile DEET. Nature 498, 487-491.

Depetris-Chauvin, A., Galagovsky, D., and Grosjean, Y. (2015). Chemicals and chemoreceptors: ecologically relevant signals driving behavior in Drosophila. Front Ecol Evol 3, 41. 
Diao, F., Ironfield, H., Luan, H., Diao, F., Shropshire, W.C., Ewer, J., Marr, E., Potter, C.J., Landgraf, M., and White, B.H. (2015). Plug-and-play genetic access to Drosophila cell types using exchangeable exon cassettes. Cell Rep 10, 1410-1421.

Diao, F., and White, B.H. (2012). A novel approach for directing transgene expression in Drosophila: T2AGal4 in-frame fusion. Genetics 190, 1139-1144.

Dorkenwald, S., McKellar, C., Macrina, T., Kemnitz, N., Lee, K., Lu, R., Wu, J., Popovych, S., Mitchell, E., Nehoran, B., et al. (2020). FlyWire: Online community for whole-brain connectomics. bioRxiv, 2020.2008.2030.274225.

Du, L., Zhou, A., Sohr, A., and Roy, S. (2018). An Efficient Strategy for Generating Tissue-specific Binary Transcription Systems in Drosophila by Genome Editing. J Vis Exp.

Dunipace, L., Meister, S., McNealy, C., and Amrein, H. (2001). Spatially restricted expression of candidate taste receptors in the Drosophila gustatory system. Curr Biol 11, 822-835.

Dweck, H.K.M., Ebrahim, S.A.M., Thoma, M., Mohamed, A.A.M., Keesey, I.W., Trona, F., Lavista-Llanos, S., Svatoš, A., Sachse, S., Knaden, M., et al. (2015). Pheromones mediating copulation and attraction in Drosophila. PNAS 112, E2829-E2835.

Endo, K., Aoki, T., Yoda, Y., Kimura, K.-i., and Hama, C. (2007). Notch signal organizes the Drosophila olfactory circuitry by diversifying the sensory neuronal lineages. Nat Neurosci 10, 153-160.

Enjin, A., Zaharieva, E.E., Frank, D.D., Mansourian, S., Suh, G.S., Gallio, M., and Stensmyr, M.C. (2016). Humidity Sensing in Drosophila. Curr Biol 26, 1352-1358.

Fandino, R.A., Haverkamp, A., Bisch-Knaden, S., Zhang, J., Bucks, S., Nguyen, T.A.T., Schroder, K., Werckenthin, A., Rybak, J., Stengl, M., et al. (2019). Mutagenesis of odorant coreceptor Orco fully disrupts foraging but not oviposition behaviors in the hawkmoth Manduca sexta. PNAS 116, 1567715685.

Fishilevich, E., and Vosshall, L.B. (2005). Genetic and functional subdivision of the Drosophila antennal lobe. Curr Biol 15, 1548-1553.

Frank, D.D., Enjin, A., Jouandet, G.C., Zaharieva, E.E., Para, A., Stensmyr, M.C., and Gallio, M. (2017). Early Integration of Temperature and Humidity Stimuli in the Drosophila Brain. Curr Biol 27, 2381-2388 e2384.

Frechter, S., Bates, A.S., Tootoonian, S., Dolan, M.J., Manton, J., Jamasb, A.R., Kohl, J., Bock, D., and Jefferis, G. (2019). Functional and anatomical specificity in a higher olfactory centre. eLife 8.

Fujii, S., Yavuz, A., Slone, J., Jagge, C., Song, X., and Amrein, H. (2015). Drosophila sugar receptors in sweet taste perception, olfaction, and internal nutrient sensing. Curr Biol 25, 621-627.

Ganguly, A., Pang, L., Duong, V.K., Lee, A., Schoniger, H., Varady, E., and Dahanukar, A. (2017). A Molecular and Cellular Context-Dependent Role for Ir76b in Detection of Amino Acid Taste. Cell Rep 18, 737-750.

Gao, Q., and Chess, A. (1999). Identification of candidate Drosophila olfactory receptors from genomic DNA sequence. Genomics 60, 31-39.

Gao, Q., Yuan, B., and Chess, A. (2000). Convergent projections of Drosophila olfactory neurons to specific glomeruli in the antennal lobe. Nat Neurosci 3, 780-785.

Gloor, G.B., Nassif, N.A., Johnson-Schlitz, D.M., Preston, C.R., and Engels, W.R. (1991). Targeted gene replacement in Drosophila via P element-induced gap repair. Science 253, 1110-1117. 
Gnerer, J.P., Venken, K.J., and Dierick, H.A. (2015). Gene-specific cell labeling using MiMIC transposons. Nucleic Acids Res 43, e56.

Goldman, A.L., Van der Goes van Naters, W., Lessing, D., Warr, C.G., and Carlson, J.R. (2005). Coexpression of two functional odor receptors in one neuron. Neuron 45, 661-666.

Gomez-Diaz, C., Martin, F., Garcia-Fernandez, J.M., and Alcorta, E. (2018). The Two Main Olfactory Receptor Families in Drosophila, ORs and IRs: A Comparative Approach. Front Cell Neurosci 12, 253.

Goulding, S.E., zur Lage, P., and Jarman, A.P. (2000). amos, a Proneural Gene for Drosophila Olfactory Sense Organs that Is Regulated by lozenge. Neuron 25, 69-78.

Grabe, V., Baschwitz, A., Dweck, H.K.M., Lavista-Llanos, S., Hansson, B.S., and Sachse, S. (2016). Elucidating the Neuronal Architecture of Olfactory Glomeruli in the Drosophila Antennal Lobe. Cell Rep 16, 3401-3413.

Grabe, V., and Sachse, S. (2018). Fundamental principles of the olfactory code. Biosystems 164, 94-101.

Gratz, S.J., Ukken, F.P., Rubinstein, C.D., Thiede, G., Donohue, L.K., Cummings, A.M., and O'Connor-Giles, K.M. (2014). Highly specific and efficient CRISPR/Cas9-catalyzed homology-directed repair in Drosophila. Genetics 196, 961-971.

Groth, A.C., Fish, M., Nusse, R., and Calos, M.P. (2004). Construction of transgenic Drosophila by using the site-specific integrase from phage phiC31. Genetics 166, 1775-1782.

Gupta, B.P., and Rodrigues, V. (1997). Atonal is a proneural gene for a subset of olfactory sense organs in Drosophila. Genes Cells 2, 225-233.

Hallem, E.A., and Carlson, J.R. (2006). Coding of odors by a receptor repertoire. Cell 125, 143-160.

Hallem, E.A., Ho, M.G., and Carlson, J.R. (2004). The molecular basis of odor coding in the Drosophila antenna. Cell 117, 965-979.

Hansson, B.S., and Stensmyr, M.C. (2011). Evolution of insect olfaction. Neuron 72, 698-711.

Harris, W.A. (1972). The maxillae of Drosophila melanogaster as revealed by scanning electron microscopy. J Morphol 138, 451-456.

Haverkamp, A., Hansson, B.S., and Knaden, M. (2018). Combinatorial Codes and Labeled Lines: How Insects Use Olfactory Cues to Find and Judge Food, Mates, and Oviposition Sites in Complex Environments. Front Physiol 9, 49.

Horne, J.A., Langille, C., McLin, S., Wiederman, M., Lu, Z., Xu, C.S., Plaza, S.M., Scheffer, L.K., Hess, H.F., and Meinertzhagen, I.A. (2018). A resource for the Drosophila antennal lobe provided by the connectome of glomerulus VA1v. eLife 7.

Hussain, A., Zhang, M., Ucpunar, H.K., Svensson, T., Quillery, E., Gompel, N., Ignell, R., and Grunwald Kadow, I.C. (2016). Ionotropic Chemosensory Receptors Mediate the Taste and Smell of Polyamines. PLoS Biol 14, e1002454.

Jaeger, A.H., Stanley, M., Weiss, Z.F., Musso, P.Y., Chan, R.C., Zhang, H., Feldman-Kiss, D., and Gordon, M.D. (2018). A complex peripheral code for salt taste in Drosophila. eLife 7.

Jhaveri, D., Sen, A., Reddy, G.V., and Rodrigues, V. (2000a). Sense organ identity in the Drosophila antenna is specified by the expression of the proneural gene atonal. Mech Dev 99, 101-111.

Jhaveri, D., Sen, A., and Rodrigues, V. (2000b). Mechanisms underlying olfactory neuronal connectivity in Drosophila-the atonal lineage organizes the periphery while sensory neurons and glia pattern the olfactory lobe. Dev Biol 226, 73-87. 
1906

1907

1908

1909

1910

1911

1912

1913

1914

1915

1916

1917

1918

1919

1920

1921

1922

1923

1924

1925

1926

1927

1928

1929

1930

1931

1932

1933

1934

1935

1936

1937

1938

1939

1940

1941

1942

1943

1944

1945

Jinek, M., Chylinski, K., Fonfara, I., Hauer, M., Doudna, J.A., and Charpentier, E. (2012). A programmable dual-RNA-guided DNA endonuclease in adaptive bacterial immunity. Science 337, 816-821.

Jones, W.D., Cayirlioglu, P., Kadow, I.G., and Vosshall, L.B. (2007). Two chemosensory receptors together mediate carbon dioxide detection in Drosophila. Nature 445, 86-90.

Kaissling, K.-E., and Thorson, J. (1980). Insect olfactory sensilla: structural, chemical and electrical aspects of the functional organisation. In Receptors for neurotransmitters, hormones and pheromones in insects (Elsevier/North-Holland Biomedical Press), pp. 261-282.

Kanca, O., Zirin, J., Garcia-Marques, J., Knight, S.M., Yang-Zhou, D., Amador, G., Chung, H., Zuo, Z., Ma, L., He, Y., et al. (2019). An efficient CRISPR-based strategy to insert small and large fragments of DNA using short homology arms. eLife 8, e51539.

Karim, M.R., Endo, K., Moore, A.W., and Taniguchi, H. (2014). Whole mount immunolabeling of olfactory receptor neurons in the Drosophila antenna. J Vis Exp, 51245.

Klapoetke, N.C., Murata, Y., Kim, S.S., Pulver, S.R., Birdsey-Benson, A., Cho, Y.K., Morimoto, T.K., Chuong, A.S., Carpenter, E.J., Tian, Z., et al. (2014). Independent optical excitation of distinct neural populations. Nat Methods 11, 338-346.

Knecht, Z.A., Silbering, A.F., Cruz, J., Yang, L., Croset, V., Benton, R., and Garrity, P.A. (2017). lonotropic Receptor-dependent moist and dry cells control hygrosensation in Drosophila. elife 6.

Knecht, Z.A., Silbering, A.F., Ni, L., Klein, M., Budelli, G., Bell, R., Abuin, L., Ferrer, A.J., Samuel, A.D., Benton, R., et al. (2016). Distinct combinations of variant ionotropic glutamate receptors mediate thermosensation and hygrosensation in Drosophila. elife 5.

Koh, T.W., He, Z., Gorur-Shandilya, S., Menuz, K., Larter, N.K., Stewart, S., and Carlson, J.R. (2014). The Drosophila IR20a clade of ionotropic receptors are candidate taste and pheromone receptors. Neuron 83, 850-865.

Kondoh, K., Lu, Z., Ye, X., Olson, D.P., Lowell, B.B., and Buck, L.B. (2016). A specific area of olfactory cortex involved in stress hormone responses to predator odours. Nature 532, 103-106.

Kurtovic, A., Widmer, A., and Dickson, B.J. (2007). A single class of olfactory neurons mediates behavioural responses to a Drosophila sex pheromone. Nature 446, 542-546.

Kwon, J.Y., Dahanukar, A., Weiss, L.A., and Carlson, J.R. (2007). The molecular basis of CO2 reception in Drosophila. PNAS 104, 3574-3578.

Lai, S.-L., and Lee, T. (2006). Genetic mosaic with dual binary transcriptional systems in Drosophila. Nat Neurosci 9, 703-709.

Laissue, P.P., Reiter, C.H., Hiesinger, P.R., Halter, S., Fischbach, K.F., and Stocker, R.F. (1999). Threedimensional reconstruction of the antennal lobe in Drosophila melanogaster. J Comp Neurol 405, 543552.

Larsson, M.C., Domingos, A.I., Jones, W.D., Chiappe, M.E., Amrein, H., and Vosshall, L.B. (2004). Or83b encodes a broadly expressed odorant receptor essential for Drosophila olfaction. Neuron 43, 703-714.

Lee, P.-T., Zirin, J., Kanca, O., Lin, W.-W., Schulze, K.L., Li-Kroeger, D., Tao, R., Devereaux, C., Hu, Y., Chung, V., et al. (2018). A gene-specific T2A-GAL4 library for Drosophila. eLife 7, e35574.

Lee, T., and Luo, L. (1999). Mosaic Analysis with a Repressible Cell Marker for Studies of Gene Function in Neuronal Morphogenesis. Neuron 22, 451-461. 
1946

1947

1948

1949

1950

1951

1952

1953

1954

1955

1956

1957

1958

1959

1960

1961

1962

1963

1964

1965

1966

1967

1968

1969

1970

1971

1972

1973

1974

1975

1976

1977

1978

1979

1980

1981

1982

1983

1984

1985

1986

1987

Li-Kroeger, D., Kanca, O., Lee, P.T., Cowan, S., Lee, M.T., Jaiswal, M., Salazar, J.L., He, Y., Zuo, Z., and Bellen, H.J. (2018). An expanded toolkit for gene tagging based on MiMIC and scarless CRISPR tagging in Drosophila. eLife 7.

Li, H., Janssens, J., De Waegeneer, M., Kolluru, S.S., Davie, K., Gardeux, V., Sealens, W., David, F., Brbic, M., Leskovec, J., et al. (2021). Fly Cell Atlas: a single-cell transcriptomic atlas of the adult fruit fly. bioRxiv, 2021.2007.2004.451050.

Li, Q., Barish, S., Okuwa, S., Maciejewski, A., Brandt, A.T., Reinhold, D., Jones, C.D., and Volkan, P.C. (2016). A Functionally Conserved Gene Regulatory Network Module Governing Olfactory Neuron Diversity. In PLoS Genet, pp. e1005780.

Lin, C.-C., and Potter, C.J. (2015). Re-Classification of Drosophila melanogaster Trichoid and Intermediate Sensilla Using Fluorescence-Guided Single Sensillum Recording. PLoS One 10, e0139675.

Lin, C.-C., and Potter, C.J. (2016a). Editing Transgenic DNA Components by Inducible Gene Replacement in Drosophila melanogaster. Genetics 203, 1613-1628.

Lin, C.-C., and Potter, C.J. (2016b). Non-mendelian dominant maternal effects caused by CRISPR/Cas9 transgenic components in Drosophila melanogaster. G3 (Bethesda) 6, 3685-3691.

MacWilliam, D., Kowalewski, J., Kumar, A., Pontrello, C., and Ray, A. (2018). Signaling Mode of the Broad-Spectrum Conserved CO2 Receptor Is One of the Important Determinants of Odor Valence in Drosophila. Neuron 97, 1153-1167.e1154.

Mansourian, S., Corcoran, J., Enjin, A., Löfstedt, C., Dacke, M., and Stensmyr, M.C. (2016). Fecal-Derived Phenol Induces Egg-Laying Aversion in Drosophila. Curr Biol 26, 2762-2769.

Marin, E.C., Büld, L., Theiss, M., Sarkissian, T., Roberts, R.J.V., Turnbull, R., Tamimi, I.F.M., Pleijzier, M.W., Laursen, W.J., Drummond, N., et al. (2020). Connectomics Analysis Reveals First-, Second-, and ThirdOrder Thermosensory and Hygrosensory Neurons in the Adult Drosophila Brain. Curr Biol 30, 31673182.e3164.

McLaughlin, C.N., Brbić, M., Xie, Q., Li, T., Horns, F., Kolluru, S.S., Kebschull, J.M., Vacek, D., Xie, A., Li, J., et al. (2021). Single-cell transcriptomes of developing and adult olfactory receptor neurons in Drosophila. eLife 10, e63856.

Menuz, K., Larter, N.K., Park, J., and Carlson, J.R. (2014). An RNA-seq screen of the Drosophila antenna identifies a transporter necessary for ammonia detection. PLoS Genet 10, e1004810.

Min, S., Ai, M., Shin, S.A., and Suh, G.S. (2013). Dedicated olfactory neurons mediating attraction behavior to ammonia and amines in Drosophila. PNAS 110, E1321-1329.

Papes, F., Logan, D.W., and Stowers, L. (2010). The Vomeronasal Organ Mediates Interspecies Defensive Behaviors through Detection of Protein Pheromone Homologs. Cell 141, 692-703.

Park, J.H., and Kwon, J.Y. (2011). A systematic analysis of Drosophila gustatory receptor gene expression in abdominal neurons which project to the central nervous system. Mol Cells 32, 375-381.

Pask, G.M., and Ray, A. (2016). Insect Olfactory Receptors: An Interface between Chemistry and Biology. In Chemosensory Transduction (Elsevier), pp. 101-122.

Pinto, L., Stocker, R.F., and Rodrigues, V. (1988). Anatomical and neurochemical classification of the antennal glomeruli in Drosophila melanogaster meigen (Diptera: Drosophilidae). Int J Insect Morphol Embryol 17, 335-344.

Port, F., Chen, H.M., Lee, T., and Bullock, S.L. (2014). Optimized CRISPR/Cas tools for efficient germline and somatic genome engineering in Drosophila. PNAS 111, E2967-2976. 
Potter, C.J., Tasic, B., Russler, E.V., Liang, L., and Luo, L. (2010). The Q system: a repressible binary system for transgene expression, lineage tracing, and mosaic analysis. Cell 141, 536-548.

Prieto-Godino, L.L., Rytz, R., Cruchet, S., Bargeton, B., Abuin, L., Silbering, A.F., Ruta, V., Dal Peraro, M., and Benton, R. (2017). Evolution of Acid-Sensing Olfactory Circuits in Drosophilids. Neuron 93, 661676.e666.

Prieto-Godino, L.L., Silbering, A.F., Khallaf, M.A., Cruchet, S., Bojkowska, K., Pradervand, S., Hansson, B.S., Knaden, M., and Benton, R. (2020). Functional integration of "undead" neurons in the olfactory system. Sci Adv 6, eaaz7238.

Ray, A., Van der Goes van Naters, W., and Carlson, J.R. (2008). A regulatory code for neuron-specific odor receptor expression. PLoS Biol 6, e125.

Ray, A., Van der Goes van Naters, W., Shiraiwa, T., and Carlson, J.R. (2007). Mechanisms of odor receptor gene choice in Drosophila. Neuron 53, 353-369.

Riabinina, O., Luginbuhl, D., Marr, E., Liu, S., Wu, M.N., Luo, L., and Potter, C.J. (2015). Improved and expanded Q-system reagents for genetic manipulations. Nat Methods 12, 219-222, 215 p following 222.

Riabinina, O., Task, D., Marr, E., Lin, C.-C., Alford, R., O'Brochta, D.A., and Potter, C.J. (2016). Organization of olfactory centres in the malaria mosquito Anopheles gambiae. Nat Commun 7, 13010.

Saina, M., and Benton, R. (2013). Visualizing Olfactory Receptor Expression and Localization in Drosophila. In Olfactory Receptors: Methods and Protocols, C.J. Crasto, ed. (Totowa, NJ: Humana Press), pp. 211-228.

Sanchez-Alcaniz, J.A., Silbering, A.F., Croset, V., Zappia, G., Sivasubramaniam, A.K., Abuin, L., Sahai, S.Y., Munch, D., Steck, K., Auer, T.O., et al. (2018). An expression atlas of variant ionotropic glutamate receptors identifies a molecular basis of carbonation sensing. Nat Commun 9, 4252.

Sato, K., Pellegrino, M., Nakagawa, T., Nakagawa, T., Vosshall, L.B., and Touhara, K. (2008). Insect olfactory receptors are heteromeric ligand-gated ion channels. Nature 452, 1002-1006.

Scheffer, L.K., Xu, C.S., Januszewski, M., Lu, Z., Takemura, S.-y., Hayworth, K.J., Huang, G.B., Shinomiya, K., Maitlin-Shepard, J., Berg, S., et al. (2020). A connectome and analysis of the adult Drosophila central brain. eLife 9, e57443.

Schindelin, J., Arganda-Carreras, I., Frise, E., Kaynig, V., Longair, M., Pietzsch, T., Preibisch, S., Rueden, C., Saalfeld, S., and Schmid, B. (2012). Fiji: an open-source platform for biological-image analysis. Nat Methods 9, 676-682.

Schlegel, P., Bates, A.S., Stürner, T., Jagannathan, S.R., Drummond, N., Hsu, J., Serratosa Capdevila, L., Javier, A., Marin, E.C., Barth-Maron, A., et al. (2021). Information flow, cell types and stereotypy in a full olfactory connectome. eLife 10, e66018.

Scott, K. (2018). Gustatory Processing in Drosophila melanogaster. Annu Rev Entomol 63, 15-30.

Scott, K., Brady, R., Cravchik, A., Morozov, P., Rzhetsky, A., Zuker, C., and Axel, R. (2001). A Chemosensory Gene Family Encoding Candidate Gustatory and Olfactory Receptors in Drosophila. Cell 104, 661-673.

Seki, Y., Dweck, H.K.M., Rybak, J., Wicher, D., Sachse, S., and Hansson, B.S. (2017). Olfactory coding from the periphery to higher brain centers in the Drosophila brain. BMC Biol 15, 56.

Shearin, H.K., Macdonald, I.S., Spector, L.P., and Stowers, R.S. (2014). Hexameric GFP and mCherry reporters for the Drosophila GAL4, Q, and LexA transcription systems. Genetics 196, 951-960. 
Siddiqi, O. (1987). Neurogenetics of olfaction in Drosophila melanogaster. Trends Genet 3, 137-142.

Siegal, M.L., and Hartl, D.L. (1996). Transgene Coplacement and high efficiency site-specific recombination with the Cre/loxP system in Drosophila. Genetics 144, 715-726.

Silbering, A.F., Rytz, R., Grosjean, Y., Abuin, L., Ramdya, P., Jefferis, G.S., and Benton, R. (2011). Complementary function and integrated wiring of the evolutionarily distinct Drosophila olfactory subsystems. J Neurosci 31, 13357-13375.

Spletter, M.L., Liu, J., Liu, J., Su, H., Giniger, E., Komiyama, T., Quake, S., and Luo, L. (2007). Lola regulates Drosophila olfactory projection neuron identity and targeting specificity. Neural Dev 2, 14.

Stengl, M. (2010). Pheromone transduction in moths. Front Cell Neurosci 4, 133-133.

Stensmyr, M.C., Dweck, H.K., Farhan, A., Ibba, I., Strutz, A., Mukunda, L., Linz, J., Grabe, V., Steck, K., Lavista-Llanos, S., et al. (2012). A Conserved Dedicated Olfactory Circuit for Detecting Harmful Microbes in Drosophila. Cell 151, 1345-1357.

Stocker, R.F. (1994). The organization of the chemosensory system in Drosophila melanogaster: a review. Cell Tissue Res 275, 3-26.

Stocker, R.F. (2001). Drosophila as a focus in olfactory research: mapping of olfactory sensilla by fine structure, odor specificity, odorant receptor expression, and central connectivity. Microsc Res Tech 55, 284-296.

Su, C.Y., Menuz, K., and Carlson, J.R. (2009). Olfactory perception: receptors, cells, and circuits. Cell 139, 45-59.

Suh, G.S.B., Wong, A.M., Hergarden, A.C., Wang, J.W., Simon, A.F., Benzer, S., Axel, R., and Anderson, D.J. (2004). A single population of olfactory sensory neurons mediates an innate avoidance behaviour in Drosophila. Nature 431, 854-859.

2052

Task, D., Lin, C.-C., Afify, A., Li, H., Vulpe, A., Menuz, K., and Potter, C.J. (2020). Widespread Polymodal Chemosensory Receptor Expression in Drosophila Olfactory Neurons. bioRxiv, 2020.2011.2007.355651.

Task, D., and Potter, C.J. (2021). Rapid degeneration of Drosophila olfactory neurons in Orco mutant maxillary palps. microPublication Biology.

Thurmond, J., Goodman, J.L., Strelets, V.B., Attrill, H., Gramates, L.S., Marygold, S.J., Matthews, B.B., Millburn, G., Antonazzo, G., and Trovisco, V. (2019). FlyBase 2.0: the next generation. Nucleic Acids Res 47, D759-D765.

2060

2061

2062

2063

2064

2065

2066

2067

2068

Trible, W., Olivos-Cisneros, L., McKenzie, S.K., Saragosti, J., Chang, N.-C., Matthews, B.J., Oxley, P.R., and Kronauer, D.J.C. (2017). Orco mutagenesis causes loss of antennal lobe glomeruli and impaired social behavior in ants. Cell 170, 727-735. e710.

van Breugel, F., Huda, A., and Dickinson, M.H. (2018). Distinct activity-gated pathways mediate attraction and aversion to $\mathrm{CO} 2$ in Drosophila. Nature 564, 420-424.

Van der Maaten, L., and Hinton, G. (2008). Visualizing data using t-SNE. J Mach Learn Res 9.

Venkatesh, S., and Singh, R.N. (1984). Sensilla on the third antennal segment of Drosophila melanogaster meigen (Diptera : Drosophilidae). Int J Insect Morphol Embryol 13, 51-63.

Vilain, S., Vanhauwaert, R., Maes, I., Schoovaerts, N., Zhou, L., Soukup, S., da Cunha, R., Lauwers, E., Fiers, M., and Verstreken, P. (2014). Fast and efficient Drosophila melanogaster gene knock-ins using MiMIC transposons. G3 (Bethesda) 4, 2381-2387. 
2071

2072

2073

2074

2075

2076

2077

2078

2079

2080

2081

2082

2083

2084

2085

2086

2087

2088

2089

2090

2091

2092

2093

2094

2095

2096

2097

2098

2099

2100

2101

2102

2103

2104

2105

2106

2107

Vosshall, L.B., Amrein, H., Morozov, P.S., Rzhetsky, A., and Axel, R. (1999). A Spatial Map of Olfactory Receptor Expression in the Drosophila Antenna. Cell 96, 725-736.

Vosshall, L.B., and Hansson, B.S. (2011). A unified nomenclature system for the insect olfactory coreceptor. Chem Senses 36, 497-498.

Vosshall, L.B., and Stocker, R.F. (2007). Molecular architecture of smell and taste in Drosophila. Annu Rev Neurosci 30, 505-533.

Vosshall, L.B., Wong, A.M., and Axel, R. (2000). An Olfactory Sensory Map in the Fly Brain. Cell 102, 147159.

Vulpe, A., Kim, H.S., Ballou, S., Wu, S.-T., Grabe, V., Nava Gonzales, C., Liang, T., Sachse, S., Jeanne, J.M., Su, C.-Y., et al. (2021a). An ammonium transporter is a non-canonical olfactory receptor for ammonia. Curr Biol.

Vulpe, A., Mohapatra, P., and Menuz, K. (2021b). Functional characterization of odor responses and gene expression changes in olfactory co-receptor mutants in Drosophila. bioRxiv, 2021.2006.2018.449017.

Wilson, R.I. (2013). Early olfactory processing in Drosophila: mechanisms and principles. Annu Rev Neurosci 36, 217-241.

Xie, T., Ho, M.C.W., Liu, Q., Horiuchi, W., Lin, C.-C., Task, D., Luan, H., White, B.H., Potter, C.J., and Wu, M.N. (2018). A genetic toolkit for dissecting dopamine circuit function in Drosophila. Cell Rep 23, 652665.

Xue, Z., Ren, M., Wu, M., Dai, J., Rong, Y.S., and Gao, G. (2014). Efficient gene knock-out and knock-in with transgenic Cas9 in Drosophila. G3 (Bethesda) 4, 925-929.

Yan, H., Jafari, S., Pask, G., Zhou, X., Reinberg, D., and Desplan, C. (2020). Evolution, developmental expression and function of odorant receptors in insects. J Exp Biol 223.

Yan, H., Opachaloemphan, C., Mancini, G., Yang, H., Gallitto, M., Mlejnek, J., Leibholz, A., Haight, K., Ghaninia, M., Huo, L., et al. (2017). An Engineered orco Mutation Produces Aberrant Social Behavior and Defective Neural Development in Ants. Cell 170, 736-747.e739.

Yao, C.A., Ignell, R., and Carlson, J.R. (2005). Chemosensory Coding by Neurons in the Coeloconic Sensilla of the Drosophila Antenna. J Neurosci 25, 8359-8367.

Younger, M.A., Herre, M., Ehrlich, A.R., Gong, Z., Gilbert, Z.N., Rahiel, S., Matthews, B.J., and Vosshall, L.B. (2020). Non-canonical odor coding ensures unbreakable mosquito attraction to humans. bioRxiv, 2020.2011.2007.368720.

Zhang, Y.V., Ni, J., and Montell, C. (2013). The molecular basis for attractive salt-taste coding in Drosophila. Science 340, 1334-1338.

Zheng, Z., Lauritzen, J.S., Perlman, E., Robinson, C.G., Nichols, M., Milkie, D., Torrens, O., Price, J., Fisher, C.B., and Sharifi, N. (2018). A complete electron microscopy volume of the brain of adult Drosophila melanogaster. Cell 174, 730-743. e722. 